External Exposure Model Used in the RESRAD Code for Various Geometries of Contaminated Soil

Environmental Assessment Division Argonne National Laboratory

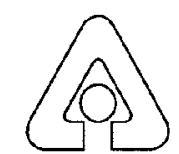

Operated by The University of Chicago, under Contract W-31-109-Eng-38, for the

United States Department of Energy 


\section{Argonne National Laboratory}

Argonne National Laboratory, with facilities in the states of Illinois and Idaho, is owned by the United States Government, and operated by the University of Chicago under the provisions of a contract with the Department of Energy.

This technical memo is a product of Argonne's Environmental Assessment Division (EAD). For information on the division's scientific and engineering activities, contact:

Director, Environmental Assessment Division

Argonne National Laboratory

Argonne, Illinois 60439-4815

Telephone (630) 252-3107

Presented in this technical memo are preliminary results of ongoing work or work that is more limited in scope and depth than that described in formal reports issued by the EAD.

Publishing support services were provided by Argonne's Information and Publishing Division (for more information, see IPD's home page: http://www.ipd.anl.gov/).

\section{Disclaimer}

This report was prepared as an account of work sponsored by an agency of the United States Government. Neither the United States Government nor any agency thereof, nor any of their employees, makes any warranty, express or implied, or assumes any legal liability or responsibility for the accuracy, completeness, or usefulness of any information, apparatus, product, or process disclosed, or represents that its use would not infringe privately owned rights. Reference herein to any specific commercial product, process, or service by trade name, trademark, manufacturer, or otherwise, does not necessarily constitute or imply its endorsement, recommendation, or favoring by the United States Government or any agency thereof. The views and opinions of authors expressed herein do not necessarily state or reflect those of the United States Government or any agency thereof.

Reproduced directly from the best available copy.

Available to DOE and DOE contractors from the Office of Scientific and Technical Information, P.O. Box 62, Oak Ridge, TN 37831; prices available from (423) 576-8401.

Available to the public from the National Technical Information Service, U.S. Department of Commerce, 5285 Port Royal Road, Springfield, VA 22161. 


\section{External Exposure Model Used in the RESRAD Code for Various Geometries of Contaminated Soil}

by S. Kamboj, C. Yu, and D.J. LePoire

Environmental Assessment Division,

Argonne National Laboratory, 9700 South Cass Avenue, Argonne, Illinois 60439

Work sponsored by United States Department of Energy, Assistant Secretary for Environment, Safety and Health, Office of Environmental Policy and Assistance, and Assistant Secretary for Environmental Management, Office of Environmental Restoration 
This report is printed on recycled paper. 


\section{CONTENTS}

ACKNOWLEDGMENTS $\ldots \ldots \ldots \ldots \ldots \ldots \ldots \ldots \ldots \ldots \ldots \ldots \ldots$ vii

NOTATION $\ldots \ldots \ldots \ldots \ldots \ldots \ldots \ldots \ldots \ldots \ldots \ldots \ldots \ldots \ldots \ldots \ldots \ldots \ldots \ldots$

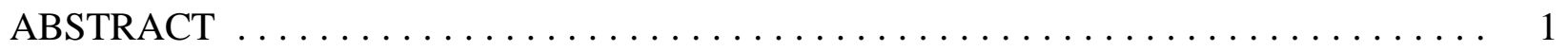

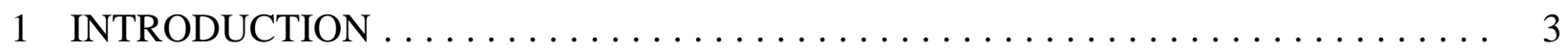

2 FGR-12 METHODOLOGY $\ldots \ldots \ldots \ldots \ldots \ldots \ldots \ldots \ldots \ldots \ldots \ldots$

3 DEPTH AND COVER-AND-DEPTH FACTORS $\ldots \ldots \ldots \ldots \ldots \ldots \ldots \ldots \ldots$

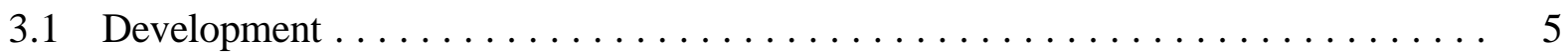

3.2 Depth Factor Comparison with FGR-12 Results $\ldots \ldots \ldots \ldots \ldots \ldots \ldots \ldots$

3.3 Cover-and-Depth Factor Comparison with FGR-12 Results $\ldots \ldots \ldots \ldots \ldots$

4 AREA AND SHAPE FACTORS $\ldots \ldots \ldots \ldots \ldots \ldots \ldots \ldots \ldots \ldots \ldots \ldots$

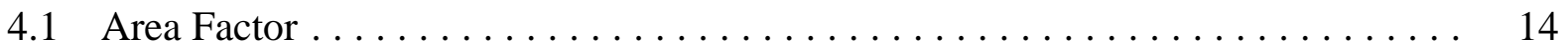

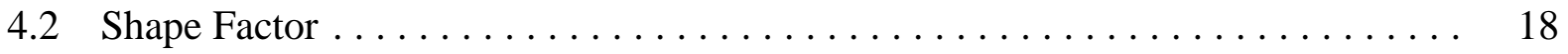

5 COMPARISON OF RESRAD MODELS $\ldots \ldots \ldots \ldots \ldots \ldots \ldots \ldots \ldots \ldots$

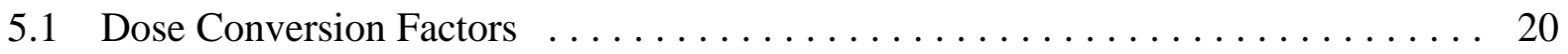

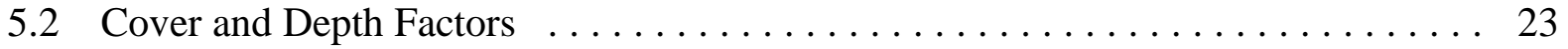

5.3 Area Factor . . . . . . . . . . . . . . . . . . . . . . . . 24

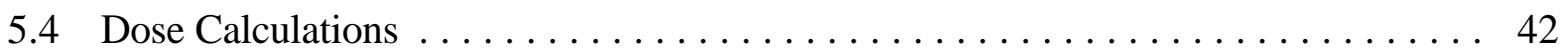

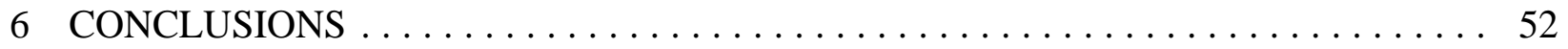

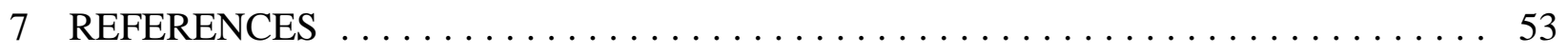

APPENDIX: External Effective Dose Equivalent Calculations for Contaminated Soil with the Monte Carlo N-Particle

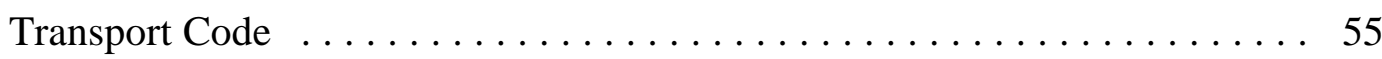

\section{TABLES}

1 Four Fitted Parameters to Calculate Cover-and-Depth

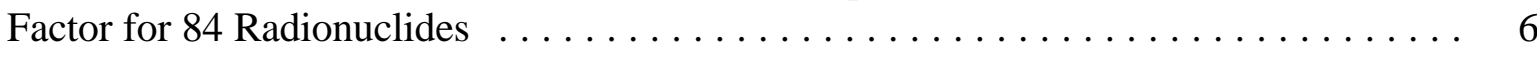




\section{TABLES (Cont.)}

2 Fitted $D C F$ s for 84 Radionuclides at 1,5 , and $15 \mathrm{~cm}$ and Effectively Infinitely Thick Sources and the Ratio of the Fitted Values to the FGR-12 Values ......... 9

3 Statistical Analysis of the Fit/FGR-12 Ratio $\ldots \ldots \ldots \ldots \ldots \ldots \ldots \ldots \ldots \ldots \ldots$

4 Comparison of Effective Dose Equivalent for Different Source

Configurations Using the Fit Parameters with FGR-12 Values . . . . . . . . . . 13

$5 \quad$ Number of Collapsed Gammas, Energies, and Their Respective

Gamma Fractions for 84 Radionuclides . . . . . . . . . . . . . . . . . . . . . . 17

6 Comparison of Infinite Thickness $D C F$ s between the

Previous RESRAD Model and FGR $-12 \ldots \ldots \ldots \ldots \ldots \ldots \ldots \ldots \ldots \ldots \ldots, 21$

7 Comparison of the Ratio of the $D C F$ s between the Previous

RESRAD Model and FGR-12 for 30-Day Equilibration Radionuclides . . . . . . . . . 24

8 Comparison of Depth Factors for Old and New RESRAD Models

for Co-60, U-234, U-235, U-238, Mn-54, Al-26, Co-57, and Cs-137 . . . . . . . . . 25

9 Depth Factor Ratio of Old to New RESRAD Models for Different Source

Depths for Co-60, U-234, U-235, U-238, Mn-54, Al-26, Co-57, and Cs-137 . . . . . 26

10 Cover-and-Depth Factor Comparison of Old and New RESRAD Models

for Various Radionuclides at Source Depths of 1,5, 15, and $50 \mathrm{~cm} \ldots \ldots \ldots \ldots .27$

11 Area Factors for Source Depths of 0.1, 1, 10, and $100 \mathrm{~cm}$ at Different Energies .... . 33

12 Area Factor Variations with Cover Thickness for Different Source Radii at an Energy of $10 \mathrm{keV}$ and Source Depths of $0.1,1,10$, and $100 \mathrm{~cm} \ldots \ldots \ldots \ldots 36$

13 Area Factor Variations with Cover Thickness for Different Source

Radii at an Energy of $100 \mathrm{keV}$ and Source Depths of 0.1, 1, 10, and $100 \mathrm{~cm}$....... 38

14 Area Factor Variations with Cover Thickness for Different Source

Radii at an Energy of $1 \mathrm{MeV}$ and Source Depths of 0.1, 1, 10, and $100 \mathrm{~cm}$........ 40

15 Dose Comparisons for Radionuclides at Source Depths of 1, 5, 15, and $50 \mathrm{~cm}$ for New and Old RESRAD Models ............................ 46

16 Dose Comparison for Co-60 as a Function of Source Depth at Different Radii for New and Old RESRAD Models . . . . . . . . . . . . . . . . . . . . 50 


\section{TABLES (Cont.)}

17 Dose Comparison for Co-60 as a Function of Cover Thickness at Different Radii for New and Old RESRAD Models $\ldots \ldots \ldots \ldots \ldots \ldots$

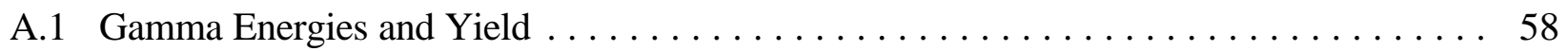

A.2 Effective Dose Equivalent per Unit Fluence for Photons Incident in Various Geometries on an Anthropomorphic Phantom . . . . . . . . . . . . 60

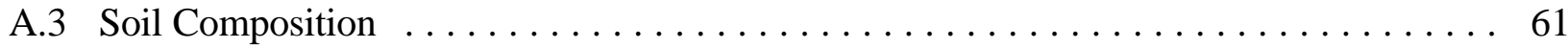

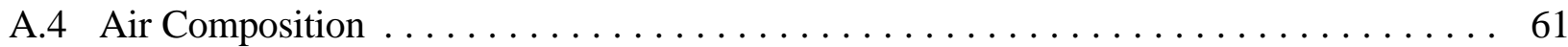

A.5 Comparison of MCNP and FGR-12 Dose Conversion

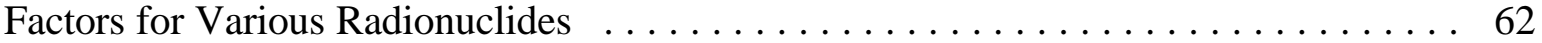

A.6 Surface Dose Comparison of MCNP and FGR-12 Values . . . . . . . . . . . . . . 62

A.7 Comparison of Dose Estimation between MCNP and the New RESRAD Model for Co-60 and Mn-54 Cylindrical Sources of Effectively Infinite Radius at Different Source Depths . . . . . . . . . . . . . 65

A.8 Comparison of Dose Calculations for MCNP and the New RESRAD Model Using the Cover-and-Depth Factor for Co-60 and Mn-54 . . . . . . . . . . . 66

A.9 Ratio of New RESRAD Model/MCNP Dose Calculations as a Function of Cover Thickness for Different Source Depths for Co-60 and Mn-54 . . . . . . . 67

\section{FIGURES}

1 Exposure Geometry Considered for Area Factor Calculation . . . . . . . . . . . . 15

2 Cross Section of Exposure Geometry Showing Element of Integration for Area Factor Calculation $\ldots \ldots \ldots \ldots \ldots \ldots \ldots \ldots \ldots \ldots$

3 Depth Factor Comparison as a Function of Depth for a Set of Radionuclides . . . . . . 26

4 Cover Factor Comparison as a Function of Cover Depth for a Set of Co-60-Contaminated Source Depths $\ldots \ldots \ldots \ldots \ldots \ldots \ldots \ldots \ldots \ldots \ldots \ldots \ldots$

5 Cover-and-Depth Factor Comparison as a Function of Cover Depth for a Set

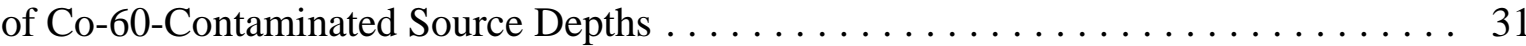




\section{FIGURES (Cont.)}

6 Area Factor Versus Source Radius for a Set of Gamma Energies with No Cover . . . . 35

7 Area Factor as a Function of Cover Thickness for a Set of Source Radii . . . . . . . . . 43

8 Dose Comparison as a Function of Source Radius for a Set of

Source Depths with No Cover $\ldots \ldots \ldots \ldots \ldots \ldots \ldots \ldots \ldots$

9 Dose Comparison of Old and New RESRAD Models as a Function of

Various Dependent Parameters for a Set of Co-60-Contaminated Source Radii . . . . . 51

A.1 Comparison of MCNP and FGR-12 Dose Values for Various Radionuclides . . . . . . 63

A.2 Comparison of FGR-12 and MCNP Dose Values for Surface Sources . . . . . . . . . 64

A.3 Dose Comparison between MCNP and New RESRAD Model as a Function

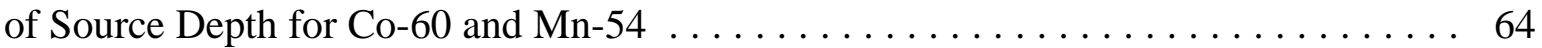

A.4 Dose Comparison between MCNP and New RESRAD Model for Co-60 and Mn-54 as a Function of Cover Thickness for Different Source Depths . . . . . . . 67

A.5 Ratio of Cover-and-Depth Factor for Co-60 and Mn-54

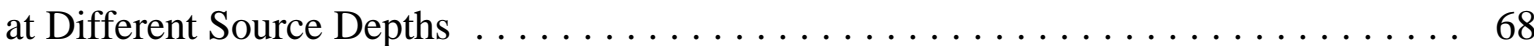




\section{ACKNOWLEDGMENTS}

The authors would like to thank Harold Peterson, Alexander Williams, and Andrew Wallo of the U.S. Department of Energy for their support of the project and review of the final report. We would also like to thank Keith Eckerman of the Oak Ridge National Laboratory and Christopher Nelson of the U.S. Environmental Protection Agency for providing comments on an earlier draft report. Finally, we thank Argonne's Information and Publishing Division, in particular John DePue of Technical Communication Services for editorial assistance and the staff of Document Production for document preparation and publishing. 


\section{NOTATION}

The following is a list of the acronyms, initialisms, and abbreviations (including units of measure) used in this document. Some acronyms used only in tables or equations are defined in the respective tables or equations.

\section{ACRONYMS, INITIALISMS, AND ABBREVIATIONS}

$\begin{array}{ll}\text { AP } & \text { anteroposterior } \\ \text { DCF } & \text { dose conversion factor } \\ \text { DOE } & \text { U.S. Department of Energy } \\ \text { EDE } & \text { effective dose equivalent } \\ \text { EPA } & \text { U.S. Environmental Protection Agency } \\ F_{A}(\text { Ei }) & \text { energy dependent area factor } \\ F_{a} \text { nuc } & \text { radionuclide specific area factor } \\ \mathrm{F}_{\mathrm{CD}} & \text { cover-and-depth factor } \\ \mathrm{F}_{\mathrm{D}} & \text { depth factor } \\ \mathrm{FGR}-12 & \text { Federal Guidance Report No. 12 } \\ \mathrm{F}_{\mathrm{S}} & \text { shape factor } \\ \text { ICRP } & \text { International Commission on Radiological Protection } \\ \text { ISO } & \text { isotropic } \\ \text { LAT } & \text { lateral } \\ \text { MCNP } & \text { Monte Carlo N-particle Transport Code } \\ \text { PA } & \text { posteroanterior } \\ \text { RESRAD } & \text { residual radioactive material guideline computer code } \\ \text { ROT } & \text { rotational }\end{array}$

\section{UNITS OF MEASURE}

$\begin{array}{llll}\mathrm{Bq} & \text { becquerel(s) } & \mathrm{m}^{2} & \text { square meter(s) } \\ \mathrm{cm} & \text { centimeter(s) } & \mathrm{m}^{3} & \begin{array}{l}\text { cubic meter(s) } \\ \mathrm{cm}^{2}\end{array} \\ \mathrm{~cm}^{3} & \text { square centimeter(s) } & \mathrm{MeV} & \text { million electron volt(s) } \\ \mathrm{g} & \text { cubic centimeter(s) } & \mathrm{mrem} & \operatorname{millirem}(\mathrm{s}) \\ \mathrm{keV} & \text { gram(s) } & \mathrm{pCi} & \operatorname{picocurie}(\mathrm{s}) \\ \mathrm{kg} & \text { kiloelectron volt(s) } & \mathrm{s} & \text { second(s) } \\ \mathrm{m} & \text { kilogram(s) } & \mathrm{Sv} & \text { sievert(s) } \\ & \text { meter(s) } & \mathrm{yr} & \text { year(s) }\end{array}$




\title{
EXTERNAL EXPOSURE MODEL USED IN THE RESRAD CODE FOR VARIOUS GEOMETRIES OF CONTAMINATED SOIL
}

by

S. Kamboj, C. Yu, and D.J. LePoire

\begin{abstract}
An external exposure model based on the U.S. Environmental Protection Agency's (EPA's) Federal Guidance Report No. 12 (FGR-12) dose conversion factors and the point kernel method has been developed for the residual radioactive (RESRAD) material guideline computer code. This model improves the external ground pathway dose estimation from that in earlier versions of the RESRAD code by extending FGR-12 data applicability to a wider range of source geometries. FGR-12 assumes that sources are infinite in lateral extent. In actual situations, soil contamination sources can have any depth, shape, cover, and size. A depth factor function was developed to express the attenuation of radionuclides by using regression analysis. Three independent, nuclei-specific parameters were determined by using the effective dose equivalent values from FGR-12. The depth factors derived with the new model were within $2 \%$ of the FGR-12 values for all depths for most of the radionuclides. A cover-and-depth factor function was derived on the basis of the depth factor function by considering both dose contribution and attenuation from different depths. The cover-and-depth factor was compared with FGR-12 computations for some representative radionuclides and source configurations. For thin cover thicknesses $(1 \mathrm{~cm})$, most of the values were within $2 \%$; even for large cover thicknesses ( 5 to $15 \mathrm{~cm}$ ), most of the values were within $10 \%$. To further extend this model for actual geometries (finite irregular areas), area and shape factors were derived by using the point kernel method. These factors depend not only on the lateral extent of the contamination but also on source depth, cover thickness, and gamma energies. The area factor increases with source radius and approaches unity for source radii greater than $50 \mathrm{~m}$. To test the integrity of FGR-12 data, effective dose equivalent values at the surface and four soil depths were compared with the Monte Carlo N-Particle (MCNP) transport code calculations for a few radionuclides. MCNP values were within $10 \%$ of the FGR values for the four soil depths. Depth and cover factors were also compared with MCNP calculations. Finally, overall comparisons were
\end{abstract}


made between the new RESRAD model (Versions 5.60 and later) and the old RESRAD model (Version 5.44 and earlier). 


\section{INTRODUCTION}

An external exposure model has been developed for Version 5.60 of the residual radioactive (RESRAD) material guideline computer code (Yu et al. 1993a). This model, which is based on the dose conversion factors from the U.S. Environmental Protection Agency's (EPA's) Federal Guidance Report No. 12 (FGR-12) (Eckerman and Ryman 1993) and the point kernel method, improves the external ground pathway dose calculations from those in earlier versions of RESRAD (Version 5.44 and earlier) (Yu et al. 1993a) and extends the applicability of FGR-12 to soil contamination of any size, shape, depth, and density.

Dose conversion factors (or coefficients) for external exposure relate the concentrations of radionuclides in environmental media to the doses to organs and tissues of the body. These dose coefficients include the energy and angular distributions of the radiations incident upon the body and the transport of these radiations within the body.

FGR-12 gives the dose coefficients for external exposure to photons and electrons emitted by radionuclides distributed in soil. The values are given for surface and uniformly distributed volume sources at four specific thicknesses $(1,5$, and $15 \mathrm{~cm}$ and effectively infinite) with a soil density of $1.6 \mathrm{~g} / \mathrm{cm}^{3}$. FGR-12 assumes that sources are infinite in lateral extent. In actual situations, sources can have any depth, shape, cover, and size. The soil density is also not fixed at $1.6 \mathrm{~g} / \mathrm{cm}^{3}$. It varies with soil type. The dry density of most soils varies within the range of 1.1 to $1.6 \mathrm{~g} / \mathrm{cm}^{3}$ (Yu et al. 1993b). A depth factor function was developed to express the attenuation of radionuclides by using regression analysis. Three independent, nuclei-specific parameters were determined by using the effective dose equivalent values of FGR-12 at different depths. A cover-and-depth factor function was derived on the basis of the depth factor function by considering both dose contribution and attenuation from different depths. To further extend this model for actual geometries (finite, irregular areas), an areaand-shape factor was derived by using the point kernel method (Kocher and Sjoreen 1985); this factor depends not only on the lateral extent of the contamination, but also on source depth, cover thickness, and gamma energies.

Section 2 describes FGR-12 methodology. Cover-and-depth factor functions are described in Section 3. Section 3 also compares depth factor and cover-and-depth factor results with FGR-12 results. Section 4 discusses area and shape factors. A comparison of RESRAD models (Version 5.44 and earlier vs. 5.60 and later) is provided in Section 5 for the following items: dose conversion factors, cover and depth factors, area factors, and dose calculations. Section 6 presents the conclusions drawn. Section 7 lists the references cited in the report, and the Appendix discusses application of the Monte Carlo N-Particle (MCNP) transport code (Briesmeister 1993), the external effective dose equivalent calculations, and the comparison of MCNP calculations with those of FGR-12 and the new RESRAD model. 


\section{FGR-12 METHODOLOGY}

The EPA publication FGR-12 (Eckerman and Ryman 1993) gives the effective dose coefficients for exposure to soil with a density of $1.6 \mathrm{~g} / \mathrm{cm}^{3}$ contaminated to thicknesses of 1,5 , and $15 \mathrm{~cm}$ and effectively infinite. The organ dose coefficients for isotropic plane sources at six source depths $(0,0.04,0.2,1.0,2.5$, and 4.0 mean free paths in soil) were integrated over source depths to compute organ dose coefficients for uniformly distributed volume sources. Dose coefficient calculations involved use of energy and angular distribution of radiation incident on the body due to monoenergetic radiation sources in contaminated soil, and transport and energy deposition of these incident particles in different organs of the body, to calculate the organ and tissue dose for the incident source and to calculate the effective dose equivalent for a specific radionuclide. The latter calculations took into account the radionuclide's energies and intensities of radiation emitted during nuclear transformation and different organ weighting factors.

Doses were first calculated for monoenergetic photon and electron sources at 12 energy levels, from 0.01 to $5.0 \mathrm{MeV}$. The results of these calculations were then used to derive the dose coefficients, taking into account the detailed nuclear decay data of each radionuclide. For organ dose calculations, a modified Cristy adult hermaphrodite phantom was used (Cristy and Eckerman 1987). The head region was modified from the original phantom to include a neck and esophagus model. This phantom represents a standing adult of $179 \mathrm{~cm}$ height and $73 \mathrm{~kg}$ mass. The weighting factors

used to calculate the effective dose equivalent were those recommended by the EPA in Radiation Protection Guidance to Federal Agencies for Occupational Exposure (EPA 1987).

The FGR-12 dose coefficients are compared with MCNP calculational results. As shown in the Appendix, the MCNP results are within 7 to $19 \%$ of the FGR-12 results. The FGR-12 dose coefficients are also compared with the dose coefficients used in the previous versions of the RESRAD code. The results are presented in Section 5. 


\section{DEPTH AND COVER-AND-DEPTH FACTORS}

\subsection{DEVELOPMENT}

The depth factor $\left(F_{D}\right)$ is based on the fits to the FGR-12 dose conversion factors $(D C F \mathrm{~s})$ as a function of depth according to the following function:

$$
F_{D}{ }^{\prime} \frac{D C F\left(T_{s}{ }^{\prime} t_{s}\right)}{D C F\left(T_{s}{ }^{\prime} 4\right)}, 1 \& A e^{\& K_{A} \mathrm{D}_{s}} \& B e^{\& K_{B} \mathrm{D}_{s}}
$$

where

$$
\begin{aligned}
D C F\left(T_{s}=t_{s}\right) & =\text { FGR-12 DCF at different depths, } \\
t_{s} & =\text { source depth }(\mathrm{cm}), \\
\mathrm{D} & =\text { soil density }\left(\mathrm{g} / \mathrm{cm}^{3}\right), \\
A, B & =\text { fit parameters }(\text { dimensionless }), \text { and } \\
K_{A}, K_{B} & =\text { fit parameters }\left(\mathrm{cm}^{2} / \mathrm{g}\right) .
\end{aligned}
$$

The following constraints were applied for the four fitting parameters:

- All the parameters are forced to be positive.

- $A+B=1$.

- In the limit source depth $t_{s} 6$ zero, the $D C F$ should be consistent with the contaminated surface $D C F$.

This method was used to determine the four unknown parameters $\left(A, B, K_{A}\right.$, and $\left.K_{B}\right)$ for 84 radionuclides available in RESRAD (Table 1). RESRAD has two radionuclide libraries, one with the cutoff half-life of 6 months (67 radionuclides) and another with the cutoff half-life of 30 days (84 radionuclides). Progeny radionuclides with a half-life less than the cutoff half-life are assumed to be in secular equilibrium with the parent radionuclide. The symbol " $+\mathrm{D}$ " is used to indicate that the short-lived decay product radionuclides are in equilibrium with the parent radionuclide and that their dose factors have been added to the parent dose factor. 
TABLE 1 Four Fitted Parameters $\left(A, B, K_{A}\right.$, and $\left.K_{B}\right)$ to Calculate Cover-and-Depth Factor for 84 Radionuclides

\begin{tabular}{|c|c|c|c|c|}
\hline Radionuclide $^{\mathrm{a}}$ & $A$ & $B$ & $K_{A}$ & $K_{B}$ \\
\hline H-3 & 0.00 & 0.00 & 0.00 & 0.00 \\
\hline C-14 & $6.421 \times 10^{-1}$ & $3.579 \times 10^{-1}$ & $2.940 \times 10^{-1}$ & 3.369 \\
\hline $\mathrm{Na}-22$ & $9.263 \times 10^{-1}$ & $7.37 \times 10^{-2}$ & $8.74 \times 10^{-2}$ & 1.331 \\
\hline Al-26 & $9.276 \times 10^{-1}$ & $7.24 \times 10^{-2}$ & $7.94 \times 10^{-2}$ & 1.284 \\
\hline S-35 & $3.405 \times 10^{-1}$ & $6.595 \times 10^{-1}$ & 3.312 & $2.846 \times 10^{-1}$ \\
\hline $\mathrm{Cl}-36$ & $8.885 \times 10^{-1}$ & $1.115 \times 10^{-1}$ & $1.325 \times 10^{-1}$ & 1.886 \\
\hline $\mathrm{K}-40$ & $7.26 \times 10^{-2}$ & $9.274 \times 10^{-1}$ & 1.269 & $7.70 \times 10^{-2}$ \\
\hline $\mathrm{Ca}-41$ & 0.00 & 0.00 & 0.00 & 0.00 \\
\hline $\mathrm{Ca}-45$ & $2.519 \times 10^{-1}$ & $7.481 \times 10^{-1}$ & 2.743 & $2.259 \times 10^{-1}$ \\
\hline Sc-46 & $7.29 \times 10^{-2}$ & $9.271 \times 10^{-1}$ & 1.352 & $8.53 \times 10^{-2}$ \\
\hline $\mathrm{Mn}-54$ & $8.48 \times 10^{-2}$ & $9.152 \times 10^{-1}$ & 1.215 & $8.79 \times 10^{-2}$ \\
\hline $\mathrm{Fe}-55$ & 0.00 & 0.00 & 0.00 & 0.00 \\
\hline $\mathrm{Fe}-59$ & $9.276 \times 10^{-1}$ & $7.24 \times 10^{-2}$ & $8.19 \times 10^{-2}$ & 1.314 \\
\hline Co-57 & $9.288 \times 10^{-1}$ & $7.12 \times 10^{-2}$ & $1.604 \times 10^{-1}$ & 1.671 \\
\hline Co-60 & $9.235 \times 10^{-1}$ & $7.65 \times 10^{-2}$ & $7.83 \times 10^{-2}$ & 1.263 \\
\hline $\mathrm{Ni}-59$ & 0.00 & 0.00 & 0.00 & 0.00 \\
\hline $\mathrm{Ni}-63$ & 0.00 & 0.00 & 0.00 & 0.00 \\
\hline $\mathrm{Zn}-65$ & $9.271 \times 10^{-1}$ & $7.29 \times 10^{-2}$ & $8.37 \times 10^{-2}$ & 1.327 \\
\hline $\mathrm{Ge}-68+\mathrm{D}$ & $9.270 \times 10^{-1}$ & $7.30 \times 10^{-2}$ & $9.94 \times 10^{-2}$ & 1.412 \\
\hline Se-75 & $6.85 \times 10^{-2}$ & $9.315 \times 10^{-1}$ & 1.552 & $1.245 \times 10^{-1}$ \\
\hline Sr- 85 & $7.210 \times 10^{-2}$ & $9.279 \times 10^{-1}$ & 1.441 & $9.99 \times 10^{-2}$ \\
\hline Sr-89 & $8.998 \times 10^{-1}$ & $1.002 \times 10^{-1}$ & $1.279 \times 10^{-1}$ & 1.763 \\
\hline Sr-90+D & $9.074 \times 10^{-1}$ & $9.260 \times 10^{-2}$ & $1.202 \times 10^{-1}$ & 1.699 \\
\hline $\mathrm{Nb}-94$ & $9.275 \times 10^{-1}$ & $7.250 \times 10^{-2}$ & $9.10 \times 10^{-2}$ & 1.378 \\
\hline $\mathrm{Nb}-95$ & $7.480 \times 10^{-2}$ & $9.252 \times 10^{-1}$ & 1.363 & $9.12 \times 10^{-2}$ \\
\hline $\mathrm{Zr}-95+\mathrm{D}$ & $9.298 \times 10^{-1}$ & $7.020 \times 10^{-2}$ & $9.30 \times 10^{-2}$ & 1.445 \\
\hline Tc-99 & $7.871 \times 10^{-1}$ & $2.129 \times 10^{-1}$ & $2.106 \times 10^{-1}$ & 2.589 \\
\hline Ru-106 & $9.271 \times 10^{-1}$ & $7.290 \times 10^{-2}$ & $9.57 \times 10^{-2}$ & 1.409 \\
\hline Ag-108m & $9.282 \times 10^{-1}$ & $7.180 \times 10^{-2}$ & $9.67 \times 10^{-2}$ & 1.442 \\
\hline $\mathrm{Ag}-110 \mathrm{~m}+\mathrm{D}$ & $9.261 \times 10^{-1}$ & $7.390 \times 10^{-2}$ & $8.74 \times 10^{-2}$ & 1.339 \\
\hline Cd-109 & $6.534 \times 10^{-1}$ & $3.466 \times 10^{-1}$ & $2.047 \times 10^{-1}$ & 4.753 \\
\hline Sn-113+D & $9.272 \times 10^{-1}$ & $7.28 \times 10^{-2}$ & $1.070 \times 10^{-1}$ & 1.652 \\
\hline $\mathrm{Sb}-124$ & $1.109 \times 10^{-1}$ & $8.891 \times 10^{-1}$ & $9.478 \times 10^{-1}$ & $7.38 \times 10^{-2}$ \\
\hline Sb-125 & $9.273 \times 10^{-1}$ & $7.270 \times 10^{-2}$ & $1.005 \times 10^{-1}$ & 1.507 \\
\hline Te-125m & $7.763 \times 10^{-1}$ & $2.237 \times 10^{-1}$ & 3.481 & $3.700 \times 10^{-1}$ \\
\hline I- 125 & $8.540 \times 10^{-1}$ & $1.460 \times 10^{-1}$ & 3.451 & $4.422 \times 10^{-1}$ \\
\hline I- 129 & $4.350 \times 10^{-1}$ & $5.650 \times 10^{-1}$ & $7.137 \times 10^{-1}$ & 3.555 \\
\hline Cs-134 & $9.266 \times 10^{-1}$ & $7.34 \times 10^{-2}$ & $9.26 \times 10^{-2}$ & 1.379 \\
\hline Cs-135 & $7.254 \times 10^{-1}$ & $2.746 \times 10^{-1}$ & $2.508 \times 10^{-1}$ & 3.030 \\
\hline Cs- $137+\mathrm{D}$ & $9.281 \times 10^{-1}$ & $7.19 \times 10^{-2}$ & $9.47 \times 10^{-2}$ & 1.411 \\
\hline $\mathrm{Ce}-141$ & $9.187 \times 10^{-1}$ & $8.13 \times 10^{-2}$ & $1.457 \times 10^{-1}$ & 1.683 \\
\hline $\mathrm{Ce}-144+\mathrm{D}$ & $9.116 \times 10^{-1}$ & $8.84 \times 10^{-2}$ & $9.38 \times 10^{-2}$ & 1.411 \\
\hline Pm-147 & $7.726 \times 10^{-1}$ & $2.274 \times 10^{-1}$ & $2.087 \times 10^{-1}$ & 2.780 \\
\hline
\end{tabular}


TABLE 1 (Cont.)

\begin{tabular}{|c|c|c|c|c|}
\hline Radionuclide $^{\mathrm{a}}$ & $A$ & $B$ & $K_{A}$ & $K_{B}$ \\
\hline Sm-147 & 0.00 & 0.00 & 0.00 & 0.00 \\
\hline Sm-151 & $3.310 \times 10^{-2}$ & $9.669 \times 10^{-1}$ & $8.270 \times 10^{-1}$ & 4.926 \\
\hline Eu-152 & $9.100 \times 10^{-1}$ & $9.000 \times 10^{-2}$ & $8.40 \times 10^{-2}$ & 1.185 \\
\hline Eu-154 & $8.939 \times 10^{-1}$ & $1.061 \times 10^{-1}$ & $8.25 \times 10^{-1}$ & 1.008 \\
\hline Eu-155 & $8.569 \times 10^{-1}$ & $1.431 \times 10^{-1}$ & $1.912 \times 10^{-1}$ & 1.486 \\
\hline Gd-152 & 0.00 & 0.00 & 0.00 & 0.00 \\
\hline Gd-153 & $8.226 \times 10^{-1}$ & $1.774 \times 10^{-1}$ & $1.986 \times 10^{-1}$ & 1.983 \\
\hline Ta-182 & $9.233 \times 10^{-1}$ & $7.670 \times 10^{-2}$ & $8.49 \times 10^{-2}$ & 1.337 \\
\hline Ir-192 & $9.306 \times 10^{-1}$ & $6.940 \times 10^{-2}$ & $1.078 \times 10^{-1}$ & 1.482 \\
\hline $\mathrm{Au}-195$ & $8.772 \times 10^{-1}$ & $1.228 \times 10^{-1}$ & $2.380 \times 10^{-1}$ & 1.880 \\
\hline Tl-204 & $8.679 \times 10^{-1}$ & $1.321 \times 10^{-1}$ & $2.068 \times 10^{-1}$ & 1.923 \\
\hline $\mathrm{Pb}-210+\mathrm{D}$ & $7.502 \times 10^{-1}$ & $2.498 \times 10^{-1}$ & $1.753 \times 10^{-1}$ & 2.200 \\
\hline Po-210 & $9.269 \times 10^{-1}$ & $7.310 \times 10^{-2}$ & $9.04 \times 10^{-2}$ & 1.385 \\
\hline $\mathrm{Bi}-207$ & $9.246 \times 10^{-1}$ & $7.540 \times 10^{-2}$ & $8.89 \times 10^{-2}$ & 1.350 \\
\hline $\mathrm{Ra}-226+\mathrm{D}$ & $9.272 \times 10^{-1}$ & $7.280 \times 10^{-2}$ & $8.35 \times 10^{-2}$ & 1.315 \\
\hline $\mathrm{Ra}-228+\mathrm{D}$ & $9.266 \times 10^{-1}$ & $7.340 \times 10^{-2}$ & $8.77 \times 10^{-2}$ & 1.371 \\
\hline Ac- $227+D$ & $9.229 \times 10^{-1}$ & $7.710 \times 10^{-2}$ & $1.172 \times 10^{-1}$ & 1.512 \\
\hline Th-228+D & $9.277 \times 10^{-1}$ & $7.230 \times 10^{-2}$ & $7.55 \times 10^{-2}$ & 1.262 \\
\hline Th-229+D & $9.130 \times 10^{-1}$ & $8.700 \times 10^{-2}$ & $1.130 \times 10^{-1}$ & 1.491 \\
\hline Th-230 & $8.628 \times 10^{-1}$ & $1.372 \times 10^{-1}$ & $1.871 \times 10^{-1}$ & 4.033 \\
\hline Th-232 & $8.152 \times 10^{-1}$ & $1.848 \times 10^{-1}$ & $2.082 \times 10^{-1}$ & 5.645 \\
\hline $\mathrm{Pa}-231$ & $9.295 \times 10^{-1}$ & $7.050 \times 10^{-2}$ & $1.163 \times 10^{-1}$ & 2.014 \\
\hline $\mathrm{U}-232$ & $8.086 \times 10^{-1}$ & $1.914 \times 10^{-1}$ & $1.754 \times 10^{-1}$ & 6.021 \\
\hline $\mathrm{U}-233$ & $8.889 \times 10^{-1}$ & $1.112 \times 10^{-1}$ & $1.394 \times 10^{-1}$ & 4.179 \\
\hline U-234 & $7.229 \times 10^{-1}$ & $2.771 \times 10^{-1}$ & $1.937 \times 10^{-1}$ & 7.238 \\
\hline U-235 & $9.292 \times 10^{-1}$ & $7.080 \times 10^{-2}$ & $1.383 \times 10^{-1}$ & 1.813 \\
\hline U-236 & $5.932 \times 10^{-1}$ & $4.068 \times 10^{-1}$ & $1.980 \times 10^{-1}$ & 8.379 \\
\hline $\mathrm{U}-238+\mathrm{D}$ & $8.590 \times 10^{-1}$ & $1.410 \times 10^{-1}$ & $9.19 \times 10^{-2}$ & 1.111 \\
\hline $\mathrm{Np}-237+\mathrm{D}$ & $9.255 \times 10^{-1}$ & $7.450 \times 10^{-2}$ & $1.228 \times 10^{-1}$ & 1.671 \\
\hline $\mathrm{Pu}-238$ & $2.972 \times 10^{-1}$ & $7.028 \times 10^{-1}$ & $1.958 \times 10^{-1}$ & 9.011 \\
\hline $\mathrm{Pu}-239$ & $8.002 \times 10^{-1}$ & $1.998 \times 10^{-1}$ & $1.348 \times 10^{-1}$ & 6.550 \\
\hline $\mathrm{Pu}-240$ & $2.977 \times 10^{-1}$ & $7.023 \times 10^{-1}$ & $2.176 \times 10^{-1}$ & 8.997 \\
\hline $\mathrm{Pu}-241$ & $9.132 \times 10^{-1}$ & $8.680 \times 10^{-2}$ & $1.582 \times 10^{-1}$ & 2.027 \\
\hline $\mathrm{Pu}-242$ & $3.314 \times 10^{-1}$ & $6.686 \times 10^{-1}$ & $2.109 \times 10^{-1}$ & 8.982 \\
\hline $\mathrm{Pu}-244$ & $9.259 \times 10^{-1}$ & $7.410 \times 10^{-2}$ & $9.26 \times 10^{-2}$ & 1.431 \\
\hline Am-241 & $8.365 \times 10^{-1}$ & $1.635 \times 10^{-1}$ & $3.130 \times 10^{-1}$ & 2.883 \\
\hline Am-243+D & $9.098 \times 10^{-1}$ & $9.020 \times 10^{-2}$ & $1.473 \times 10^{-1}$ & 1.642 \\
\hline $\mathrm{Cm}-243$ & $9.247 \times 10^{-1}$ & $7.530 \times 10^{-2}$ & $1.350 \times 10^{-1}$ & 1.662 \\
\hline $\mathrm{Cm}-244$ & $7.0 \times 10^{-3}$ & $9.930 \times 10^{-1}$ & $8.461 \times 10^{2}$ & 2.194 \\
\hline $\mathrm{Cm}-248$ & $7.333 \times 10^{-1}$ & $2.667 \times 10^{-1}$ & $1.042 \times 10^{1}$ & 1.215 \\
\hline Cf-252 & $6.505 \times 10^{-1}$ & $3.495 \times 10^{-1}$ & 7.259 & 0.182 \\
\hline
\end{tabular}

a + D means that associated decay product radionuclides with half-lives of less than 30 days are included. 
On the basis of depth factor function, the following cover-and-depth factor $\left(F_{C D}\right)$ was derived by considering both dose contribution and attenuation from different depths:

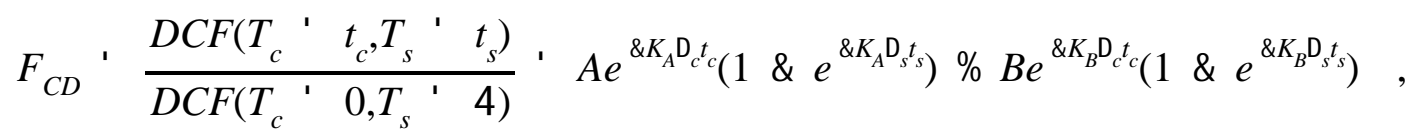

where

$$
\begin{aligned}
t_{c} & =\text { cover thickness }(\mathrm{cm}), \\
\mathrm{D}_{c} & =\text { cover density }\left(\mathrm{g} / \mathrm{cm}^{3}\right), \\
t_{s} & =\text { source depth }(\mathrm{cm}), \text { and } \\
\mathrm{D}_{s} & =\text { source density }\left(\mathrm{g} / \mathrm{cm}^{3}\right) .
\end{aligned}
$$

\subsection{DEPTH FACTOR COMPARISON WITH FGR-12 RESULTS}

Results obtained by using the fit parameters are compared with the FGR-12 data in Table 2 for 84 radionuclides for four source thicknesses. Four radionuclides (Cm-244, Cm-248, I-125, and Sm-151) showed less than $10 \%$ variation in $D C F$ between $1 \mathrm{~cm}$ and infinite depth values. Among these four, Sm-151 showed the smallest variation (1\%). The overall comparison shows that most of the fit data are within 3\% of the FGR-12 data, except for eight points. For these eight points, fit values are mostly higher than FGR data (two are 5\% higher, one is $3.2 \%$ lower, and five are $4 \%$ higher). A statistical analysis of the comparison is summarized in Table 3.

\subsection{COVER-AND-DEPTH FACTOR COMPARISON WITH FGR-12 RESULTS}

The results of dose calculations using the cover-and-depth factor for a few geometries are compared with FGR-12 radionuclide-specific dose calculations in Table 4. The comparisons were done for cadmium-109, cesium-137, cobalt-60, manganese-54, and aluminum-26. For the FGR-12 calculations, the effective dose equivalent for a source thickness of $4 \mathrm{~cm}$ with $1 \mathrm{~cm}$ cover, for

example, was obtained by subtracting the value for a 1-cm-thick source from the value for a 5-cm-thick source. For a cover thickness of $1 \mathrm{~cm}$, most values from RESRAD calculations were within $2 \%$ of the FGR-12 values, and the maximum difference was $5 \%$. For cover thicknesses of $5 \mathrm{~cm}$ and $15 \mathrm{~cm}$, most values were within $10 \%$, and the maximum difference was less than $20 \%$. 
TABLE 2 Fitted $D C F$ s for 84 Radionuclides at 1, 5, and $15 \mathrm{~cm}$ and Effectively Infinitely Thick Sources and the Ratio of the Fitted Values to the FGR-12 Values

\begin{tabular}{|c|c|c|c|c|c|c|c|c|}
\hline \multirow[b]{2}{*}{ Radionuclide $^{\mathrm{a}}$} & \multicolumn{4}{|c|}{ Fitted DCF $[(\mathrm{mrem} / \mathrm{yr}) /(\mathrm{pCi} / \mathrm{g})]$} & \multicolumn{4}{|c|}{ Fit/FGR-12 Ratio } \\
\hline & $1 \mathrm{~cm}$ & $5 \mathrm{~cm}$ & $15 \mathrm{~cm}$ & Infinite & $1 \mathrm{~cm}$ & $5 \mathrm{~cm}$ & $15 \mathrm{~cm}$ & Infinite \\
\hline Ac-227+D & $4.60 \times 10^{-1}$ & 1.29 & 1.91 & 2.02 & 1.00 & 1.00 & 1.01 & 1.00 \\
\hline Ag-108m+D & 1.92 & 5.54 & 8.80 & 9.67 & 1.00 & 1.00 & 1.02 & 1.00 \\
\hline $\mathrm{Ag}-110 \mathrm{~m}+\mathrm{D}$ & 3.19 & 9.26 & $1.52 \times 10^{1}$ & $1.72 \times 10^{1}$ & $9.97 \times 10^{-1}$ & $9.97 \times 10^{-1}$ & 1.03 & 1.00 \\
\hline Al-26 & 3.01 & 8.81 & $1.50 \times 10^{1}$ & $1.74 \times 10^{1}$ & $9.89 \times 10^{-1}$ & $9.93 \times 10^{-1}$ & 1.03 & $9.95 \times 10^{-1}$ \\
\hline Am-241 & $2.15 \times 10^{-2}$ & $4.08 \times 10^{-2}$ & $4.38 \times 10^{-2}$ & $4.38 \times 10^{-2}$ & 1.00 & 1.00 & $9.99 \times 10^{-1}$ & 1.00 \\
\hline Am-243+D & $2.46 \times 10^{-1}$ & $6.45 \times 10^{-1}$ & $8.73 \times 10^{-1}$ & $8.97 \times 10^{-1}$ & $9.99 \times 10^{-1}$ & $9.99 \times 10^{-1}$ & 1.00 & 1.00 \\
\hline $\mathrm{Au}-195$ & $8.21 \times 10^{-2}$ & $1.81 \times 10^{-1}$ & $2.07 \times 10^{-1}$ & $2.08 \times 10^{-1}$ & 1.00 & 1.00 & $9.98 \times 10^{-1}$ & 1.00 \\
\hline Bi-207 & 1.78 & 5.13 & 8.37 & 9.40 & 1.00 & 1.00 & 1.03 & 1.00 \\
\hline C-14 & $8.06 \times 10^{-6}$ & $1.27 \times 10^{-5}$ & $1.35 \times 10^{-5}$ & $1.35 \times 10^{-5}$ & 1.00 & 1.00 & 1.00 & 1.00 \\
\hline $\mathrm{Ca}-41$ & 0.00 & 0.00 & 0.00 & 0.00 & - & - & - & - \\
\hline $\mathrm{Ca}-45$ & $2.98 \times 10^{-5}$ & $5.49 \times 10^{-5}$ & $6.24 \times 10^{-5}$ & $6.26 \times 10^{-5}$ & 1.00 & 1.00 & $9.97 \times 10^{-1}$ & 1.00 \\
\hline Cd-109 & $7.78 \times 10^{-3}$ & $1.28 \times 10^{-2}$ & $1.46 \times 10^{-2}$ & $1.47 \times 10^{-2}$ & $9.99 \times 10^{-1}$ & $9.98 \times 10^{-1}$ & $9.92 \times 10^{-1}$ & $9.98 \times 10^{-1}$ \\
\hline $\mathrm{Ce}-141$ & $8.49 \times 10^{-2}$ & $2.27 \times 10^{-1}$ & $3.09 \times 10^{-1}$ & $3.18 \times 10^{-1}$ & $9.99 \times 10^{-1}$ & 1.00 & $9.86 \times 10^{-1}$ & $9.99 \times 10^{-1}$ \\
\hline $\mathrm{Ce}-144+\mathrm{D}$ & $6.69 \times 10^{-2}$ & $1.85 \times 10^{-1}$ & $2.94 \times 10^{-1}$ & $3.25 \times 10^{-1}$ & $9.99 \times 10^{-1}$ & 1.00 & 1.04 & 1.00 \\
\hline Cf- 252 & $1.30 \times 10^{-4}$ & $1.62 \times 10^{-4}$ & $1.75 \times 10^{-4}$ & $1.76 \times 10^{-4}$ & $9.99 \times 10^{-1}$ & 1.00 & $9.95 \times 10^{-1}$ & 1.00 \\
\hline $\mathrm{Cl}-36$ & $6.62 \times 10^{-4}$ & $1.66 \times 10^{-3}$ & $2.31 \times 10^{-3}$ & $2.40 \times 10^{-3}$ & 1.00 & 1.00 & 1.01 & 1.00 \\
\hline $\mathrm{Cm}-243$ & $1.46 \times 10^{-1}$ & $4.01 \times 10^{-1}$ & $5.63 \times 10^{-1}$ & $5.84 \times 10^{-1}$ & $9.99 \times 10^{-1}$ & 1.00 & $9.96 \times 10^{-1}$ & 1.00 \\
\hline $\mathrm{Cm}-244$ & $1.22 \times 10^{-4}$ & $1.26 \times 10^{-4}$ & $1.26 \times 10^{-4}$ & $1.26 \times 10^{-4}$ & $9.98 \times 10^{-1}$ & $9.98 \times 10^{-1}$ & $9.98 \times 10^{-1}$ & $9.98 \times 10^{-1}$ \\
\hline $\mathrm{Cm}-248$ & $8.47 \times 10^{-5}$ & $8.80 \times 10^{-5}$ & $8.80 \times 10^{-5}$ & $8.80 \times 10^{-5}$ & 1.00 & 1.00 & 1.00 & 1.00 \\
\hline Co-57 & $1.39 \times 10^{-1}$ & $3.72 \times 10^{-1}$ & $4.92 \times 10^{-1}$ & $5.02 \times 10^{-1}$ & $9.98 \times 10^{-1}$ & $9.99 \times 10^{-1}$ & $9.88 \times 10^{-1}$ & 1.00 \\
\hline Co-60 & 2.82 & 8.18 & $1.39 \times 10^{1}$ & $1.62 \times 10^{1}$ & $9.92 \times 10^{-1}$ & $9.82 \times 10^{-1}$ & 1.02 & $9.96 \times 10^{-1}$ \\
\hline Cs-134 & 1.83 & 5.31 & 8.55 & 9.49 & 1.00 & 1.00 & 1.02 & 1.00 \\
\hline Cs-135 & $1.97 \times 10^{-5}$ & $3.47 \times 10^{-5}$ & $3.83 \times 10^{-5}$ & $3.84 \times 10^{-5}$ & 1.00 & 1.00 & $9.98 \times 10^{-1}$ & 1.00 \\
\hline Cs $-137+\mathrm{D}$ & $6.68 \times 10^{-1}$ & 1.94 & 3.10 & 3.42 & 1.00 & 1.00 & 1.02 & 1.00 \\
\hline $\mathrm{Eu}-152$ & 1.34 & 3.76 & 6.17 & 7.02 & 1.02 & $9.89 \times 10^{-1}$ & 1.02 & 1.00 \\
\hline Eu-154 & 1.50 & 4.12 & 6.73 & 7.68 & 1.05 & $9.96 \times 10^{-1}$ & 1.02 & $9.99 \times 10^{-1}$ \\
\hline Eu-155 & $6.51 \times 10^{-2}$ & $1.49 \times 10^{-1}$ & $1.81 \times 10^{-1}$ & $1.83 \times 10^{-1}$ & 1.03 & 1.00 & $9.94 \times 10^{-1}$ & 1.00 \\
\hline $\mathrm{Fe}-55$ & 0.00 & 0.00 & 0.00 & 0.00 & - & - & - & - \\
\hline $\mathrm{Fe}-59$ & 1.35 & 3.96 & 6.65 & 7.63 & $9.98 \times 10^{-1}$ & 1.00 & 1.03 & $9.99 \times 10^{-1}$ \\
\hline
\end{tabular}


TABLE 2 (Cont.)

\begin{tabular}{|c|c|c|c|c|c|c|c|c|}
\hline \multirow[b]{2}{*}{ Radionuclide $^{\mathrm{a}}$} & \multicolumn{4}{|c|}{ Fitted DCF $[(\mathrm{mrem} / \mathrm{yr}) /(\mathrm{pCi} / \mathrm{g})]$} & \multicolumn{4}{|c|}{ Fit/FGR-12 Ratio } \\
\hline & $1 \mathrm{~cm}$ & $5 \mathrm{~cm}$ & $15 \mathrm{~cm}$ & Infinite & $1 \mathrm{~cm}$ & $5 \mathrm{~cm}$ & $15 \mathrm{~cm}$ & Infinite \\
\hline Gd-152 & 0.00 & 0.00 & 0.00 & 0.00 & - & - & - & - \\
\hline Gd-153 & $9.65 \times 10^{-2}$ & $2.04 \times 10^{-1}$ & $2.43 \times 10^{-1}$ & $2.45 \times 10^{-1}$ & $9.99 \times 10^{-1}$ & 1.00 & $9.92 \times 10^{-1}$ & $9.99 \times 10^{-1}$ \\
\hline Ge-68+D & 1.13 & 3.27 & 5.15 & 5.63 & $9.96 \times 10^{-1}$ & $9.97 \times 10^{-1}$ & 1.01 & $9.99 \times 10^{-1}$ \\
\hline $\mathrm{H}-3$ & 0.00 & 0.00 & 0.00 & 0.00 & - & - & - & - \\
\hline $\mathrm{I}-125$ & $1.54 \times 10^{-2}$ & $1.65 \times 10^{-2}$ & $1.66 \times 10^{-2}$ & $1.66 \times 10^{-2}$ & 1.00 & $9.98 \times 10^{-1}$ & 1.00 & 1.00 \\
\hline $\mathrm{I}-129$ & $1.12 \times 10^{-2}$ & $1.30 \times 10^{-2}$ & $1.30 \times 10^{-2}$ & $1.30 \times 10^{-2}$ & 1.00 & 1.00 & 1.00 & 1.00 \\
\hline Ir-192 & $9.72 \times 10^{-1}$ & 2.81 & 4.30 & 4.62 & 1.00 & 1.00 & 1.01 & 1.00 \\
\hline $\mathrm{K}-40$ & $1.78 \times 10^{-1}$ & $5.19 \times 10^{-1}$ & $8.88 \times 10^{-1}$ & 1.04 & $9.99 \times 10^{-1}$ & $9.98 \times 10^{-1}$ & 1.04 & $9.96 \times 10^{-1}$ \\
\hline Mn-54 & $9.98 \times 10^{-1}$ & 2.83 & 4.60 & 5.17 & 1.02 & 1.00 & 1.02 & 1.00 \\
\hline $\mathrm{Na}-22$ & 2.54 & 7.38 & $1.21 \times 10^{1}$ & $1.37 \times 10^{1}$ & $9.99 \times 10^{-1}$ & $9.97 \times 10^{-1}$ & 1.03 & 1.00 \\
\hline $\mathrm{Nb}-94$ & 1.84 & 5.35 & 8.69 & 9.70 & $9.98 \times 10^{-1}$ & 1.00 & 1.02 & 1.00 \\
\hline $\mathrm{Nb}-95$ & $9.00 \times 10^{-1}$ & 2.60 & 4.20 & 4.69 & 1.00 & $9.99 \times 10^{-1}$ & 1.02 & 1.00 \\
\hline Ni-59 & 0.00 & 0.00 & 0.00 & 0.00 & - & - & - & - \\
\hline Ni-63 & 0.00 & 0.00 & 0.00 & 0.00 & - & - & - & - \\
\hline $\mathrm{Np}-237+\mathrm{D}$ & $2.58 \times 10^{-1}$ & $7.19 \times 10^{-1}$ & 1.05 & 1.10 & $9.99 \times 10^{-1}$ & 1.00 & 1.00 & 1.00 \\
\hline $\mathrm{Pa}-231$ & $4.29 \times 10^{-2}$ & $1.21 \times 10^{-1}$ & $1.80 \times 10^{-1}$ & $1.91 \times 10^{-1}$ & $9.97 \times 10^{-1}$ & $9.99 \times 10^{-1}$ & 1.00 & 1.00 \\
\hline $\mathrm{Pb}-210+\mathrm{D}$ & $2.58 \times 10^{-3}$ & $4.93 \times 10^{-3}$ & $5.98 \times 10^{-3}$ & $6.05 \times 10^{-3}$ & 1.00 & $9.88 \times 10^{-1}$ & 1.01 & 1.00 \\
\hline Pm-147 & $2.23 \times 10^{-5}$ & $4.29 \times 10^{-5}$ & $4.99 \times 10^{-5}$ & $5.02 \times 10^{-5}$ & 1.00 & 1.00 & $9.99 \times 10^{-1}$ & 1.00 \\
\hline Po-210 & $9.90 \times 10^{-6}$ & $2.87 \times 10^{-5}$ & $4.67 \times 10^{-5}$ & $5.23 \times 10^{-5}$ & $9.96 \times 10^{-1}$ & $9.97 \times 10^{-1}$ & 1.02 & 1.00 \\
\hline $\mathrm{Pu}-238$ & $1.19 \times 10^{-4}$ & $1.43 \times 10^{-4}$ & $1.52 \times 10^{-4}$ & $1.52 \times 10^{-4}$ & 1.00 & 1.00 & 1.00 & 1.00 \\
\hline $\mathrm{Pu}-239$ & $1.05 \times 10^{-4}$ & $2.16 \times 10^{-4}$ & $2.87 \times 10^{-4}$ & $2.96 \times 10^{-4}$ & 1.00 & $9.98 \times 10^{-1}$ & 1.01 & 1.00 \\
\hline $\mathrm{Pu}-240$ & $1.16 \times 10^{-4}$ & $1.39 \times 10^{-4}$ & $1.47 \times 10^{-4}$ & $1.47 \times 10^{-4}$ & 1.00 & 1.00 & 1.00 & 1.00 \\
\hline $\mathrm{Pu}-241+\mathrm{D}$ & $5.44 \times 10^{-6}$ & $1.40 \times 10^{-5}$ & $1.85 \times 10^{-5}$ & $1.89 \times 10^{-5}$ & $9.99 \times 10^{-1}$ & $9.97 \times 10^{-1}$ & $9.89 \times 10^{-1}$ & $9.98 \times 10^{-1}$ \\
\hline $\mathrm{Pu}-242$ & $9.78 \times 10^{-5}$ & $1.20 \times 10^{-4}$ & $1.28 \times 10^{-4}$ & $1.28 \times 10^{-4}$ & $9.98 \times 10^{-1}$ & $9.99 \times 10^{-1}$ & $9.96 \times 10^{-1}$ & $9.98 \times 10^{-1}$ \\
\hline $\mathrm{Pu}-244+\mathrm{D}$ & 1.50 & 4.32 & 6.94 & 7.73 & $9.94 \times 10^{-1}$ & $9.93 \times 10^{-1}$ & 1.02 & 1.00 \\
\hline Ra-226+D & 2.01 & 5.85 & 9.78 & $1.12 \times 10^{1}$ & $9.97 \times 10^{-1}$ & $9.96 \times 10^{-1}$ & 1.03 & $9.98 \times 10^{-1}$ \\
\hline Ra-228+D & 1.12 & 3.24 & 5.32 & 5.99 & 1.00 & 1.00 & 1.03 & $9.99 \times 10^{-1}$ \\
\hline Ru-106+D & $2.55 \times 10^{-1}$ & $7.35 \times 10^{-1}$ & 1.17 & 1.29 & 1.00 & $9.99 \times 10^{-1}$ & 1.02 & $9.97 \times 10^{-1}$ \\
\hline S-35 & $8.64 \times 10^{-6}$ & $1.39 \times 10^{-5}$ & $1.49 \times 10^{-5}$ & $1.49 \times 10^{-5}$ & 1.00 & 1.00 & 1.00 & 1.00 \\
\hline Sb-124 & 2.18 & 5.95 & 9.94 & $1.17 \times 10^{1}$ & 1.05 & $9.82 \times 10^{-1}$ & 1.01 & 1.00 \\
\hline
\end{tabular}


TABLE 2 (Cont.)

\begin{tabular}{|c|c|c|c|c|c|c|c|c|}
\hline \multirow[b]{2}{*}{ Radionuclide $^{\mathrm{a}}$} & \multicolumn{4}{|c|}{ Fitted DCF $[(\mathrm{mrem} / \mathrm{yr}) /(\mathrm{pCi} / \mathrm{g})]$} & \multicolumn{4}{|c|}{ Fit/FGR-12 Ratio } \\
\hline & $1 \mathrm{~cm}$ & $5 \mathrm{~cm}$ & $15 \mathrm{~cm}$ & Infinite & $1 \mathrm{~cm}$ & $5 \mathrm{~cm}$ & $15 \mathrm{~cm}$ & Infinite \\
\hline $\mathrm{Sb}-125$ & $4.99 \times 10^{-1}$ & 1.43 & 2.24 & 2.45 & $9.99 \times 10^{-1}$ & $9.99 \times 10^{-1}$ & 1.02 & 1.00 \\
\hline Sc-46 & 2.32 & 6.74 & $1.12 \times 10^{1}$ & $1.27 \times 10^{1}$ & $9.99 \times 10^{-1}$ & $9.99 \times 10^{-1}$ & 1.03 & 1.00 \\
\hline $\mathrm{Se}-75$ & $4.57 \times 10^{-1}$ & 1.30 & 1.89 & 1.98 & $9.98 \times 10^{-1}$ & $9.98 \times 10^{-1}$ & $9.99 \times 10^{-1}$ & 1.00 \\
\hline Sm-147 & 0.00 & 0.00 & 0.00 & 0.00 & - & - & - & - \\
\hline Sm-151 & $9.78 \times 10^{-7}$ & $9.87 \times 10^{-7}$ & $9.87 \times 10^{-7}$ & $9.87 \times 10^{-7}$ & 1.00 & 1.00 & 1.00 & 1.00 \\
\hline Sn-113+D & $3.12 \times 10^{-1}$ & $8.85 \times 10^{-1}$ & 1.36 & 1.46 & $9.97 \times 10^{-1}$ & $9.97 \times 10^{-1}$ & 1.00 & $9.97 \times 10^{-1}$ \\
\hline Sr-85 & $6.00 \times 10^{-1}$ & 1.73 & 2.72 & 2.97 & 1.00 & 1.00 & 1.01 & 1.00 \\
\hline Sr-89 & $2.37 \times 10^{-3}$ & $6.15 \times 10^{-3}$ & $8.70 \times 10^{-3}$ & $9.08 \times 10^{-3}$ & 1.00 & 1.00 & 1.01 & 1.00 \\
\hline Sr-90+D & $6.06 \times 10^{-3}$ & $1.61 \times 10^{-2}$ & $2.34 \times 10^{-2}$ & $2.47 \times 10^{-2}$ & 1.00 & 1.00 & 1.01 & 1.00 \\
\hline Ta-182 & 1.47 & 4.23 & 6.99 & 7.93 & 1.00 & 1.00 & 1.04 & $9.99 \times 10^{-1}$ \\
\hline Tc-99 & $5.48 \times 10^{-5}$ & $1.08 \times 10^{-4}$ & $1.25 \times 10^{-4}$ & $1.26 \times 10^{-4}$ & 1.00 & 1.00 & 1.00 & 1.00 \\
\hline $\mathrm{Te}-125 \mathrm{~m}$ & $1.33 \times 10^{-2}$ & $1.50 \times 10^{-2}$ & $1.52 \times 10^{-2}$ & $1.52 \times 10^{-2}$ & 1.00 & 1.01 & 1.00 & 1.00 \\
\hline Th-228+D & 1.71 & 5.00 & 8.62 & $1.02 \times 10^{1}$ & $9.91 \times 10^{-1}$ & $9.92 \times 10^{-1}$ & 1.04 & $9.94 \times 10^{-1}$ \\
\hline Th-229+D & $3.63 \times 10^{-1}$ & $9.96 \times 10^{-1}$ & 1.48 & 1.58 & 1.00 & 1.00 & 1.02 & $9.99 \times 10^{-1}$ \\
\hline Th-230 & $4.36 \times 10^{-4}$ & $9.76 \times 10^{-4}$ & $1.20 \times 10^{-3}$ & $1.21 \times 10^{-3}$ & $9.98 \times 10^{-1}$ & $9.99 \times 10^{-1}$ & 1.00 & $9.99 \times 10^{-1}$ \\
\hline Th-232 & $2.17 \times 10^{-4}$ & $4.41 \times 10^{-4}$ & $5.19 \times 10^{-4}$ & $5.22 \times 10^{-4}$ & $9.99 \times 10^{-1}$ & $9.99 \times 10^{-1}$ & $9.97 \times 10^{-1}$ & 1.00 \\
\hline Tl-204 & $1.51 \times 10^{-3}$ & $3.39 \times 10^{-3}$ & $4.04 \times 10^{-3}$ & $4.06 \times 10^{-3}$ & 1.00 & $9.99 \times 10^{-1}$ & 1.00 & $9.99 \times 10^{-1}$ \\
\hline $\mathrm{U}-232$ & $3.51 \times 10^{-4}$ & $7.24 \times 10^{-4}$ & $8.93 \times 10^{-4}$ & $9.04 \times 10^{-4}$ & $9.98 \times 10^{-1}$ & $9.99 \times 10^{-1}$ & 1.00 & 1.00 \\
\hline U-233 & $4.03 \times 10^{-4}$ & $9.91 \times 10^{-4}$ & $1.36 \times 10^{-3}$ & $1.40 \times 10^{-3}$ & $9.98 \times 10^{-1}$ & $9.99 \times 10^{-1}$ & 1.00 & 1.00 \\
\hline $\mathrm{U}-234$ & $1.89 \times 10^{-4}$ & $3.41 \times 10^{-4}$ & $4.00 \times 10^{-4}$ & $4.03 \times 10^{-4}$ & 1.00 & 1.00 & 1.00 & 1.00 \\
\hline $\mathrm{U}-235+\mathrm{D}$ & $1.91 \times 10^{-1}$ & $5.25 \times 10^{-1}$ & $7.33 \times 10^{-1}$ & $7.59 \times 10^{-1}$ & $9.99 \times 10^{-1}$ & $9.99 \times 10^{-1}$ & $9.93 \times 10^{-1}$ & 1.00 \\
\hline U-236 & $1.22 \times 10^{-4}$ & $1.89 \times 10^{-4}$ & $2.14 \times 10^{-4}$ & $2.15 \times 10^{-4}$ & $9.99 \times 10^{-1}$ & 1.00 & 1.01 & $9.98 \times 10^{-1}$ \\
\hline U-238+D & $3.08 \times 10^{-2}$ & $8.32 \times 10^{-2}$ & $1.23 \times 10^{-1}$ & $1.37 \times 10^{-1}$ & 1.04 & $9.68 \times 10^{-1}$ & 1.01 & 1.00 \\
\hline $\mathrm{Zn}-65$ & $6.71 \times 10^{-1}$ & 1.95 & 3.25 & 3.71 & 1.00 & 1.00 & 1.03 & 1.00 \\
\hline $\mathrm{Zr}-95+\mathrm{D}$ & $8.67 \times 10^{-1}$ & 2.52 & 4.07 & 4.52 & $9.99 \times 10^{-1}$ & $9.99 \times 10^{-1}$ & 1.02 & $9.99 \times 10^{-1}$ \\
\hline
\end{tabular}

a + D means that associated decay product radionuclides with half-lives less than 30 days are included. 
TABLE 3 Statistical Analysis of the Fit/FGR-12 Ratio

\begin{tabular}{|c|c|c|c|c|}
\hline \multirow[b]{2}{*}{ Statistical Parameter } & \multicolumn{4}{|c|}{ Fit/FGR-12 Ratio, by Source Thickness } \\
\hline & $1 \mathrm{~cm}$ & $5 \mathrm{~cm}$ & $15 \mathrm{~cm}$ & Infinite \\
\hline Number of data points ${ }^{\mathrm{a}}$ & 77 & 77 & 77 & 77 \\
\hline Average & 1.00 & 0.998 & 1.01 & 0.999 \\
\hline Standard deviation & 0.011 & 0.005 & 0.014 & 0.001 \\
\hline Maximum deviation & $5 \%(\mathrm{Eu}-155, \mathrm{Sb}-124)$ & $4 \%(\mathrm{U}-238)$ & $\begin{array}{c}4 \%(\mathrm{Ce}-144, \mathrm{~K}-40 \\
\text { Ta-182, Th-228) }\end{array}$ & $1 \%$ \\
\hline
\end{tabular}

a Statistical analysis was done only for radionuclides with nonzero $D C F s$ (the $D C F$ s for $\mathrm{Ca}-41, \mathrm{Fe}$ 55, Gd-152, H-3, Ni-59, Ni-63, and Sm-147 were zero). 
TABLE 4 Comparison of Effective Dose Equivalent for Different Source Configurations Using the Fit Parameters with FGR-12 Values ${ }^{\mathrm{a}}$

\begin{tabular}{|c|c|c|c|c|c|c|c|c|c|c|}
\hline \multirow[b]{3}{*}{ Source Configurations } & \multicolumn{10}{|c|}{ Effective Dose Equivalent [(mrem/yr)/(pCi/g)] } \\
\hline & \multicolumn{2}{|c|}{ Cd-109 } & \multicolumn{2}{|c|}{ Cs-137 } & \multicolumn{2}{|c|}{ Mn-54 } & \multicolumn{2}{|c|}{ Co-60 } & \multicolumn{2}{|c|}{ Al-26 } \\
\hline & Fit & FGR-12 & Fit & FGR-12 & Fit & FGR-12 & Fit & FGR-12 & Fit & FGR-12 \\
\hline Cover $=1 \mathrm{~cm}$, source $=4 \mathrm{~cm}$ & 0.0051 & 0.0051 & 1.26 & 1.26 & 1.83 & 1.85 & 5.37 & 5.47 & 5.83 & 5.83 \\
\hline Cover $=1 \mathrm{~cm}$, source $=14 \mathrm{~cm}$ & 0.0069 & 0.0069 & 2.42 & 2.36 & 3.59 & 3.51 & 11.1 & 10.7 & 12.0 & 11.4 \\
\hline Cover $=1 \mathrm{~cm}$, source $=$ infinite & 0.0069 & 0.0069 & 2.75 & 2.75 & 4.16 & 4.18 & 13.4 & 13.4 & 14.4 & 14.4 \\
\hline Cover $=5 \mathrm{~cm}$, source $=10 \mathrm{~cm}$ & 0.0018 & 0.0019 & 1.16 & 1.10 & 1.76 & 1.66 & 5.72 & 5.23 & 6.15 & 5.59 \\
\hline Cover $=5 \mathrm{~cm}$, source $=$ infinite & 0.0019 & 0.0019 & 1.48 & 1.48 & 2.34 & 2.34 & 8.00 & 7.90 & 8.56 & 8.56 \\
\hline Cover $=15 \mathrm{~cm}$, source $=$ infinite & 0.000 & 0.000 & 0.33 & 0.39 & 0.57 & 0.67 & 2.29 & 2.67 & 2.40 & 2.97 \\
\hline
\end{tabular}

${ }^{\text {a }}$ See Section 3.3 for calculation of the FGR-12 effective dose equivalents. 


\section{AREA AND SHAPE FACTORS}

\subsection{AREA FACTOR}

The energy-dependent area factor, $F_{A}(E i)$, can be derived by considering the point kernel dose integral, $D\left(R, t_{a}, t_{c}, t_{s}\right)$, over the source thickness $\left(t_{s}\right)$, radius $(R)$, distance from the receptor to the plane of the source and air interface $\left(t_{a}\right)$, and thickness of the shielding material $\left(t_{c}\right)$ for the rotational (ROT) geometry depicted in Figure 1. The area factor is the ratio of the dose integrals for the geometry being considered and the infinite slab geometry:

$$
\left.F_{A}(E i)\right)^{\prime} \frac{D\left(R^{\prime} r, T_{a}^{\prime} 1 \mathrm{~m}, T_{c}{ }^{\prime} t_{c}, T_{s}{ }^{\prime} t_{s}\right)}{D\left(R^{\prime} 4, T_{a}{ }^{\prime} 1 \mathrm{~m}, T_{c}{ }^{\prime} t_{c}, T_{s}{ }^{\prime} t_{s}\right)}
$$

where the function $D$ is the dose evaluated by using the point kernel method (Figure 2):

$$
D\left(R, t_{a}, t_{c}, t_{s}\right)^{\prime} \quad K \operatorname{m}_{V_{s}} e^{\&} \frac{B(z)}{4 \mathrm{~B} l^{2}} d V_{S}
$$

where

$$
\begin{aligned}
& z=\frac{\mu_{a} t_{a} \% \mu_{c} t_{c} \% \mu_{s} t}{t_{a} \% t_{c} \% t} l \\
& l^{2}=r^{2} \%\left(t_{a} \% t_{c} \% t\right)^{2} ; \\
& d V_{S}=2 \mathrm{~B} r t d r d t \\
& \mu_{a}=\text { attenuation coefficient for air }\left(\mathrm{cm}^{-1}\right) \text {; } \\
& \mu_{c}=\text { attenuation coefficient for the cover material }\left(\mathrm{cm}^{-1}\right) \text {; } \\
& \mu_{s}=\text { attenuation coefficient of the source material }\left(\mathrm{cm}^{-1}\right) \text {; } \\
& B(z)=\text { buildup factor (G-P Method [Trubey 1991]) for length measured in } \\
& \text { mean free paths, } z \text {; and } \\
& K=\text { energy-dependent conversion factor. }
\end{aligned}
$$




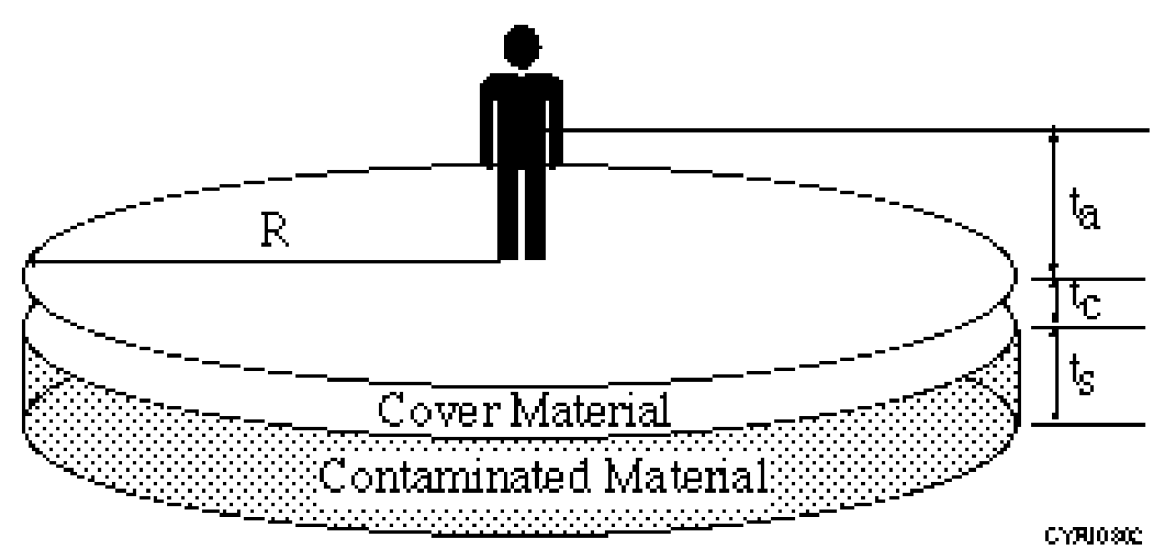

FIGURE 1 Exposure Geometry Considered for Area Factor Calculation

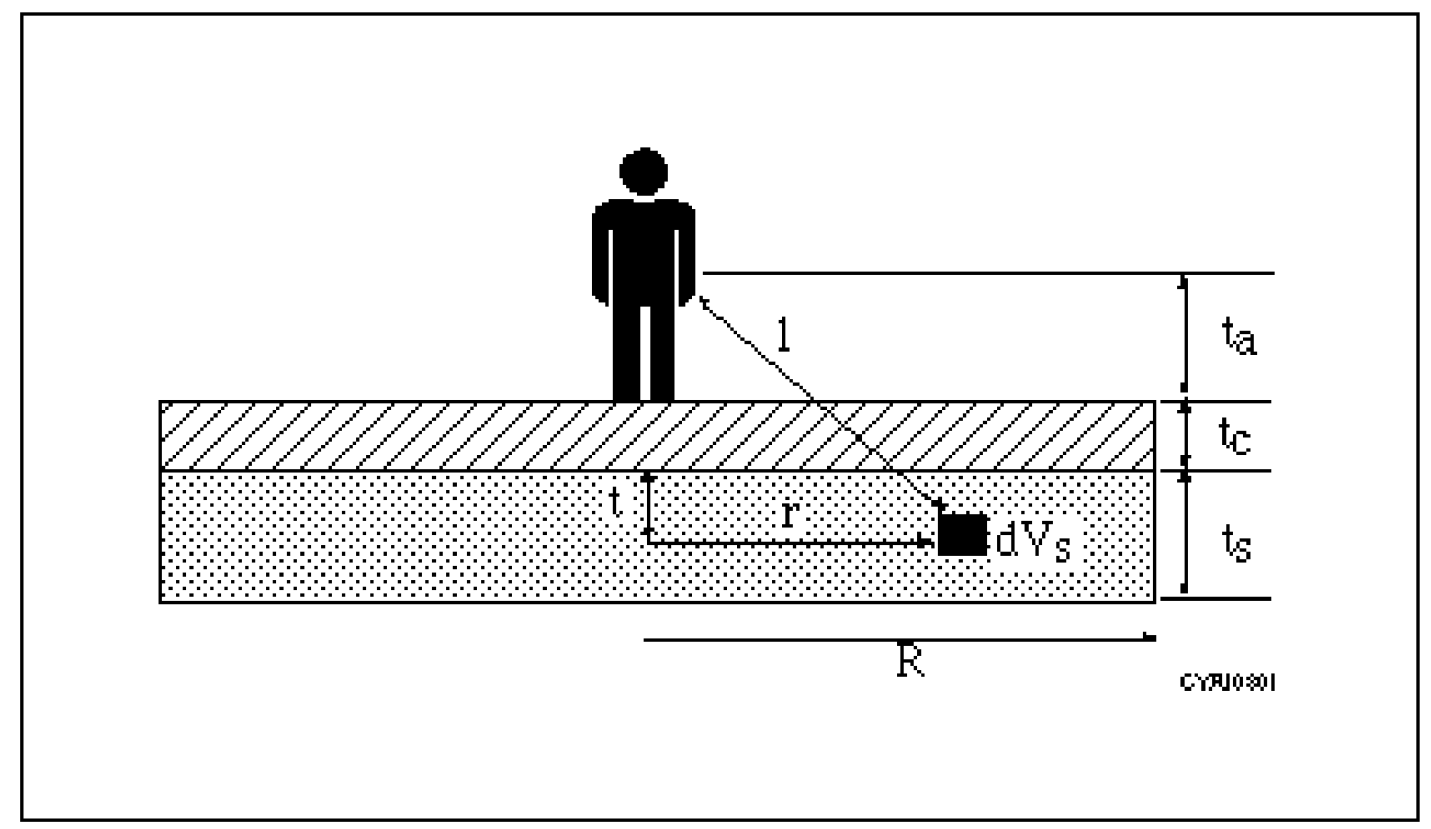

FIGURE 2 Cross Section of Exposure Geometry Showing Element of Integration for Area Factor Calculation 
The volume integral can be written more explicitly as:

$$
\mathrm{m}_{0}^{d t} \mathrm{~m}_{0}^{t_{s}} \frac{e^{\varepsilon} B(z)}{4 \mathrm{~B} l^{2}} 2 \mathrm{~B} r d r
$$

Or, noticing that in the inner integral:

$$
\frac{d z}{z}, \frac{d l}{l}, \frac{r d r}{l^{2}}
$$

then the volume integral can be written:

$$
\frac{1}{2} \mathrm{~m}_{0}^{t_{s}} d t \mathrm{~m}_{z_{0}}^{z_{f}} \frac{e^{\&} B(z)}{z} d z
$$

where

$$
\begin{aligned}
& z_{0}{ }^{\prime} \mu_{a} t_{a} \% \mu_{c} t_{c} \% \mu_{s} t ; \text { and } \\
& z_{f}{ }^{\prime} z_{0} \sqrt{1 \% \frac{R^{2}}{\left(t_{a} \% t_{c} \% t\right)^{2}}}
\end{aligned}
$$

To conserve computational time without sacrificing too much accuracy, the International Commission on Radiological Protection (ICRP) Publication 38 (ICRP 1983) photon energies and yields were condensed into a smaller number of energies and yields for each radionuclide. The spectra-condensing algorithms, which conserve energy, repeatedly combine the photons that are closest in their energies (using their ratio). The yield of the resultant photon is the sum of the yields of the two photons, and the energy is the yield-weighted energy of the two photons. This combining of pairs of photons was repeated until individual photon energy was more than a factor of 3 apart from any other photon energy. This process resulted in four or fewer collapsed photons for all radionuclides processed. It was found that adding extra energy groups beyond four groups would not change area factor more than 5\% for all radionuclides included in the RESRAD database. Even when there are four energy groups, the external dose routine in the RESRAD code is the most timeconsuming routine for most radionuclides. The resultant collapsed gamma energies with their respective fractions for 84 radionuclides are shown in Table 5. 
TABLE 5 Number of Collapsed Gammas (NPT), Energies (EPTs) (in MeV), and Their Respective Gamma Fractions (FPTs) for 84 Radionuclides

\begin{tabular}{|c|c|c|c|c|c|c|c|c|c|}
\hline Radionuclide $^{\mathrm{a}}$ & NPT & EPT(1) & EPT(2) & EPT(3) & ЕРT(4) & FPT(1) & FPT(2) & FPT(3) & FPT(4) \\
\hline $\mathrm{H}-3$ & 0 & 0.00 & 0.00 & 0.00 & 0.00 & 0.00 & 0.00 & 0.00 & 0.00 \\
\hline C-14 & 0 & 0.00 & 0.00 & 0.00 & 0.00 & 0.00 & 0.00 & 0.00 & 0.00 \\
\hline $\mathrm{Na}-22$ & 2 & $8.49 \times 10^{-4}$ & $7.84 \times 10^{-1}$ & 0.00 & 0.00 & $1.25 \times 10^{-3}$ & 2.80 & 0.00 & 0.00 \\
\hline $\mathrm{Al}-26$ & 2 & $5.11 \times 10^{-1}$ & 1.80 & 0.00 & 0.00 & 1.64 & 1.03 & 0.00 & 0.00 \\
\hline S-35 & 0 & 0.00 & 0.00 & 0.00 & 0.00 & 0.00 & 0.00 & 0.00 & 0.00 \\
\hline $\mathrm{Cl}-36$ & 2 & $2.31 \times 10^{-3}$ & $5.11 \times 10^{-1}$ & 0.00 & 0.00 & $1.23 \times 10^{-3}$ & $2.97 \times 10^{-4}$ & 0.00 & 0.00 \\
\hline $\mathrm{K}-40$ & 2 & $2.98 \times 10^{-3}$ & 1.46 & 0.00 & 0.00 & $9.59 \times 10^{-3}$ & $1.07 \times 10^{-1}$ & 0.00 & 0.00 \\
\hline $\mathrm{Ca}-41$ & 1 & $3.31 \times 10^{-3}$ & 0.00 & 0.00 & 0.00 & $1.23 \times 10^{-1}$ & 0.00 & 0.00 & 0.00 \\
\hline $\mathrm{Ca}-45$ & 2 & $4.13 \times 10^{-3}$ & $1.25 \times 10^{-2}$ & 0.00 & 0.00 & $2.49 \times 10^{-6}$ & $2.66 \times 10^{-6}$ & 0.00 & 0.00 \\
\hline Sc-46 & 1 & 1.01 & 0.00 & 0.00 & 0.00 & 2.00 & 0.00 & 0.00 & 0.00 \\
\hline $\mathrm{Mn}-54$ & 1 & $8.35 \times 10^{-1}$ & 0.00 & 0.00 & 0.00 & 1.00 & 0.00 & 0.00 & 0.00 \\
\hline $\mathrm{Fe}-55$ & 1 & $5.97 \times 10^{-3}$ & 0.00 & 0.00 & 0.00 & $2.83 \times 10^{-1}$ & 0.00 & 0.00 & 0.00 \\
\hline $\mathrm{Fe}-59$ & 2 & $1.80 \times 10^{-1}$ & 1.18 & 0.00 & 0.00 & $4.00 \times 10^{-2}$ & $9.97 \times 10^{-1}$ & 0.00 & 0.00 \\
\hline $\mathrm{Co}-57$ & 3 & $7.58 \times 10^{-3}$ & $1.24 \times 10^{-1}$ & $6.92 \times 10^{-1}$ & 0.00 & $6.63 \times 10^{-1}$ & $9.62 \times 10^{-1}$ & $1.60 \times 10^{-3}$ & 0.00 \\
\hline Co-60 & 1 & 1.25 & 0.00 & 0.00 & 0.00 & 2.00 & 0.00 & 0.00 & 0.00 \\
\hline $\mathrm{Ni}-59$ & 1 & $7.01 \times 10^{-3}$ & 0.00 & 0.00 & 0.00 & $3.43 \times 10^{-1}$ & 0.00 & 0.00 & 0.00 \\
\hline $\mathrm{Ni}-63$ & 0 & 0.00 & 0.00 & 0.00 & 0.00 & 0.00 & 0.00 & 0.00 & 0.00 \\
\hline $\mathrm{Zn}-65$ & 2 & $8.04 \times 10^{-3}$ & 1.08 & 0.00 & 0.00 & $3.41 \times 10^{-1}$ & $5.36 \times 10^{-1}$ & 0.00 & 0.00 \\
\hline Ge-68+D & 2 & $9.24 \times 10^{-3}$ & $5.23 \times 10^{-1}$ & 0.00 & 0.00 & $3.86 \times 10^{-1}$ & 1.82 & 0.00 & 0.00 \\
\hline Se-75 & 2 & $1.06 \times 10^{-2}$ & $2.14 \times 10^{-1}$ & 0.00 & 0.00 & $5.28 \times 10^{-1}$ & 1.82 & 0.00 & 0.00 \\
\hline Sr-85 & 2 & $1.35 \times 10^{-2}$ & $5.14 \times 10^{-1}$ & 0.00 & 0.00 & $5.52 \times 10^{-1}$ & $9.80 \times 10^{-1}$ & 0.00 & 0.00 \\
\hline Sr-89 & 1 & $9.09 \times 10^{-1}$ & 0.00 & 0.00 & 0.00 & $9.30 \times 10^{-5}$ & 0.00 & 0.00 & 0.00 \\
\hline Sr-90+D & 2 & $2.08 \times 10^{-3}$ & $1.61 \times 10^{-2}$ & 0.00 & 0.00 & $3.90 \times 10^{-6}$ & $1.05 \times 10^{-4}$ & 0.00 & 0.00 \\
\hline $\mathrm{Zr}-95+\mathrm{D}$ & 3 & $1.69 \times 10^{-2}$ & $2.35 \times 10^{-1}$ & $7.42 \times 10^{-1}$ & 0.00 & $3.11 \times 10^{-3}$ & $1.81 \times 10^{-3}$ & $9.95 \times 10^{-1}$ & 0.00 \\
\hline Nb-94 & 1 & $7.87 \times 10^{-1}$ & 0.00 & 0.00 & 0.00 & 2.00 & 0.00 & 0.00 & 0.00 \\
\hline $\mathrm{Nb}-95$ & 1 & $7.66 \times 10^{-1}$ & 0.00 & 0.00 & 0.00 & 1.00 & 0.00 & 0.00 & 0.00 \\
\hline Tc-99 & 0 & 0.00 & 0.00 & 0.00 & 0.00 & 0.00 & 0.00 & 0.00 & 0.00 \\
\hline Ru-106 & 1 & $5.93 \times 10^{-1}$ & 0.00 & 0.00 & 0.00 & $3.39 \times 10^{-1}$ & 0.00 & 0.00 & 0.00 \\
\hline Ag- $108 \mathrm{~m}$ & 3 & $2.11 \times 10^{-2}$ & $7.92 \times 10^{-2}$ & $5.91 \times 10^{-1}$ & 0.00 & $5.30 \times 10^{-1}$ & $6.78 \times 10^{-2}$ & 2.72 & 0.00 \\
\hline $\mathrm{Ag}-110 \mathrm{~m}+\mathrm{D}$ & 1 & $8.57 \times 10^{-1}$ & 0.00 & 0.00 & 0.00 & 3.19 & 0.00 & 0.00 & 0.00 \\
\hline Cd-109 & 2 & $2.26 \times 10^{-2}$ & $8.80 \times 10^{-2}$ & 0.00 & 0.00 & 1.01 & $3.61 \times 10^{-2}$ & 0.00 & 0.00 \\
\hline Sn-113+D & 2 & $2.47 \times 10^{-2}$ & $3.88 \times 10^{-1}$ & 0.00 & 0.00 & $9.62 \times 10^{-1}$ & $6.61 \times 10^{-1}$ & 0.00 & 0.00 \\
\hline Sb-124 & 1 & $9.83 \times 10^{-1}$ & 0.00 & 0.00 & 0.00 & 1.83 & 0.00 & 0.00 & 0.00 \\
\hline Sb-125 & 2 & $2.87 \times 10^{-2}$ & $4.88 \times 10^{-1}$ & 0.00 & 0.00 & $5.13 \times 10^{-1}$ & $8.49 \times 10^{-1}$ & 0.00 & 0.00 \\
\hline $\mathrm{Te}-125 \mathrm{~m}$ & 2 & $2.85 \times 10^{-2}$ & $1.09 \times 10^{-1}$ & 0.00 & 0.00 & 1.22 & $2.74 \times 10^{-3}$ & 0.00 & 0.00 \\
\hline $\mathrm{I}-125$ & 1 & $2.84 \times 10^{-2}$ & 0.00 & 0.00 & 0.00 & 1.46 & 0.00 & 0.00 & 0.00 \\
\hline I-129 & 2 & $4.29 \times 10^{-3}$ & $3.13 \times 10^{-2}$ & 0.00 & 0.00 & $6.60 \times 10^{-2}$ & $7.75 \times 10^{-1}$ & 0.00 & 0.00 \\
\hline Cs-134 & 1 & $6.98 \times 10^{-1}$ & 0.00 & 0.00 & 0.00 & 2.23 & 0.00 & 0.00 & 0.00 \\
\hline Cs-135 & 0 & 0.00 & 0.00 & 0.00 & 0.00 & 0.00 & 0.00 & 0.00 & 0.00 \\
\hline Cs-137+D & 2 & $3.21 \times 10^{-2}$ & $6.62 \times 10^{-1}$ & 0.00 & 0.00 & $5.72 \times 10^{-2}$ & $8.50 \times 10^{-1}$ & 0.00 & 0.00 \\
\hline $\mathrm{Ce}-141$ & 2 & $3.69 \times 10^{-2}$ & $1.45 \times 10^{-1}$ & 0.00 & 0.00 & $1.70 \times 10^{-1}$ & $4.80 \times 10^{-1}$ & 0.00 & 0.00 \\
\hline Ce-144+D & 3 & $3.70 \times 10^{-2}$ & $1.24 \times 10^{-1}$ & 1.24 & 0.00 & $1.17 \times 10^{-1}$ & $1.33 \times 10^{-1}$ & $2.56 \times 10^{-2}$ & 0.00 \\
\hline Pm-147 & 1 & $8.64 \times 10^{-2}$ & 0.00 & 0.00 & 0.00 & $5.03 \times 10^{-5}$ & 0.00 & 0.00 & 0.00 \\
\hline Sm-147 & 0 & 0.00 & 0.00 & 0.00 & 0.00 & 0.00 & 0.00 & 0.00 & 0.00 \\
\hline Sm-151 & 2 & $6.49 \times 10^{-3}$ & $2.15 \times 10^{-2}$ & 0.00 & 0.00 & $1.10 \times 10^{-3}$ & $2.93 \times 10^{-4}$ & 0.00 & 0.00 \\
\hline Eu-152 & 2 & $6.34 \times 10^{-2}$ & $8.47 \times 10^{-1}$ & 0.00 & 0.00 & 1.03 & 1.27 & 0.00 & 0.00 \\
\hline Eu-154 & 3 & $4.33 \times 10^{-2}$ & $1.41 \times 10^{-1}$ & 1.01 & 0.00 & $2.48 \times 10^{-1}$ & $4.71 \times 10^{-1}$ & 1.14 & 0.00 \\
\hline Eu-155 & 2 & $6.42 \times 10^{-3}$ & $7.76 \times 10^{-2}$ & 0.00 & 0.00 & $6.34 \times 10^{-2}$ & $7.75 \times 10^{-1}$ & 0.00 & 0.00 \\
\hline Gd-152 & 0 & 0.00 & 0.00 & 0.00 & 0.00 & 0.00 & 0.00 & 0.00 & 0.00 \\
\hline Gd-153 & 1 & $5.95 \times 10^{-2}$ & 0.00 & 0.00 & 0.00 & 1.75 & 0.00 & 0.00 & 0.00 \\
\hline Тa-182 & 2 & $1.01 \times 10^{-1}$ & 1.18 & 0.00 & 0.00 & 1.27 & $9.85 \times 10^{-1}$ & 0.00 & 0.00 \\
\hline Ir-192 & 2 & $6.55 \times 10^{-2}$ & $3.72 \times 10^{-1}$ & 0.00 & 0.00 & $9.43 \times 10^{-2}$ & 2.16 & 0.00 & 0.00 \\
\hline $\mathrm{Au}-195$ & 1 & $7.16 \times 10^{-2}$ & 0.00 & 0.00 & 0.00 & 1.10 & 0.00 & 0.00 & 0.00 \\
\hline Tl-204 & 1 & $7.24 \times 10^{-2}$ & 0.00 & 0.00 & 0.00 & $1.45 \times 10^{-2}$ & 0.00 & 0.00 & 0.00 \\
\hline $\mathrm{Pb}-210+\mathrm{D}$ & 2 & $1.24 \times 10^{-2}$ & $4.65 \times 10^{-2}$ & 0.00 & 0.00 & $2.37 \times 10^{-1}$ & $4.05 \times 10^{-2}$ & 0.00 & 0.00 \\
\hline Bi-207 & 2 & $7.66 \times 10^{-2}$ & $8.22 \times 10^{-1}$ & 0.00 & 0.00 & $7.44 \times 10^{-1}$ & 1.80 & 0.00 & 0.00 \\
\hline Po-210 & 1 & $8.02 \times 10^{-1}$ & 0.00 & 0.00 & 0.00 & $1.06 \times 10^{-5}$ & 0.00 & 0.00 & 0.00 \\
\hline $\mathrm{Ra}-226+\mathrm{D}$ & 4 & $2.67 \times 10^{-2}$ & $9.30 \times 10^{-2}$ & $4.81 \times 10^{-1}$ & 1.53 & $1.38 \times 10^{-1}$ & $2.46 \times 10^{-1}$ & 1.27 & $6.79 \times 10^{-1}$ \\
\hline $\mathrm{Ra}-228+\mathrm{D}$ & 3 & $1.49 \times 10^{-2}$ & $3.01 \times 10^{-1}$ & 1.01 & 0.00 & $3.58 \times 10^{-1}$ & $4.92 \times 10^{-1}$ & $7.66 \times 10^{-1}$ & 0.00 \\
\hline Ac- $227+\mathrm{D}$ & 3 & $1.40 \times 10^{-2}$ & $9.42 \times 10^{-2}$ & $3.30 \times 10^{-1}$ & 0.00 & $6.41 \times 10^{-1}$ & $9.06 \times 10^{-1}$ & $8.60 \times 10^{-1}$ & 0.00 \\
\hline Th-228+D & 4 & $1.36 \times 10^{-2}$ & $1.77 \times 10^{-1}$ & $6.54 \times 10^{-1}$ & 2.55 & $3.14 \times 10^{-1}$ & $9.47 \times 10^{-1}$ & $6.03 \times 10^{-1}$ & $3.87 \times 10^{-1}$ \\
\hline Th-229+D & 4 & $1.97 \times 10^{-2}$ & $1.16 \times 10^{-1}$ & $4.51 \times 10^{-1}$ & 1.57 & 1.37 & 1.16 & $3.10 \times 10^{-1}$ & $2.12 \times 10^{-2}$ \\
\hline Th-230 & 2 & $1.45 \times 10^{-2}$ & $8.27 \times 10^{-2}$ & 0.00 & 0.00 & $8.10 \times 10^{-2}$ & $4.50 \times 10^{-3}$ & 0.00 & 0.00 \\
\hline Th-232 & 2 & $1.45 \times 10^{-2}$ & $7.21 \times 10^{-2}$ & 0.00 & 0.00 & $8.00 \times 10^{-2}$ & $2.46 \times 10^{-3}$ & 0.00 & 0.00 \\
\hline $\mathrm{Pa}-231$ & 3 & $1.62 \times 10^{-2}$ & $9.09 \times 10^{-2}$ & $2.83 \times 10^{-1}$ & 0.00 & $7.87 \times 10^{-1}$ & $6.95 \times 10^{-3}$ & $1.30 \times 10^{-1}$ & 0.00 \\
\hline
\end{tabular}


TABLE 5 (Cont.)

\begin{tabular}{|c|c|c|c|c|c|c|c|c|c|}
\hline Radionuclide $^{\mathrm{a}}$ & NPT & EPT(1) & $\mathrm{EPT}(2)$ & ЕРТ(3) & ЕРT(4) & FPT(1) & $\mathrm{FPT}(2)$ & FPT(3) & FPT(4) \\
\hline U-232 & 3 & $1.53 \times 10^{-2}$ & $7.75 \times 10^{-2}$ & $2.98 \times 10^{-1}$ & 0.00 & $1.26 \times 10^{-1}$ & $3.03 \times 10^{-3}$ & $7.20 \times 10^{-5}$ & 0.00 \\
\hline U-233 & 2 & $1.59 \times 10^{-2}$ & $1.58 \times 10^{-1}$ & 0.00 & 0.00 & $6.66 \times 10^{-2}$ & $1.60 \times 10^{-3}$ & 0.00 & 0.00 \\
\hline U-234 & 2 & $1.53 \times 10^{-2}$ & $7.13 \times 10^{-2}$ & 0.00 & 0.00 & $1.05 \times 10^{-1}$ & $1.66 \times 10^{-3}$ & 0.00 & 0.00 \\
\hline U-235 & 2 & $1.68 \times 10^{-2}$ & $1.59 \times 10^{-1}$ & 0.00 & 0.00 & 1.14 & 1.01 & 0.00 & 0.00 \\
\hline U-236 & 2 & $1.53 \times 10^{-2}$ & $6.25 \times 10^{-2}$ & 0.00 & 0.00 & $9.87 \times 10^{-2}$ & $9.85 \times 10^{-4}$ & 0.00 & 0.00 \\
\hline $\mathrm{U}-238+\mathrm{D}$ & 3 & $1.55 \times 10^{-2}$ & $8.27 \times 10^{-2}$ & $9.15 \times 10^{-1}$ & 0.00 & $1.91 \times 10^{-1}$ & $1.02 \times 10^{-1}$ & $1.46 \times 10^{-2}$ & 0.00 \\
\hline $\mathrm{Np}-237+\mathrm{D}$ & 3 & $1.76 \times 10^{-2}$ & $9.70 \times 10^{-2}$ & $3.17 \times 10^{-1}$ & 0.00 & 1.15 & $5.87 \times 10^{-1}$ & $5.06 \times 10^{-1}$ & 0.00 \\
\hline Pu-238 & 2 & $1.61 \times 10^{-2}$ & $5.26 \times 10^{-2}$ & 0.00 & 0.00 & $1.11 \times 10^{-1}$ & $4.64 \times 10^{-4}$ & 0.00 & 0.00 \\
\hline $\mathrm{Pu}-239$ & 4 & $7.30 \times 10^{-5}$ & $1.61 \times 10^{-2}$ & $4.88 \times 10^{-2}$ & $1.87 \times 10^{-1}$ & $9.99 \times 10^{-1}$ & $4.17 \times 10^{-2}$ & $2.67 \times 10^{-4}$ & $2.09 \times 10^{-4}$ \\
\hline $\mathrm{Pu}-240$ & 2 & $1.61 \times 10^{-2}$ & $5.32 \times 10^{-2}$ & 0.00 & 0.00 & $1.06 \times 10^{-1}$ & $5.20 \times 10^{-4}$ & 0.00 & 0.00 \\
\hline $\mathrm{Pu}-241$ & 2 & $1.62 \times 10^{-2}$ & $1.12 \times 10^{-1}$ & 0.00 & 0.00 & $7.90 \times 10^{-5}$ & $4.23 \times 10^{-5}$ & 0.00 & 0.00 \\
\hline $\mathrm{Pu}-242$ & 2 & $1.61 \times 10^{-2}$ & $5.54 \times 10^{-2}$ & 0.00 & 0.00 & $8.81 \times 10^{-2}$ & $4.39 \times 10^{-4}$ & 0.00 & 0.00 \\
\hline $\mathrm{Pu}-244$ & 2 & $5.46 \times 10^{-2}$ & $6.48 \times 10^{-1}$ & 0.00 & 0.00 & $2.09 \times 10^{-2}$ & $4.99 \times 10^{-1}$ & 0.00 & 0.00 \\
\hline Am-241 & 2 & $1.68 \times 10^{-2}$ & $5.95 \times 10^{-2}$ & 0.00 & 0.00 & $6.65 \times 10^{-1}$ & $3.57 \times 10^{-1}$ & 0.00 & 0.00 \\
\hline Am-243+D & 2 & $1.92 \times 10^{-2}$ & $1.24 \times 10^{-1}$ & 0.00 & 0.00 & $8.18 \times 10^{-1}$ & 1.71 & 0.00 & 0.00 \\
\hline $\mathrm{Cm}-243$ & 2 & $1.67 \times 10^{-2}$ & $1.60 \times 10^{-1}$ & 0.00 & 0.00 & $5.72 \times 10^{-1}$ & $7.75 \times 10^{-1}$ & 0.00 & 0.00 \\
\hline $\mathrm{Cm}-244$ & 1 & $1.69 \times 10^{-2}$ & 0.00 & 0.00 & 0.00 & $1.00 \times 10^{-1}$ & 0.00 & 0.00 & 0.00 \\
\hline $\mathrm{Cm}-248$ & 1 & $4.40 \times 10^{-2}$ & 0.00 & 0.00 & 0.00 & $2.02 \times 10^{-4}$ & 0.00 & 0.00 & 0.00 \\
\hline Cf-252 & 1 & $7.55 \times 10^{-2}$ & 0.00 & 0.00 & 0.00 & $2.93 \times 10^{-4}$ & 0.00 & 0.00 & 0.00 \\
\hline
\end{tabular}

a + D means that gamma energies of the associated progeny radionuclides with half-lives less than 30 days are included.

The radionuclide-specific area factor for a circular area $\mathrm{x}, F_{A}{ }^{n u c}(\mathrm{x})$, is obtained by combining the energy-dependent area factors weighted by their photon fraction, $F P T_{i}$, and dose contribution at the reference point:

$$
F_{A}^{n u c}(x), \frac{\mathrm{j}_{i} F_{A}\left(E P T_{i}\right) F P T_{i} D_{\text {slab }}\left(E P T_{i}\right)}{\mathrm{j}_{i} F P T_{i} D_{\text {slab }}\left(E P T_{i}\right)},
$$

where

$$
D_{\text {slab }}\left(E P T_{i}\right)=\text { effective dose equivalent from the infinite slab geometry. }
$$

\subsection{SHAPE FACTOR}

A shape factor, $F_{S}$, is used to correct a noncircular-shaped contaminated area on the basis of an ideally circular zone. The shape factor of a circular contaminated area is 1.0. For an irregularly shaped contaminated area, the shape factor is obtained by enclosing the irregularly shaped contaminated area in a circle, multiplying the area factor of each annulus by the fraction of the 
annulus area that is contaminated, summing the products, and dividing by the area factor of a circular contaminated zone that is equivalent in area:

$$
F_{s}, \frac{j_{i^{\prime} 0}^{n} f_{i}\left[F_{A}^{n u c}\left(A_{i}\right) \& F_{A}^{n u c}\left(A_{i \& 1}\right)\right]}{F_{A}^{n u c}\left(j_{i^{\prime} 0}^{n} f_{i}\left(A_{i} \& A_{i \& 1}\right)\right)},
$$

where

$$
f_{i}=\text { fraction of annular area that is contaminated and }
$$

$$
F_{A}^{n u c}(x)=\text { radionuclide specific area factor for an area } \mathrm{x} .
$$




\section{COMPARISON OF RESRAD MODELS (VERSION 5.44 AND EARLIER VS. 5.60 AND LATER)}

The contribution to the effective dose equivalent $(E D E)$ rate from the external ground radiation of the source of any depth, shape, cover, and size for a single radionuclide can be given by the equation:

$$
E D E=\text { Source Activity } \times D C F \times F_{C D} \times F_{A}^{n u c} \times F_{S},
$$

where $D C F$ is the dose conversion factor for the radionuclide present at the unit concentration in a uniformly contaminated zone of infinite depth and lateral extent. The $D C F$ multiplied by the source activity gives the $E D E$ for the given activity. When this value is multiplied by $F_{C D}$, the dose equivalent is obtained for a geometry with a given source depth and cover thickness but still of an infinite lateral extent. $F_{A}{ }^{n u c}$ accounts for the finite radius source, but the source is still assumed to be circular. $F_{S}$ accounts for irregular source shapes. If many radionuclides are present, the $E D E$ can be calculated separately for each radionuclide and summed to get the total $E D E$.

\subsection{DOSE CONVERSION FACTORS}

Table 6 compares the infinite thickness $D C F$ s used in the FGR-12 report with those used in the previous RESRAD model for the 67 (six-month equilibration) and 84 (30-day equilibration) radionuclides. The $D C F$ s used in RESRAD 5.44 and earlier versions were based on U.S. Department

of Energy (DOE) report EH-0070 (DOE 1988) and the methods of Kocher and Sjoreen (1985) and Chen (1991). The "Ratio" columns represent the ratio of previous RESRAD DCFs divided by the FGR-12 DCFs. In the table, "BZ" indicates cases in which both values are zero, "INF" indicates radionuclides for which the FGR values are zero and RESRAD has finite values, and "ZERO" indicates radionuclides for which only RESRAD values are zero.

The following comparisons are made:

- For Ca-41, Fe-55, and Ni-59, FGR-12 DCFs are zero, and the previous RESRAD model had nonzero values.

- For C-14, S-35, Sr-90, and Cs-135, FGR-12 assigns some finite $D C F$, whereas the previous RESRAD model had zero values.

- For H-3, Ni-63, Sm-147, and Gd-152, DCFs are zero in both cases.

- For 38 out of 84 radionuclides, differences are equal to or less than $20 \%$. 
TABLE 6 Comparison of Infinite Thickness DCFs $[(\mathrm{mrem} / \mathrm{year}) /(\mathrm{pCi} / \mathrm{g})]$ between the Previous RESRAD Model and FGR-12

\begin{tabular}{|c|c|c|c|c|c|c|c|}
\hline \multirow[b]{2}{*}{ Radionuclide } & \multicolumn{3}{|c|}{ 6-Month Equilibration } & \multirow[b]{2}{*}{ Radionuclide } & \multicolumn{3}{|c|}{ 30-Day Equilibration } \\
\hline & RESRAD & FGR-12 & Ratio $^{\mathrm{a}}$ & & RESRAD & FGR-12 & Ratio $^{a}$ \\
\hline $\mathrm{H}-3$ & 0.00 & 0.00 & $\mathrm{BZ}^{\mathrm{b}}$ & $\mathrm{H}-3$ & 0.00 & 0.00 & $\mathrm{BZ}$ \\
\hline C-14 & 0.00 & $1.34 \times 10^{-5}$ & $\mathrm{ZERO}^{\mathrm{c}}$ & C-14 & 0.00 & $1.34 \times 10^{-5}$ & ZERO \\
\hline $\mathrm{Na}-22$ & $1.54 \times 10^{1}$ & $1.37 \times 10^{1}$ & 1.12 & $\mathrm{Na}-22$ & $1.54 \times 10^{1}$ & $1.37 \times 10^{1}$ & 1.12 \\
\hline $\mathrm{Al}-26$ & $2.00 \times 10^{1}$ & $1.74 \times 10^{1}$ & 1.15 & Al-26 & $2.00 \times 10^{1}$ & $1.74 \times 10^{1}$ & 1.15 \\
\hline $\mathrm{Cl}-36$ & $1.02 \times 10^{-3}$ & $2.39 \times 10^{-3}$ & $4.31 \times 10^{-1}$ & $\mathrm{~S}-35$ & 0.00 & $1.49 \times 10^{-5}$ & ZERO \\
\hline K-40 & 1.17 & 1.04 & 1.13 & $\mathrm{Cl}-36$ & $1.02 \times 10^{-3}$ & $2.39 \times 10^{-3}$ & $4.31 \times 10^{-1}$ \\
\hline $\mathrm{Ca}-41$ & $1.94 \times 10^{-6}$ & 0.00 & $\mathrm{INF}^{\mathrm{d}}$ & $\mathrm{K}-40$ & 1.17 & 1.04 & 1.13 \\
\hline Mn-54 & 5.88 & 5.16 & 1.14 & $\mathrm{Ca}-41$ & $1.94 \times 10^{-6}$ & 0.00 & $\mathrm{INF}^{\mathrm{d}}$ \\
\hline $\mathrm{Fe}-55$ & $4.44 \times 10^{-6}$ & 0.00 & INF & $\mathrm{Ca}-45$ & $2.53 \mathrm{E}-10$ & $6.26 \times 10^{-5}$ & $4.04 \times 10^{-6}$ \\
\hline Co-57 & $5.03 \times 10^{-1}$ & $5.01 \times 10^{-1}$ & 1.00 & Sc-46 & $1.33 \times 10^{1}$ & $1.27 \times 10^{1}$ & 1.05 \\
\hline Co-60 & $2.27 \times 10^{1}$ & $1.62 \times 10^{1}$ & 1.40 & Mn-54 & 5.88 & 5.16 & 1.14 \\
\hline $\mathrm{Ni}-59$ & $5.38 \times 10^{-6}$ & 0.00 & INF & $\mathrm{Fe}-55$ & $4.44 \times 10^{-6}$ & 0.00 & INF \\
\hline $\mathrm{Ni}-63$ & 0.00 & 0.00 & BZ & $\mathrm{Fe}-59$ & 7.99 & 7.64 & 1.05 \\
\hline $\mathrm{Zn}-65$ & 3.71 & 3.70 & 1.00 & Co-57 & $5.03 \times 10^{-1}$ & $5.01 \times 10^{-1}$ & 1.00 \\
\hline Ge-68+D180 & 6.39 & 5.62 & 1.14 & Co-60 & $2.27 \times 10^{1}$ & $1.62 \times 10^{1}$ & 1.40 \\
\hline Sr-90+D180 & 0.00 & $2.46 \times 10^{-2}$ & ZERO & $\mathrm{Ni}-59$ & $5.38 \times 10^{-6}$ & 0.00 & INF \\
\hline Nb-94 & $1.42 \times 10^{1}$ & 9.68 & 1.46 & $\mathrm{Ni}-63$ & 0.00 & 0.00 & $\mathrm{BZ}$ \\
\hline Tc-99 & $1.68 \times 10^{-6}$ & $1.26 \times 10^{-4}$ & $1.33 \times 10^{-2}$ & Zn-65 & 3.71 & 3.70 & 1.00 \\
\hline Ru-106 & 1.35 & 1.29 & 1.05 & Ge- $68+D 30^{\mathrm{f}}$ & 6.39 & 5.62 & 1.14 \\
\hline Ag- $108 \mathrm{~m}$ & $1.11 \times 10^{1}$ & 9.65 & 1.15 & Se-75 & 2.17 & 1.98 & 1.10 \\
\hline $\mathrm{Ag}-110 \mathrm{~m}$ & $1.92 \times 10^{1}$ & $1.72 \times 10^{1}$ & 1.12 & $\mathrm{Sr}-85$ & 3.40 & 2.97 & 1.14 \\
\hline Cd-109 & $1.17 \times 10^{-2}$ & $1.47 \times 10^{-2}$ & $7.96 \times 10^{-1}$ & Sr-89 & $5.77 \times 10^{-4}$ & $9.08 \times 10^{-3}$ & $6.35 \times 10^{-2}$ \\
\hline Sb-125+D180 & 2.81 & 2.45 & 1.15 & Sr-90+D30 & 0.00 & $2.46 \times 10^{-2}$ & ZERO \\
\hline I-129 & $3.24 \times 10^{-2}$ & $1.29 \times 10^{-2}$ & 2.51 & Zr-95+D30 & 5.15 & 4.52 & 1.14 \\
\hline Cs-134 & $1.08 \times 10^{1}$ & 9.47 & 1.14 & $\mathrm{Nb}-94$ & $1.42 \times 10^{1}$ & 9.68 & 1.47 \\
\hline Cs-135 & 0.00 & $3.83 \times 10^{-5}$ & ZERO & $\mathrm{Nb}-95$ & 5.36 & 4.69 & 1.14 \\
\hline Cs-137+D180 & 5.03 & 3.41 & 1.48 & Tc-99 & $1.68 \times 10^{-6}$ & $1.26 \times 10^{-4}$ & $1.33 \times 10^{-2}$ \\
\hline Ce-144+D180 & $3.12 \times 10^{-1}$ & $3.20 \times 10^{-1}$ & $9.75 \times 10^{-1}$ & Ru-106+D30 & 1.35 & 1.29 & 1.05 \\
\hline Pm-147 & $1.47 \times 10^{-5}$ & $5.01 \times 10^{-5}$ & $2.93 \times 10^{-1}$ & Ag-108m+D30 & $1.11 \times 10^{1}$ & 9.65 & 1.15 \\
\hline Sm-147 & 0.00 & 0.00 & BZ & $\mathrm{Ag}-110 \mathrm{~m}+\mathrm{D} 30$ & $1.92 \times 10^{1}$ & $1.72 \times 10^{1}$ & 1.12 \\
\hline Sm-151 & $5.45 \times 10^{-7}$ & $9.84 \times 10^{-7}$ & $5.54 \times 10^{-1}$ & Cd-109 & $1.17 \times 10^{-2}$ & $1.47 \times 10^{-2}$ & $7.96 \times 10^{-1}$ \\
\hline Eu-152 & 9.91 & 7.01 & 1.41 & Sn-113+D30 & 1.73 & 1.46 & 1.18 \\
\hline Eu-154 & $1.10 \times 10^{1}$ & 7.68 & 1.43 & Sb-124 & $1.32 \times 10^{1}$ & $1.17 \times 10^{1}$ & 1.13 \\
\hline Eu-155 & $1.65 \times 10^{-1}$ & $1.82 \times 10^{-1}$ & $9.07 \times 10^{-1}$ & Sb-125 & 2.81 & 2.45 & 1.15 \\
\hline Gd-152 & 0.00 & 0.00 & BZ & $\mathrm{Te}-125 \mathrm{~m}$ & $8.80 \times 10^{-3}$ & $1.51 \times 10^{-2}$ & $5.83 \times 10^{-1}$ \\
\hline Gd-153 & $2.12 \times 10^{-1}$ & $2.45 \times 10^{-1}$ & $8.65 \times 10^{-1}$ & $\mathrm{I}-125$ & $9.07 \times 10^{-3}$ & $1.66 \times 10^{-2}$ & $5.46 \times 10^{-1}$ \\
\hline Au-195 & $1.67 \times 10^{-1}$ & $2.07 \times 10^{-1}$ & $8.07 \times 10^{-1}$ & I- 129 & $3.24 \times 10^{-2}$ & $1.29 \times 10^{-2}$ & 2.51 \\
\hline $\mathrm{Tl}-204$ & $2.20 \times 10^{-3}$ & $4.05 \times 10^{-3}$ & $5.43 \times 10^{-1}$ & Cs-134 & $1.08 \times 10^{1}$ & 9.47 & 1.14 \\
\hline $\mathrm{Pb}-210+\mathrm{D} 180$ & $4.87 \times 10^{-3}$ & $6.12 \times 10^{-3}$ & $7.96 \times 10^{-1}$ & Cs-135 & 0.00 & $3.83 \times 10^{-5}$ & ZERO \\
\hline Bi-207 & 9.72 & 9.38 & 1.04 & Cs-137+D30 & 5.03 & 3.41 & 1.48 \\
\hline Ra-226+D180 & $1.55 \times 10^{1}$ & $1.12 \times 10^{1}$ & 1.38 & $\mathrm{Ce}-141$ & $3.20 \times 10^{-1}$ & $3.18 \times 10^{-1}$ & 1.01 \\
\hline Ra-228+D180 & 8.18 & 5.98 & 1.37 & Ce-144+D30 & $3.12 \times 10^{-1}$ & $3.20 \times 10^{-1}$ & $9.75 \times 10^{-1}$ \\
\hline Ac- $227+D 180$ & 2.76 & 2.01 & 1.37 & Pm-147 & $1.47 \times 10^{-5}$ & $5.01 \times 10^{-5}$ & $2.93 \times 10^{-1}$ \\
\hline Th-228+D180 & $1.33 \times 10^{1}$ & $1.02 \times 10^{1}$ & 1.30 & Sm-147 & 0.00 & 0.00 & BZ \\
\hline Th-229+D180 & 2.20 & 1.60 & 1.38 & Sm-151 & $5.45 \times 10^{-7}$ & $9.84 \times 10^{-7}$ & $5.54 \times 10^{-1}$ \\
\hline Th-230 & $2.11 \times 10^{-3}$ & $1.21 \times 10^{-3}$ & 1.74 & Eu-152 & 9.91 & 7.01 & 1.41 \\
\hline Th-232 & $1.35 \times 10^{-3}$ & $5.21 \times 10^{-4}$ & 2.59 & Eu-154 & $1.10 \times 10^{1}$ & 7.68 & 1.43 \\
\hline $\mathrm{Pa}-231$ & $2.21 \times 10^{-1}$ & $1.91 \times 10^{-1}$ & 1.16 & Eu-155 & $1.65 \times 10^{-1}$ & $1.82 \times 10^{-1}$ & $9.07 \times 10^{-1}$ \\
\hline
\end{tabular}


TABLE 6 (Cont.)

\begin{tabular}{|c|c|c|c|c|c|c|c|}
\hline \multirow[b]{2}{*}{ Radionuclide } & \multicolumn{3}{|c|}{ 6-Month Equilibration } & \multirow[b]{2}{*}{ Radionuclide } & \multicolumn{3}{|c|}{ 30-Day Equilibration } \\
\hline & RESRAD & FGR-12 & Ratio $^{\mathrm{a}}$ & & RESRAD & FGR-12 & Ratio $^{a}$ \\
\hline $\mathrm{U}-232$ & $2.19 \times 10^{-3}$ & $9.02 \times 10^{-4}$ & 2.43 & Gd-152 & 0.00 & 0.00 & BZ \\
\hline $\mathrm{U}-233$ & $1.40 \times 10^{-3}$ & $1.40 \times 10^{-3}$ & 1.00 & Gd-153 & $2.12 \times 10^{-1}$ & $2.45 \times 10^{-1}$ & $8.65 \times 10^{-1}$ \\
\hline $\mathrm{U}-234$ & $1.58 \times 10^{-3}$ & $4.02 \times 10^{-4}$ & 3.92 & Ta-182 & 8.26 & 7.94 & 1.04 \\
\hline $\mathrm{U}-235+\mathrm{D} 180$ & $8.94 \times 10^{-1}$ & $7.57 \times 10^{-1}$ & 1.18 & Ir-192 & 5.22 & 4.61 & 1.13 \\
\hline $\mathrm{U}-236$ & $1.35 \times 10^{-3}$ & $2.15 \times 10^{-4}$ & 6.28 & Au-195 & $1.67 \times 10^{-1}$ & $2.07 \times 10^{-1}$ & $8.07 \times 10^{-1}$ \\
\hline $\mathrm{U}-238+\mathrm{D} 180$ & $1.27 \times 10^{-1}$ & $1.37 \times 10^{-1}$ & $9.27 \times 10^{-1}$ & Tl-204 & $2.20 \times 10^{-3}$ & $4.05 \times 10^{-3}$ & $5.43 \times 10^{-1}$ \\
\hline Np-237+D180 & 1.61 & 1.10 & 1.46 & $\mathrm{~Pb}-210+\mathrm{D} 30$ & $4.81 \times 10^{-3}$ & $6.05 \times 10^{-3}$ & $7.95 \times 10^{-1}$ \\
\hline $\mathrm{Pu}-238$ & $1.56 \times 10^{-3}$ & $1.51 \times 10^{-4}$ & $1.03 \times 10^{1}$ & $\mathrm{Bi}-207$ & 9.72 & 9.38 & 1.04 \\
\hline $\mathrm{Pu}-239$ & $8.14 \times 10^{-4}$ & $2.95 \times 10^{-4}$ & 2.75 & Po-210 & $5.98 \times 10^{-5}$ & $5.23 \times 10^{-5}$ & 1.14 \\
\hline $\mathrm{Pu}-240$ & $1.48 \times 10^{-3}$ & $1.47 \times 10^{-4}$ & $1.01 \times 10^{1}$ & $\mathrm{Ra}-226+\mathrm{D} 30$ & $1.55 \times 10^{1}$ & $1.12 \times 10^{1}$ & 1.38 \\
\hline $\mathrm{Pu}-241+\mathrm{D} 180$ & $1.88 \times 10^{-5}$ & $1.89 \times 10^{-5}$ & $9.95 \times 10^{-1}$ & $\mathrm{Ra}-228+\mathrm{D} 30$ & 8.18 & 5.98 & 1.37 \\
\hline $\mathrm{Pu}-242$ & $1.24 \times 10^{-3}$ & $1.28 \times 10^{-4}$ & 9.69 & Ac-227+D30 & 2.76 & 2.01 & 1.37 \\
\hline $\mathrm{Pu}-244+\mathrm{D} 180$ & 2.23 & 7.73 & $2.88 \times 10^{-1}$ & Th-228+D30 & $1.33 \times 10^{1}$ & $1.02 \times 10^{1}$ & 1.30 \\
\hline Am-241 & $4.79 \times 10^{-2}$ & $4.37 \times 10^{-2}$ & 1.10 & Th-229+D30 & 2.20 & 1.60 & 1.38 \\
\hline Am-243+D180 & 1.08 & $8.95 \times 10^{-1}$ & 1.21 & Th-230 & $2.11 \times 10^{-3}$ & $1.21 \times 10^{-3}$ & 1.74 \\
\hline $\mathrm{Cm}-243$ & $7.26 \times 10^{-1}$ & $5.83 \times 10^{-1}$ & 1.25 & Th-232 & $1.35 \times 10^{-3}$ & $5.21 \times 10^{-4}$ & 2.59 \\
\hline $\mathrm{Cm}-244$ & $1.51 \times 10^{-3}$ & $1.26 \times 10^{-4}$ & $1.20 \times 10^{1}$ & $\mathrm{~Pa}-231$ & $2.21 \times 10^{-1}$ & $1.91 \times 10^{-1}$ & 1.16 \\
\hline $\mathrm{Cm}-248$ & $6.10 \times 10^{-6}$ & $8.78 \times 10^{-5}$ & $6.95 \times 10^{-2}$ & $\mathrm{U}-232$ & $2.19 \times 10^{-3}$ & $9.02 \times 10^{-4}$ & 2.43 \\
\hline \multirow[t]{18}{*}{ Cf- 252} & $6.32 \times 10^{-5}$ & $1.76 \times 10^{-4}$ & $3.59 \times 10^{-1}$ & U-233 & $1.40 \times 10^{-3}$ & $1.40 \times 10^{-3}$ & 1.00 \\
\hline & & & & U-234 & $1.58 \times 10^{-3}$ & $4.02 \times 10^{-4}$ & 3.93 \\
\hline & & & & $\mathrm{U}-235+\mathrm{D} 30$ & $8.94 \times 10^{-1}$ & $7.57 \times 10^{-1}$ & 1.18 \\
\hline & & & & U-236 & $1.35 \times 10^{-3}$ & $2.15 \times 10^{-4}$ & 6.28 \\
\hline & & & & $\mathrm{U}-238+\mathrm{D} 30$ & $1.27 \times 10^{-1}$ & $1.37 \times 10^{-1}$ & $9.27 \times 10^{-1}$ \\
\hline & & & & $\mathrm{Np}-237+\mathrm{D} 30$ & 1.61 & 1.10 & 1.46 \\
\hline & & & & $\mathrm{Pu}-238$ & $1.56 \times 10^{-3}$ & $1.51 \times 10^{-4}$ & $1.03 \times 10^{1}$ \\
\hline & & & & $\mathrm{Pu}-239$ & $8.14 \times 10^{-4}$ & $2.95 \times 10^{-4}$ & 2.76 \\
\hline & & & & $\mathrm{Pu}-240$ & $1.48 \times 10^{-3}$ & $1.47 \times 10^{-4}$ & $1.01 \times 10^{1}$ \\
\hline & & & & $\mathrm{Pu}-241+\mathrm{D} 30$ & $1.88 \times 10^{-5}$ & $1.89 \times 10^{-5}$ & $9.95 \times 10^{-1}$ \\
\hline & & & & $\mathrm{Pu}-242$ & $1.24 \times 10^{-3}$ & $1.28 \times 10^{-4}$ & 9.69 \\
\hline & & & & $\mathrm{Pu}-244+\mathrm{D} 30$ & 2.23 & 7.73 & $2.88 \times 10^{-1}$ \\
\hline & & & & Am-241 & $4.79 \times 10^{-2}$ & $4.37 \times 10^{-2}$ & 1.10 \\
\hline & & & & Am-243+D30 & 1.08 & $8.95 \times 10^{-1}$ & 1.21 \\
\hline & & & & $\mathrm{Cm}-243$ & $7.26 \times 10^{-1}$ & $5.83 \times 10^{-1}$ & 1.25 \\
\hline & & & & $\mathrm{Cm}-244$ & $1.51 \times 10^{-3}$ & $1.26 \times 10^{-4}$ & $1.20 \times 10^{1}$ \\
\hline & & & & $\mathrm{Cm}-248$ & $6.10 \times 10^{-6}$ & $8.78 \times 10^{-5}$ & $6.95 \times 10^{-2}$ \\
\hline & & & & Cf-252 & $6.32 \times 10^{-5}$ & $1.76 \times 10^{-4}$ & $3.59 \times 10^{-1}$ \\
\hline
\end{tabular}

a Ratio: represents the ratio of previous RESRAD DCFs divided by the FGR-12 DCFs.

b BZ: cases in which both values are zero.

c ZERO: cases in which only RESRAD DCFs are zero.

d INF: cases in which only FGR-12 DCFs are zero.

e +D180: associated radionuclides with half-lives less than 6 months are included.

f +D30: associated radionuclides with half-lives less than 30 days are included. 
- For Ca-45, Sr-89, Tc-99, Pu-238, Pu-240, Pu-242, Cm-244, and Cm-248, $D C F$ s differ by an order of magnitude. Previous RESRAD values are higher in most of these cases.

- For Co-60, Cs-137, Eu-152, Eu-154, Ra-226, Ra-228, Ac-227, Th-228, Th-229, and Np-237, differences in DCFs are between 20 and 50\%.

Table 7 shows the differences in the ratio of the $D C F$ s between the previous RESRAD model and FGR-12 as they increase. "BZ" indicates both values are zero, <20\% means less than 20\% difference in the ratio, 20-100\% means the differences are between 20 and 100\%, >2 represents differences greater than a factor of 2 but less than a factor of 10, and >10 means differences are greater than an order of magnitude.

\subsection{COVER AND DEPTH FACTORS}

Cover and depth factors for the new RESRAD model were compared with the previous model. In these comparisons, the source area was assumed to be of infinite extent, with a density of $1.6 \mathrm{~g} / \mathrm{cm}^{3}$, and only source depths and cover thicknesses were changed. The comparisons were made for Co-60, U-234, U-235, U-238, Mn-54, Al-26, Co-57, and Cs-137. These radionuclides were chosen because of the differences in their average energies.

Table 8 compares the depth factors of the old (version 5.44 and earlier) and new (version 5.60 and later) RESRAD models, and Table 9 gives the depth factor ratio for the old and new models. Figure 3a shows the depth factor as a function of source depth for Co-60, U-234, U-235, U-238, and Mn-54. In this illustration, "n" always represents the results with the new model. Figure $3 \mathrm{~b}$ illustrates the variation of ratio with source depth. Figure 3 shows that no significant difference exists between the old and new RESRAD models for source depths greater than $30 \mathrm{~cm}$; however, thin sources show major differences. The new model gives a higher depth factor for thin sources, which means that the dose calculated with the new model will be higher in these cases.

Table 10 shows the cover-and-depth factor comparisons between the old and new RESRAD models at different source depths. Cover thickness varies from 0.001 to $50 \mathrm{~cm}$. Comparisons are made for Co-60, Mn-54, Al-26, U-234, U-235, U-238, and Co-57. Figure 4a shows the cover factor variations with cover thickness for different source depths of Co-60. To get only the cover factor variations, values were divided by the zero cover thickness for the respective source depths. Figure $4 \mathrm{~b}$ gives the ratio of cover factor for old to new models as a function of cover thickness. Figure 5a shows the cover-and-depth factor variations with cover thickness for source depths of 1, 5, 15, and $50 \mathrm{~cm}$. Figure $5 \mathrm{~b}$ gives the ratio of cover-and-depth factor variations for a Co-60 source. 
TABLE 7 Comparison of the Ratio of the DCFs between the Previous RESRAD Model and FGR-12 for 30-Day Equilibration Radionuclides

\begin{tabular}{|c|c|c|}
\hline Ratio $^{\mathrm{a}}$ & Number & Radionuclides \\
\hline BZ & 4 & $\mathrm{H}-3, \mathrm{~N}-63, \mathrm{Sm}-147, \mathrm{Gd}-152$ \\
\hline$<20 \%$ & 37 & $\begin{array}{l}\text { Na-22, Al-26, K-40, Sc-46, Mn-54, Fe-59, Co-57, Zn-65, Ge-68+D, Se-75, } \\
\text { Sr-85, Zr-95+D, Nb-95, Ru-106+D, Ag-108m+D, Ag-110m+D, Cd-108, } \\
\text { Sn-113+D, Sb-124, Sb-125, Cs-134, Ce-141, Ce-144+D, Eu-155, Gd-153, Ta-182, } \\
\text { Ir-192, Au-195, Pb-210+D, Bi-207, Po-210, Pa-231, U-233, U-235+D, U-238+D, } \\
\text { Pu-241+D, Am-241 }\end{array}$ \\
\hline $20-100 \%$ & 18 & $\begin{array}{l}\text { Co-60, Nb-94, Te-125m, I-125, Cs-137+D, Sm-151, Eu-152,Eu-154, Tl-204, } \\
\text { Ra-226+D, Ra-228+D, Ac-227+D, Th-228+D, Th-229+D, Th-230, Np-237+D, } \\
\text { Am-243+D, Cm-243 }\end{array}$ \\
\hline$>2$ & 11 & $\begin{array}{l}\text { Cl-36, I-129, Pm-147, Th-232, U-232, U-234, U-236, Pu-239, Pu-242, Pu-244+D, } \\
\text { Cf-252 }\end{array}$ \\
\hline$>10$ & 7 & Ca-45, Sr-89, Tc-99, Pu-238, Pu-240, Cm-244, Cm-248 \\
\hline INF & 3 & $\mathrm{Ca}-41, \mathrm{Fe}-55, \mathrm{Ni}-59$ \\
\hline ZERO & 4 & C-14, S-35, Sr-90+D, Cs-135 \\
\hline
\end{tabular}

a Notation: BZ: both zero; INF: cases in which only FGR-12 DCFs are zero; ZERO: cases in which only RESRAD DCFs are zero; $<20 \%$ : less than 20\% difference in ratio; 20-100\%: differences between 20 and $100 \%$; $>2$ : differences greater than a factor of 2 but less than factor of 10; >10: differences greater than a factor of ten.

Table 10 shows that for all radionuclides considered, while the old RESRAD cover factor was independent of source thickness, the new model yields a sharper decrease in cover factor with an increase in cover thickness at small cover. The cover factor comparison shows that the maximum differences occur for a large cover thickness and small source depths. Figure $4 \mathrm{~b}$ shows that the ratio of the old to new models varies from 1 to 3 with an increase in the cover thickness. The cover-anddepth factor comparisons in Figure 5 show that large differences are observed for thin sources without any cover and for sources with very thick covers. For all source depths with a cover between 0.5 and $10 \mathrm{~cm}$, the ratio is between 0.8 and 1.2 .

\subsection{AREA FACTOR}

In the old RESRAD model, a radionuclide-independent area factor was used (Napier et al. 1984). Measurements were made for surface sources of maximum area $1,222 \mathrm{~m}^{2}$ (equivalent to 19.72-m radius). Values at different areas were divided by the maximum value to get the area factor. It was assumed that there was no contribution from regions beyond 19.72-m radius. All the sources used had average energies greater than $100 \mathrm{keV}$. In that study, it was observed that when plotted against area, the exposure rate was parallel for all isotopes, which suggests that the area factor does 
TABLE 8 Comparison of Depth Factors for Old and New RESRAD Models for Co-60, U-234, U-235, U-238, Mn-54, Al-26, Co-57, and Cs-137

\begin{tabular}{|c|c|c|c|c|c|c|c|c|}
\hline \multirow{2}{*}{$\begin{array}{c}\text { Source } \\
\text { Depth }(\mathrm{cm})\end{array}$} & \multicolumn{2}{|c|}{ Co-60 } & \multicolumn{2}{|c|}{ U-234 } & \multicolumn{2}{|c|}{ U-235 } & \multicolumn{2}{|c|}{$\mathrm{U}-238$} \\
\hline & Old & New & Old & New & Old & New & Old & New \\
\hline 1 & $1.06 \times 10^{-1}$ & $1.75 \times 10^{-1}$ & $2.18 \times 10^{-1}$ & $4.70 \times 10^{-1}$ & $2.04 \times 10^{-1}$ & $2.51 \times 10^{-1}$ & $1.21 \times 10^{-1}$ & $2.35 \times 10^{-1}$ \\
\hline 3 & $2.86 \times 10^{-1}$ & $3.65 \times 10^{-1}$ & $5.22 \times 10^{-1}$ & $7.15 \times 10^{-1}$ & $4.96 \times 10^{-1}$ & $5.21 \times 10^{-1}$ & $3.20 \times 10^{-1}$ & $4.47 \times 10^{-1}$ \\
\hline 5 & $4.30 \times 10^{-1}$ & $5.06 \times 10^{-1}$ & $7.08 \times 10^{-1}$ & $8.47 \times 10^{-1}$ & $6.81 \times 10^{-1}$ & $6.92 \times 10^{-1}$ & $4.75 \times 10^{-1}$ & $5.88 \times 10^{-1}$ \\
\hline 10 & $6.75 \times 10^{-1}$ & $7.36 \times 10^{-1}$ & $9.15 \times 10^{-1}$ & $9.68 \times 10^{-1}$ & $8.98 \times 10^{-1}$ & $8.98 \times 10^{-1}$ & $7.24 \times 10^{-1}$ & $8.03 \times 10^{-1}$ \\
\hline 15 & $8.15 \times 10^{-1}$ & $8.59 \times 10^{-1}$ & $9.75 \times 10^{-1}$ & $9.93 \times 10^{-1}$ & $9.68 \times 10^{-1}$ & $9.66 \times 10^{-1}$ & $8.55 \times 10^{-1}$ & $9.05 \times 10^{-1}$ \\
\hline 30 & $9.66 \times 10^{-1}$ & $9.79 \times 10^{-1}$ & $9.99 \times 10^{-1}$ & 1.00 & $9.99 \times 10^{-1}$ & $9.99 \times 10^{-1}$ & $9.79 \times 10^{-1}$ & $9.90 \times 10^{-1}$ \\
\hline 50 & $9.96 \times 10^{-1}$ & $9.98 \times 10^{-1}$ & 1.00 & 1.00 & 1.00 & 1.00 & $9.98 \times 10^{-1}$ & $9.99 \times 10^{-1}$ \\
\hline 100 & $1.0 \underline{0}$ & 1.00 & $1.0 \underline{0}$ & 1.므므. & $1.0 \underline{0}$ & $1.0 \underline{0}$ & 11.00 & -_. \\
\hline \multirow{2}{*}{$\begin{array}{c}\text { Source } \\
\text { Depth }(\mathrm{cm})\end{array}$} & \multicolumn{2}{|c|}{ Mn-54 } & \multicolumn{2}{|c|}{ Al-26 } & \multicolumn{2}{|c|}{ Co-57 } & \multicolumn{2}{|c|}{ Cs-137 } \\
\hline & Old & New & Old & New & Old & New & Old & New \\
\hline 1 & $1.10 \times 10^{-1}$ & $1.93 \times 10^{-1}$ & $9.91 \times 10^{-2}$ & $1.73 \times 10^{-1}$ & $2.01 \times 10^{-1}$ & $2.76 \times 10^{-1}$ & $1.24 \times 10^{-1}$ & $1.95 \times 10^{-1}$ \\
\hline 3 & $2.95 \times 10^{-1}$ & $4.00 \times 10^{-1}$ & $2.69 \times 10^{-1}$ & $3.65 \times 10^{-1}$ & $4.90 \times 10^{-1}$ & $5.69 \times 10^{-1}$ & $3.28 \times 10^{-1}$ & $4.12 \times 10^{-1}$ \\
\hline 5 & $4.42 \times 10^{-1}$ & $5.47 \times 10^{-1}$ & $4.07 \times 10^{-1}$ & $5.07 \times 10^{-1}$ & $6.75 \times 10^{-1}$ & $7.42 \times 10^{-1}$ & $4.84 \times 10^{-1}$ & $5.66 \times 10^{-1}$ \\
\hline 10 & $6.88 \times 10^{-1}$ & $7.76 \times 10^{-1}$ & $6.48 \times 10^{-1}$ & $7.38 \times 10^{-1}$ & $8.94 \times 10^{-1}$ & $9.28 \times 10^{-1}$ & $7.34 \times 10^{-1}$ & $7.97 \times 10^{-1}$ \\
\hline 15 & $8.26 \times 10^{-1}$ & $8.89 \times 10^{-1}$ & $7.91 \times 10^{-1}$ & $8.61 \times 10^{-1}$ & $9.66 \times 10^{-1}$ & $9.80 \times 10^{-1}$ & $8.63 \times 10^{-1}$ & $9.05 \times 10^{-1}$ \\
\hline 30 & $9.70 \times 10^{-1}$ & $9.87 \times 10^{-1}$ & $9.56 \times 10^{-1}$ & $9.79 \times 10^{-1}$ & $9.99 \times 10^{-1}$ & 1.00 & $9.81 \times 10^{-1}$ & $9.90 \times 10^{-1}$ \\
\hline 50 & $9.97 \times 10^{-1}$ & $9.99 \times 10^{-1}$ & $9.95 \times 10^{-1}$ & $9.98 \times 10^{-1}$ & 1.00 & 1.00 & $9.99 \times 10^{-1}$ & 1.00 \\
\hline 100 & 1.00 & 1.00 & 1.00 & 1.00 & 1.00 & 1.00 & 1.00 & 1.00 \\
\hline
\end{tabular}


TABLE 9 Depth Factor Ratio of Old to New RESRAD Models for Different Source Depths for Co-60, U-234, U-235, U-238, Mn-54, Al-26, Co-57, and Cs-137

\begin{tabular}{|c|c|c|c|c|c|c|c|c|}
\hline \multirow{2}{*}{$\begin{array}{c}\text { Source } \\
\text { Depth }(\mathrm{cm})\end{array}$} & \multicolumn{8}{|c|}{ Depth Factor Ratio of Old Model/New Model } \\
\hline & $\mathrm{Co}-60$ & $\mathrm{U}-234$ & $\mathrm{U}-235$ & $\mathrm{U}-238$ & Mn-54 & $\mathrm{Al}-26$ & $\mathrm{Co}-57$ & Cs-137 \\
\hline 1 & $6.08 \times 10^{-1}$ & $4.64 \times 10^{-1}$ & $8.13 \times 10^{-1}$ & $5.15 \times 10^{-1}$ & $5.71 \times 10^{-1}$ & $5.71 \times 10^{-1}$ & $7.27 \times 10^{-1}$ & $6.36 \times 10^{-1}$ \\
\hline 3 & $7.83 \times 10^{-1}$ & $7.30 \times 10^{-1}$ & $9.52 \times 10^{-1}$ & $7.17 \times 10^{-1}$ & $7.39 \times 10^{-1}$ & $7.35 \times 10^{-1}$ & $8.60 \times 10^{-1}$ & $7.98 \times 10^{-1}$ \\
\hline 5 & $8.50 \times 10^{-1}$ & $8.36 \times 10^{-1}$ & $9.84 \times 10^{-1}$ & $8.07 \times 10^{-1}$ & $8.08 \times 10^{-1}$ & $8.00 \times 10^{-1}$ & $9.08 \times 10^{-1}$ & $8.57 \times 10^{-1}$ \\
\hline 10 & $9.17 \times 10^{-1}$ & $9.45 \times 10^{-1}$ & 1.00 & $9.02 \times 10^{-1}$ & $8.87 \times 10^{-1}$ & $8.76 \times 10^{-1}$ & $9.63 \times 10^{-1}$ & $9.22 \times 10^{-1}$ \\
\hline 15 & $9.49 \times 10^{-1}$ & $9.82 \times 10^{-1}$ & 1.00 & $9.44 \times 10^{-1}$ & $9.29 \times 10^{-1}$ & $9.18 \times 10^{-1}$ & $9.85 \times 10^{-1}$ & $9.54 \times 10^{-1}$ \\
\hline 30 & $9.87 \times 10^{-1}$ & $9.99 \times 10^{-1}$ & 1.00 & $9.89 \times 10^{-1}$ & $9.83 \times 10^{-1}$ & $9.76 \times 10^{-1}$ & $9.99 \times 10^{-1}$ & $9.91 \times 10^{-1}$ \\
\hline 50 & $9.98 \times 10^{-1}$ & 1.00 & 1.00 & $9.99 \times 10^{-1}$ & $9.98 \times 10^{-1}$ & $9.96 \times 10^{-1}$ & 1.00 & $9.99 \times 10^{-1}$ \\
\hline 100 & 1.00 & 1.00 & 1.00 & 1.00 & 1.00 & 1.00 & 1.00 & 1.00 \\
\hline
\end{tabular}

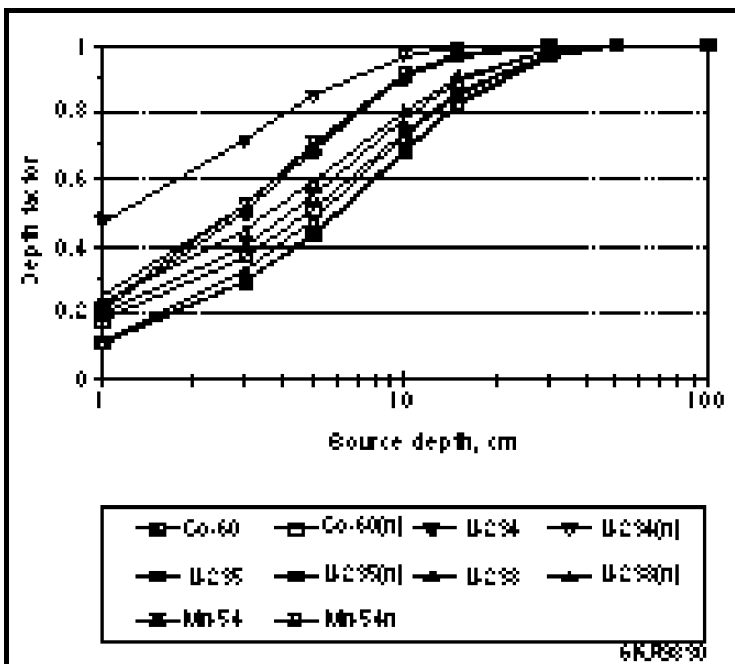

(a) Actual depth for old (solid) and new (open) RESRAD models

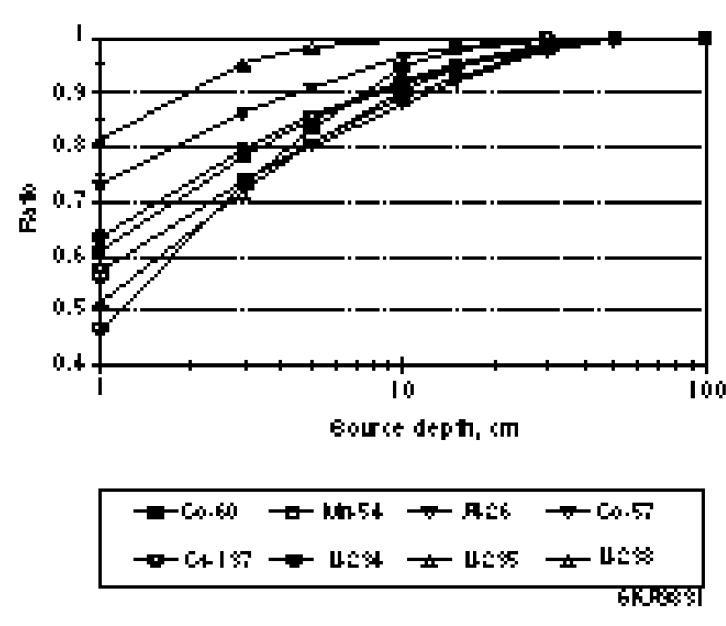

(b) Ratio of depth factor for RESRAD models

FIGURE 3 Depth Factor Comparison as a Function of Depth for a Set of Radionuclides 
TABLE 10 Cover-and-Depth Factor Comparison of Old and New RESRAD Models for Various Radionuclides at Source Depths of 1, 5, 15, and $50 \mathrm{~cm}$

\begin{tabular}{|c|c|c|c|c|c|c|c|c|}
\hline \multirow{3}{*}{$\begin{array}{c}\text { Cover } \\
\text { Thickness }(\mathrm{cm}) \\
\end{array}$} & \multicolumn{8}{|c|}{ Cover-and-Depth Factor by Source Depth } \\
\hline & \multicolumn{2}{|c|}{$1 \mathrm{~cm}$} & \multicolumn{2}{|c|}{$5 \mathrm{~cm}$} & \multicolumn{2}{|c|}{$15 \mathrm{~cm}$} & \multicolumn{2}{|c|}{$50 \mathrm{~cm}$} \\
\hline & Old & New & Old & New & Old & New & Old & New \\
\hline \multicolumn{9}{|l|}{ Co-60 } \\
\hline 0.001 & $1.06 \times 10^{-1}$ & $1.75 \times 10^{-1}$ & $4.30 \times 10^{-1}$ & $5.06 \times 10^{-1}$ & $8.15 \times 10^{-1}$ & $8.59 \times 10^{-1}$ & $9.96 \times 10^{-1}$ & $9.98 \times 10^{-1}$ \\
\hline 0.5 & $1.01 \times 10^{-1}$ & $1.26 \times 10^{-1}$ & $4.07 \times 10^{-1}$ & $4.32 \times 10^{-1}$ & $7.70 \times 10^{-1}$ & $7.63 \times 10^{-1}$ & $9.42 \times 10^{-1}$ & $8.94 \times 10^{-1}$ \\
\hline 1 & $9.50 \times 10^{-2}$ & $1.05 \times 10^{-1}$ & $3.84 \times 10^{-1}$ & $3.90 \times 10^{-1}$ & $7.28 \times 10^{-1}$ & $7.01 \times 10^{-1}$ & $8.90 \times 10^{-1}$ & $8.24 \times 10^{-1}$ \\
\hline 2 & $8.49 \times 10^{-2}$ & $8.59 \times 10^{-2}$ & $3.44 \times 10^{-1}$ & $3.36 \times 10^{-1}$ & $6.51 \times 10^{-1}$ & $6.11 \times 10^{-1}$ & $7.96 \times 10^{-1}$ & $7.19 \times 10^{-1}$ \\
\hline 5 & $6.06 \times 10^{-2}$ & $5.82 \times 10^{-2}$ & $2.45 \times 10^{-1}$ & $2.30 \times 10^{-1}$ & $4.64 \times 10^{-1}$ & $4.19 \times 10^{-1}$ & $5.68 \times 10^{-1}$ & $4.93 \times 10^{-1}$ \\
\hline 10 & $3.45 \times 10^{-2}$ & $3.11 \times 10^{-2}$ & $1.40 \times 10^{-1}$ & $1.23 \times 10^{-1}$ & $2.65 \times 10^{-1}$ & $2.24 \times 10^{-1}$ & $3.23 \times 10^{-1}$ & $2.64 \times 10^{-1}$ \\
\hline 15 & $1.96 \times 10^{-2}$ & $1.66 \times 10^{-2}$ & $7.95 \times 10^{-2}$ & $6.57 \times 10^{-2}$ & $1.51 \times 10^{-1}$ & $1.20 \times 10^{-1}$ & $1.84 \times 10^{-1}$ & $1.41 \times 10^{-1}$ \\
\hline 20 & $1.12 \times 10^{-2}$ & $8.88 \times 10^{-3}$ & $4.53 \times 10^{-2}$ & $3.51 \times 10^{-2}$ & $8.59 \times 10^{-2}$ & $6.39 \times 10^{-2}$ & $1.05 \times 10^{-1}$ & $7.53 \times 10^{-2}$ \\
\hline 25 & $6.39 \times 10^{-3}$ & $4.75 \times 10^{-3}$ & $2.58 \times 10^{-2}$ & $1.88 \times 10^{-2}$ & $4.89 \times 10^{-2}$ & $3.42 \times 10^{-2}$ & $5.98 \times 10^{-2}$ & $4.02 \times 10^{-2}$ \\
\hline 30 & $3.64 \times 10^{-3}$ & $2.54 \times 10^{-3}$ & $1.47 \times 10^{-2}$ & $1.00 \times 10^{-2}$ & $2.78 \times 10^{-2}$ & $1.83 \times 10^{-2}$ & $3.40 \times 10^{-2}$ & $2.15 \times 10^{-2}$ \\
\hline 40 & $1.18 \times 10^{-3}$ & $7.25 \times 10^{-4}$ & $4.78 \times 10^{-3}$ & $2.87 \times 10^{-3}$ & $9.05 \times 10^{-3}$ & $5.22 \times 10^{-3}$ & $1.10 \times 10^{-2}$ & $6.15 \times 10^{-3}$ \\
\hline 50 & $3.84 \times 10^{-4}$ & $2.07 \times 10^{-4}$ & $1.55 \times 10^{-3}$ & $8.19 \times 10^{-4}$ & $2.94 \times 10^{-3}$ & $1.49 \times 10^{-3}$ & $3.60 \times 10^{-3}$ & $1.76 \times 10^{-3}$ \\
\hline \multicolumn{9}{|l|}{ Mn-54 } \\
\hline 0.001 & $1.10 \times 10^{-1}$ & $1.93 \times 10^{-1}$ & $4.42 \times 10^{-1}$ & $5.47 \times 10^{-1}$ & $8.26 \times 10^{-1}$ & $8.89 \times 10^{-1}$ & $9.97 \times 10^{-1}$ & $9.99 \times 10^{-1}$ \\
\hline 0.5 & $1.04 \times 10^{-1}$ & $1.40 \times 10^{-1}$ & $4.17 \times 10^{-1}$ & $4.63 \times 10^{-1}$ & $7.79 \times 10^{-1}$ & $7.82 \times 10^{-1}$ & $9.41 \times 10^{-1}$ & $8.84 \times 10^{-1}$ \\
\hline 1 & $9.79 \times 10^{-2}$ & $1.15 \times 10^{-1}$ & $3.93 \times 10^{-1}$ & $4.14 \times 10^{-1}$ & $7.35 \times 10^{-1}$ & $7.11 \times 10^{-1}$ & $8.87 \times 10^{-1}$ & $8.06 \times 10^{-1}$ \\
\hline 2 & $8.71 \times 10^{-2}$ & $9.22 \times 10^{-2}$ & $3.50 \times 10^{-1}$ & $3.51 \times 10^{-1}$ & $6.54 \times 10^{-1}$ & $6.09 \times 10^{-1}$ & $7.90 \times 10^{-1}$ & $6.92 \times 10^{-1}$ \\
\hline 5 & $6.14 \times 10^{-2}$ & $5.94 \times 10^{-2}$ & $2.47 \times 10^{-1}$ & $2.29 \times 10^{-1}$ & $4.61 \times 10^{-1}$ & $3.98 \times 10^{-1}$ & $5.57 \times 10^{-1}$ & $4.52 \times 10^{-1}$ \\
\hline 10 & $3.42 \times 10^{-2}$ & $2.94 \times 10^{-2}$ & $1.38 \times 10^{-1}$ & $1.13 \times 10^{-1}$ & $2.57 \times 10^{-1}$ & $1.97 \times 10^{-1}$ & $3.11 \times 10^{-1}$ & $2.24 \times 10^{-1}$ \\
\hline 15 & $1.91 \times 10^{-2}$ & $1.45 \times 10^{-2}$ & $7.68 \times 10^{-2}$ & $5.60 \times 10^{-2}$ & $1.44 \times 10^{-1}$ & $9.73 \times 10^{-2}$ & $1.73 \times 10^{-1}$ & $1.11 \times 10^{-1}$ \\
\hline 20 & $1.06 \times 10^{-2}$ & $7.19 \times 10^{-3}$ & $4.29 \times 10^{-2}$ & $2.77 \times 10^{-2}$ & $8.02 \times 10^{-2}$ & $4.81 \times 10^{-2}$ & $9.68 \times 10^{-2}$ & $5.47 \times 10^{-2}$ \\
\hline 25 & $5.97 \times 10^{-3}$ & $3.56 \times 10^{-3}$ & $2.39 \times 10^{-2}$ & $1.37 \times 10^{-2}$ & $4.47 \times 10^{-2}$ & $2.38 \times 10^{-2}$ & $5.40 \times 10^{-2}$ & $2.71 \times 10^{-2}$ \\
\hline 30 & $3.33 \times 10^{-3}$ & $1.76 \times 10^{-3}$ & $1.33 \times 10^{-2}$ & $6.77 \times 10^{-3}$ & $2.50 \times 10^{-2}$ & $1.18 \times 10^{-2}$ & $3.01 \times 10^{-2}$ & $1.34 \times 10^{-2}$ \\
\hline 40 & $1.04 \times 10^{-3}$ & $4.30 \times 10^{-4}$ & $4.17 \times 10^{-3}$ & $1.68 \times 10^{-3}$ & $7.79 \times 10^{-3}$ & $2.88 \times 10^{-3}$ & $9.41 \times 10^{-3}$ & $3.27 \times 10^{-3}$ \\
\hline 50 & $3.24 \times 10^{-4}$ & $1.05 \times 10^{-4}$ & $1.30 \times 10^{-3}$ & $4.05 \times 10^{-4}$ & $2.43 \times 10^{-3}$ & $7.05 \times 10^{-4}$ & $2.93 \times 10^{-3}$ & $8.01 \times 10^{-4}$ \\
\hline
\end{tabular}


TABLE 10 (Cont.)

\begin{tabular}{|c|c|c|c|c|c|c|c|c|}
\hline \multirow{3}{*}{$\begin{array}{c}\text { Cover } \\
\text { Thickness }(\mathrm{cm})\end{array}$} & \multicolumn{8}{|c|}{ Cover-and-Depth Factor by Source Depth } \\
\hline & \multicolumn{2}{|c|}{$1 \mathrm{~cm}$} & \multicolumn{2}{|c|}{$5 \mathrm{~cm}$} & \multicolumn{2}{|c|}{$15 \mathrm{~cm}$} & \multicolumn{2}{|c|}{$50 \mathrm{~cm}$} \\
\hline & Old & New & Old & New & Old & New & Old & New \\
\hline \multicolumn{9}{|l|}{ Al-26 } \\
\hline 0.001 & $9.91 \times 10^{-2}$ & $1.73 \times 10^{-1}$ & $4.07 \times 10^{-1}$ & $5.07 \times 10^{-1}$ & $7.91 \times 10^{-1}$ & $8.61 \times 10^{-1}$ & $9.95 \times 10^{-1}$ & $9.98 \times 10^{-1}$ \\
\hline 0.5 & $9.41 \times 10^{-2}$ & $1.26 \times 10^{-1}$ & $3.86 \times 10^{-1}$ & $4.34 \times 10^{-1}$ & $7.51 \times 10^{-1}$ & $7.66 \times 10^{-1}$ & $9.44 \times 10^{-1}$ & $8.96 \times 10^{-1}$ \\
\hline 1 & $8.93 \times 10^{-2}$ & $1.05 \times 10^{-1}$ & $3.66 \times 10^{-1}$ & $3.92 \times 10^{-1}$ & $7.13 \times 10^{-1}$ & $7.04 \times 10^{-1}$ & $8.96 \times 10^{-1}$ & $8.26 \times 10^{-1}$ \\
\hline 2 & $8.04 \times 10^{-2}$ & $8.66 \times 10^{-2}$ & $3.30 \times 10^{-1}$ & $3.39 \times 10^{-1}$ & $6.42 \times 10^{-1}$ & $6.14 \times 10^{-1}$ & $8.07 \times 10^{-1}$ & $7.21 \times 10^{-1}$ \\
\hline 5 & $5.88 \times 10^{-2}$ & $5.86 \times 10^{-2}$ & $2.41 \times 10^{-1}$ & $2.31 \times 10^{-1}$ & $4.69 \times 10^{-1}$ & $4.19 \times 10^{-1}$ & $5.90 \times 10^{-1}$ & $4.92 \times 10^{-1}$ \\
\hline 10 & $3.48 \times 10^{-2}$ & $3.11 \times 10^{-2}$ & $1.43 \times 10^{-1}$ & $1.23 \times 10^{-1}$ & $2.78 \times 10^{-1}$ & $2.23 \times 10^{-1}$ & $3.50 \times 10^{-1}$ & $2.61 \times 10^{-1}$ \\
\hline 15 & $2.07 \times 10^{-2}$ & $1.66 \times 10^{-2}$ & $8.49 \times 10^{-2}$ & $6.53 \times 10^{-2}$ & $1.65 \times 10^{-1}$ & $1.18 \times 10^{-1}$ & $2.08 \times 10^{-1}$ & $1.39 \times 10^{-1}$ \\
\hline 20 & $1.22 \times 10^{-2}$ & $8.80 \times 10^{-3}$ & $5.03 \times 10^{-2}$ & $3.47 \times 10^{-2}$ & $9.79 \times 10^{-2}$ & $6.30 \times 10^{-2}$ & $1.23 \times 10^{-1}$ & $7.39 \times 10^{-2}$ \\
\hline 25 & $7.29 \times 10^{-3}$ & $4.68 \times 10^{-3}$ & $2.98 \times 10^{-2}$ & $1.84 \times 10^{-2}$ & $5.81 \times 10^{-2}$ & $3.35 \times 10^{-2}$ & $7.30 \times 10^{-2}$ & $3.93 \times 10^{-2}$ \\
\hline 30 & $4.32 \times 10^{-3}$ & $2.49 \times 10^{-3}$ & $1.77 \times 10^{-2}$ & $9.80 \times 10^{-3}$ & $3.44 \times 10^{-2}$ & $1.78 \times 10^{-2}$ & $4.33 \times 10^{-2}$ & $2.09 \times 10^{-2}$ \\
\hline 40 & $1.52 \times 10^{-3}$ & $7.02 \times 10^{-4}$ & $6.24 \times 10^{-3}$ & $2.77 \times 10^{-3}$ & $1.21 \times 10^{-2}$ & $5.02 \times 10^{-3}$ & $1.52 \times 10^{-2}$ & $5.90 \times 10^{-3}$ \\
\hline 50 & $5.35 \times 10^{-4}$ & $1.98 \times 10^{-4}$ & $2.20 \times 10^{-3}$ & $7.83 \times 10^{-4}$ & $4.27 \times 10^{-3}$ & $1.42 \times 10^{-3}$ & $5.37 \times 10^{-3}$ & $1.67 \times 10^{-3}$ \\
\hline \multicolumn{9}{|l|}{ U-234 } \\
\hline 0.001 & $2.18 \times 10^{-1}$ & $4.67 \times 10^{-1}$ & $7.08 \times 10^{-1}$ & $8.44 \times 10^{-1}$ & $9.75 \times 10^{-1}$ & $9.90 \times 10^{-1}$ & 1.00 & $9.97 \times 10^{-1}$ \\
\hline 0.5 & $1.93 \times 10^{-1}$ & $1.66 \times 10^{-1}$ & $6.26 \times 10^{-1}$ & $4.89 \times 10^{-1}$ & $8.62 \times 10^{-1}$ & $6.14 \times 10^{-1}$ & $8.84 \times 10^{-1}$ & $6.20 \times 10^{-1}$ \\
\hline 1 & $1.71 \times 10^{-1}$ & $1.41 \times 10^{-1}$ & $5.53 \times 10^{-1}$ & $4.18 \times 10^{-1}$ & $7.63 \times 10^{-1}$ & $5.25 \times 10^{-1}$ & $7.82 \times 10^{-1}$ & $5.30 \times 10^{-1}$ \\
\hline 2 & $1.33 \times 10^{-1}$ & $1.04 \times 10^{-1}$ & $4.33 \times 10^{-1}$ & $3.06 \times 10^{-1}$ & $5.96 \times 10^{-1}$ & $3.85 \times 10^{-1}$ & $6.12 \times 10^{-1}$ & $3.89 \times 10^{-1}$ \\
\hline 5 & $6.37 \times 10^{-2}$ & $4.09 \times 10^{-2}$ & $2.07 \times 10^{-1}$ & $1.21 \times 10^{-1}$ & $2.85 \times 10^{-1}$ & $1.52 \times 10^{-1}$ & $2.92 \times 10^{-1}$ & $1.53 \times 10^{-1}$ \\
\hline 10 & $1.86 \times 10^{-2}$ & $8.66 \times 10^{-3}$ & $6.05 \times 10^{-2}$ & $2.56 \times 10^{-2}$ & $8.34 \times 10^{-2}$ & $3.21 \times 10^{-2}$ & $8.55 \times 10^{-2}$ & $3.24 \times 10^{-2}$ \\
\hline 15 & $5.45 \times 10^{-3}$ & $1.83 \times 10^{-3}$ & $1.77 \times 10^{-2}$ & $5.42 \times 10^{-3}$ & $2.44 \times 10^{-2}$ & $6.81 \times 10^{-3}$ & $2.50 \times 10^{-2}$ & $6.87 \times 10^{-3}$ \\
\hline 20 & $1.59 \times 10^{-3}$ & $3.88 \times 10^{-4}$ & $5.17 \times 10^{-3}$ & $1.15 \times 10^{-3}$ & $7.13 \times 10^{-3}$ & $1.44 \times 10^{-3}$ & $7.31 \times 10^{-3}$ & $1.46 \times 10^{-3}$ \\
\hline 25 & $4.66 \times 10^{-4}$ & $8.23 \times 10^{-5}$ & $1.51 \times 10^{-3}$ & $2.43 \times 10^{-4}$ & $2.08 \times 10^{-3}$ & $3.05 \times 10^{-4}$ & $2.14 \times 10^{-3}$ & $3.08 \times 10^{-4}$ \\
\hline 30 & $1.36 \times 10^{-4}$ & $1.74 \times 10^{-5}$ & $4.42 \times 10^{-4}$ & $5.15 \times 10^{-5}$ & $6.09 \times 10^{-4}$ & $6.47 \times 10^{-5}$ & $6.25 \times 10^{-4}$ & $6.53 \times 10^{-5}$ \\
\hline
\end{tabular}


TABLE 10 (Cont.)

\begin{tabular}{|c|c|c|c|c|c|c|c|c|}
\hline \multirow{3}{*}{$\begin{array}{c}\text { Cover } \\
\text { Thickness }(\mathrm{cm})\end{array}$} & \multicolumn{8}{|c|}{ Cover-and-Depth Factor by Source Depth } \\
\hline & \multicolumn{2}{|c|}{$1 \mathrm{~cm}$} & \multicolumn{2}{|c|}{$5 \mathrm{~cm}$} & \multicolumn{2}{|c|}{$15 \mathrm{~cm}$} & \multicolumn{2}{|c|}{$50 \mathrm{~cm}$} \\
\hline & Old & New & Old & New & Old & New & Old & New \\
\hline \multicolumn{9}{|l|}{ U-235 } \\
\hline 0.001 & $2.04 \times 10^{-1}$ & $2.51 \times 10^{-1}$ & $6.81 \times 10^{-1}$ & $6.92 \times 10^{-1}$ & $9.67 \times 10^{-1}$ & $9.66 \times 10^{-1}$ & 1.00 & 1.00 \\
\hline 0.5 & $1.82 \times 10^{-1}$ & $1.81 \times 10^{-1}$ & $6.07 \times 10^{-1}$ & $5.73 \times 10^{-1}$ & $8.63 \times 10^{-1}$ & $8.13 \times 10^{-1}$ & $8.92 \times 10^{-1}$ & $8.49 \times 10^{-1}$ \\
\hline 1 & $1.62 \times 10^{-1}$ & $1.51 \times 10^{-1}$ & $5.42 \times 10^{-1}$ & $5.02 \times 10^{-1}$ & $7.70 \times 10^{-1}$ & $7.22 \times 10^{-1}$ & $7.96 \times 10^{-1}$ & $7.49 \times 10^{-1}$ \\
\hline 2 & $1.29 \times 10^{-1}$ & $1.19 \times 10^{-1}$ & $4.31 \times 10^{-1}$ & $4.00 \times 10^{-1}$ & $6.13 \times 10^{-1}$ & $5.76 \times 10^{-1}$ & $6.33 \times 10^{-1}$ & $5.98 \times 10^{-1}$ \\
\hline 5 & $6.52 \times 10^{-2}$ & $6.10 \times 10^{-2}$ & $2.17 \times 10^{-1}$ & $2.06 \times 10^{-1}$ & $3.09 \times 10^{-1}$ & $2.97 \times 10^{-1}$ & $3.19 \times 10^{-1}$ & $3.08 \times 10^{-1}$ \\
\hline 10 & $2.08 \times 10^{-2}$ & $2.02 \times 10^{-2}$ & $6.93 \times 10^{-2}$ & $6.83 \times 10^{-2}$ & $9.85 \times 10^{-2}$ & $9.84 \times 10^{-2}$ & $1.02 \times 10^{-1}$ & $1.02 \times 10^{-1}$ \\
\hline 15 & $6.64 \times 10^{-3}$ & $6.71 \times 10^{-3}$ & $2.21 \times 10^{-2}$ & $2.26 \times 10^{-2}$ & $3.14 \times 10^{-2}$ & $3.26 \times 10^{-2}$ & $3.25 \times 10^{-2}$ & $3.39 \times 10^{-2}$ \\
\hline 20 & $2.12 \times 10^{-3}$ & $2.22 \times 10^{-3}$ & $7.06 \times 10^{-3}$ & $7.50 \times 10^{-3}$ & $1.00 \times 10^{-2}$ & $1.08 \times 10^{-2}$ & $1.04 \times 10^{-2}$ & $1.12 \times 10^{-2}$ \\
\hline 25 & $6.76 \times 10^{-4}$ & $7.37 \times 10^{-4}$ & $2.25 \times 10^{-3}$ & $2.49 \times 10^{-3}$ & $3.20 \times 10^{-3}$ & $3.59 \times 10^{-3}$ & $3.31 \times 10^{-3}$ & $3.72 \times 10^{-3}$ \\
\hline 30 & $2.16 \times 10^{-4}$ & $2.44 \times 10^{-4}$ & $7.19 \times 10^{-4}$ & $8.25 \times 10^{-4}$ & $1.02 \times 10^{-3}$ & $1.19 \times 10^{-3}$ & $1.06 \times 10^{-3}$ & $1.23 \times 10^{-3}$ \\
\hline 40 & $2.20 \times 10^{-5}$ & $2.69 \times 10^{-5}$ & $7.33 \times 10^{-5}$ & $9.07 \times 10^{-5}$ & $1.04 \times 10^{-4}$ & $1.31 \times 10^{-4}$ & $1.08 \times 10^{-4}$ & $1.36 \times 10^{-4}$ \\
\hline 50 & $2.24 \times 10^{-6}$ & $2.95 \times 10^{-6}$ & $7.46 \times 10^{-6}$ & $9.97 \times 10^{-6}$ & $1.06 \times 10^{-5}$ & $1.44 \times 10^{-5}$ & $1.10 \times 10^{-5}$ & $1.49 \times 10^{-5}$ \\
\hline \multicolumn{9}{|l|}{ U-238 } \\
\hline 0.001 & $1.21 \times 10^{-1}$ & $2.34 \times 10^{-1}$ & $4.80 \times 10^{-1}$ & $5.88 \times 10^{-1}$ & $8.55 \times 10^{-1}$ & $9.05 \times 10^{-1}$ & $9.98 \times 10^{-1}$ & $9.99 \times 10^{-1}$ \\
\hline 0.5 & $1.13 \times 10^{-1}$ & $1.57 \times 10^{-1}$ & $4.45 \times 10^{-1}$ & $4.74 \times 10^{-1}$ & $8.02 \times 10^{-1}$ & $7.68 \times 10^{-1}$ & $9.36 \times 10^{-1}$ & $8.56 \times 10^{-1}$ \\
\hline 1 & $1.06 \times 10^{-1}$ & $1.21 \times 10^{-1}$ & $4.17 \times 10^{-1}$ & $4.10 \times 10^{-1}$ & $7.52 \times 10^{-1}$ & $6.84 \times 10^{-1}$ & $8.78 \times 10^{-1}$ & $7.65 \times 10^{-1}$ \\
\hline 2 & $9.34 \times 10^{-2}$ & $9.09 \times 10^{-2}$ & $3.67 \times 10^{-1}$ & $3.37 \times 10^{-1}$ & $6.61 \times 10^{-1}$ & $5.74 \times 10^{-1}$ & $7.72 \times 10^{-1}$ & $6.44 \times 10^{-1}$ \\
\hline 5 & $6.35 \times 10^{-2}$ & $5.63 \times 10^{-2}$ & $2.49 \times 10^{-1}$ & $2.14 \times 10^{-1}$ & $4.49 \times 10^{-1}$ & $3.67 \times 10^{-1}$ & $5.25 \times 10^{-1}$ & $4.12 \times 10^{-1}$ \\
\hline 10 & $3.33 \times 10^{-2}$ & $2.70 \times 10^{-2}$ & $1.31 \times 10^{-1}$ & $1.03 \times 10^{-1}$ & $2.36 \times 10^{-1}$ & $1.76 \times 10^{-1}$ & $2.76 \times 10^{-1}$ & $1.97 \times 10^{-1}$ \\
\hline 15 & $1.75 \times 10^{-2}$ & $1.29 \times 10^{-2}$ & $6.88 \times 10^{-2}$ & $4.93 \times 10^{-2}$ & $1.24 \times 10^{-1}$ & $8.42 \times 10^{-2}$ & $1.45 \times 10^{-1}$ & $9.46 \times 10^{-2}$ \\
\hline 20 & $9.20 \times 10^{-3}$ & $6.21 \times 10^{-3}$ & $3.62 \times 10^{-2}$ & $2.36 \times 10^{-2}$ & $6.51 \times 10^{-2}$ & $4.04 \times 10^{-2}$ & $7.61 \times 10^{-2}$ & $4.54 \times 10^{-2}$ \\
\hline 25 & $4.83 \times 10^{-3}$ & $2.98 \times 10^{-3}$ & $1.90 \times 10^{-2}$ & $1.13 \times 10^{-2}$ & $3.42 \times 10^{-2}$ & $1.94 \times 10^{-2}$ & $4.00 \times 10^{-2}$ & $2.17 \times 10^{-2}$ \\
\hline 30 & $2.54 \times 10^{-3}$ & $1.43 \times 10^{-3}$ & $1.00 \times 10^{-2}$ & $5.43 \times 10^{-3}$ & $1.80 \times 10^{-2}$ & $9.28 \times 10^{-3}$ & $2.10 \times 10^{-2}$ & $1.04 \times 10^{-2}$ \\
\hline 40 & $7.01 \times 10^{-4}$ & $3.28 \times 10^{-4}$ & $2.75 \times 10^{-3}$ & $1.25 \times 10^{-3}$ & $4.96 \times 10^{-3}$ & $2.13 \times 10^{-3}$ & $5.79 \times 10^{-3}$ & $2.40 \times 10^{-3}$ \\
\hline 50 & $1.94 \times 10^{-4}$ & $7.53 \times 10^{-5}$ & $7.60 \times 10^{-4}$ & $2.87 \times 10^{-4}$ & $1.37 \times 10^{-4}$ & $4.90 \times 10^{-4}$ & $1.60 \times 10^{-3}$ & $5.51 \times 10^{-4}$ \\
\hline
\end{tabular}


TABLE 10 (Cont.)

\begin{tabular}{|c|c|c|c|c|c|c|c|c|}
\hline \multirow{3}{*}{$\begin{array}{c}\text { Cover } \\
\text { Thickness }(\mathrm{cm}) \\
\end{array}$} & \multicolumn{8}{|c|}{ Cover-and-Depth Factor by Source Depth } \\
\hline & \multicolumn{2}{|c|}{$1 \mathrm{~cm}$} & \multicolumn{2}{|c|}{$5 \mathrm{~cm}$} & \multicolumn{2}{|c|}{$15 \mathrm{~cm}$} & \multicolumn{2}{|c|}{$50 \mathrm{~cm}$} \\
\hline & Old & New & Old & New & Old & New & Old & New \\
\hline \multicolumn{9}{|l|}{ Co-57 } \\
\hline 0.001 & $2.01 \times 10^{-1}$ & $2.76 \times 10^{-1}$ & $6.74 \times 10^{-1}$ & $7.42 \times 10^{-1}$ & $9.65 \times 10^{-1}$ & $9.80 \times 10^{-1}$ & 1.00 & 1.00 \\
\hline 0.5 & $1.80 \times 10^{-1}$ & $2.02 \times 10^{-1}$ & $6.03 \times 10^{-1}$ & $6.09 \times 10^{-1}$ & $8.63 \times 10^{-1}$ & $8.19 \times 10^{-1}$ & $8.94 \times 10^{-1}$ & $8.36 \times 10^{-1}$ \\
\hline 1 & $1.61 \times 10^{-1}$ & $1.67 \times 10^{-1}$ & $5.39 \times 10^{-1}$ & $5.24 \times 10^{-1}$ & $7.71 \times 10^{-1}$ & $7.09 \times 10^{-1}$ & $7.99 \times 10^{-1}$ & $7.24 \times 10^{-1}$ \\
\hline 2 & $1.28 \times 10^{-1}$ & $1.26 \times 10^{-1}$ & $4.31 \times 10^{-1}$ & $4.02 \times 10^{-1}$ & $6.16 \times 10^{-1}$ & $5.45 \times 10^{-1}$ & $6.38 \times 10^{-1}$ & $5.57 \times 10^{-1}$ \\
\hline 5 & $6.54 \times 10^{-2}$ & $5.83 \times 10^{-2}$ & $2.20 \times 10^{-1}$ & $1.87 \times 10^{-1}$ & $3.14 \times 10^{-1}$ & $2.53 \times 10^{-1}$ & $3.26 \times 10^{-1}$ & $2.58 \times 10^{-1}$ \\
\hline 10 & $2.13 \times 10^{-2}$ & $1.62 \times 10^{-2}$ & $7.14 \times 10^{-2}$ & $5.19 \times 10^{-2}$ & $1.02 \times 10^{-1}$ & $7.03 \times 10^{-2}$ & $1.06 \times 10^{-1}$ & $7.18 \times 10^{-2}$ \\
\hline 15 & $6.93 \times 10^{-3}$ & $4.51 \times 10^{-3}$ & $2.32 \times 10^{-2}$ & $1.44 \times 10^{-2}$ & $3.33 \times 10^{-2}$ & $1.95 \times 10^{-2}$ & $3.45 \times 10^{-2}$ & $2.00 \times 10^{-2}$ \\
\hline 20 & $2.26 \times 10^{-3}$ & $1.25 \times 10^{-3}$ & $7.57 \times 10^{-3}$ & $4.01 \times 10^{-3}$ & $1.08 \times 10^{-2}$ & $5.43 \times 10^{-3}$ & $1.12 \times 10^{-2}$ & $5.55 \times 10^{-3}$ \\
\hline 25 & $7.35 \times 10^{-4}$ & $3.49 \times 10^{-4}$ & $2.47 \times 10^{-3}$ & $1.11 \times 10^{-3}$ & $3.53 \times 10^{-3}$ & $1.51 \times 10^{-3}$ & $3.66 \times 10^{-3}$ & $1.54 \times 10^{-3}$ \\
\hline 30 & $2.39 \times 10^{-4}$ & $9.69 \times 10^{-5}$ & $8.03 \times 10^{-4}$ & $3.10 \times 10^{-4}$ & $1.15 \times 10^{-3}$ & $4.20 \times 10^{-4}$ & $1.19 \times 10^{-3}$ & $4.29 \times 10^{-4}$ \\
\hline
\end{tabular}




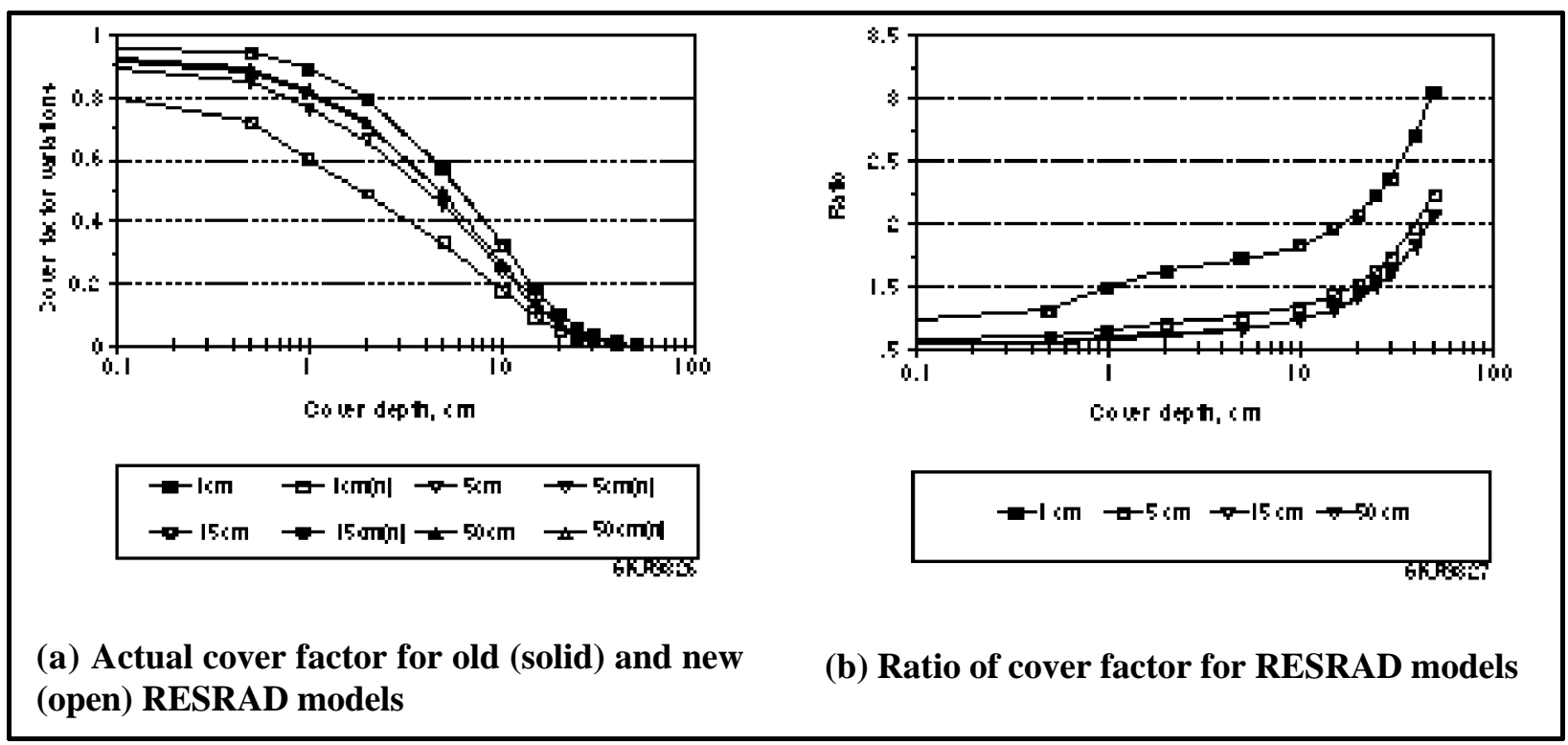

FIGURE 4 Cover Factor Comparison as a Function of Cover Depth for a Set of Co-60-Contaminated Source Depths

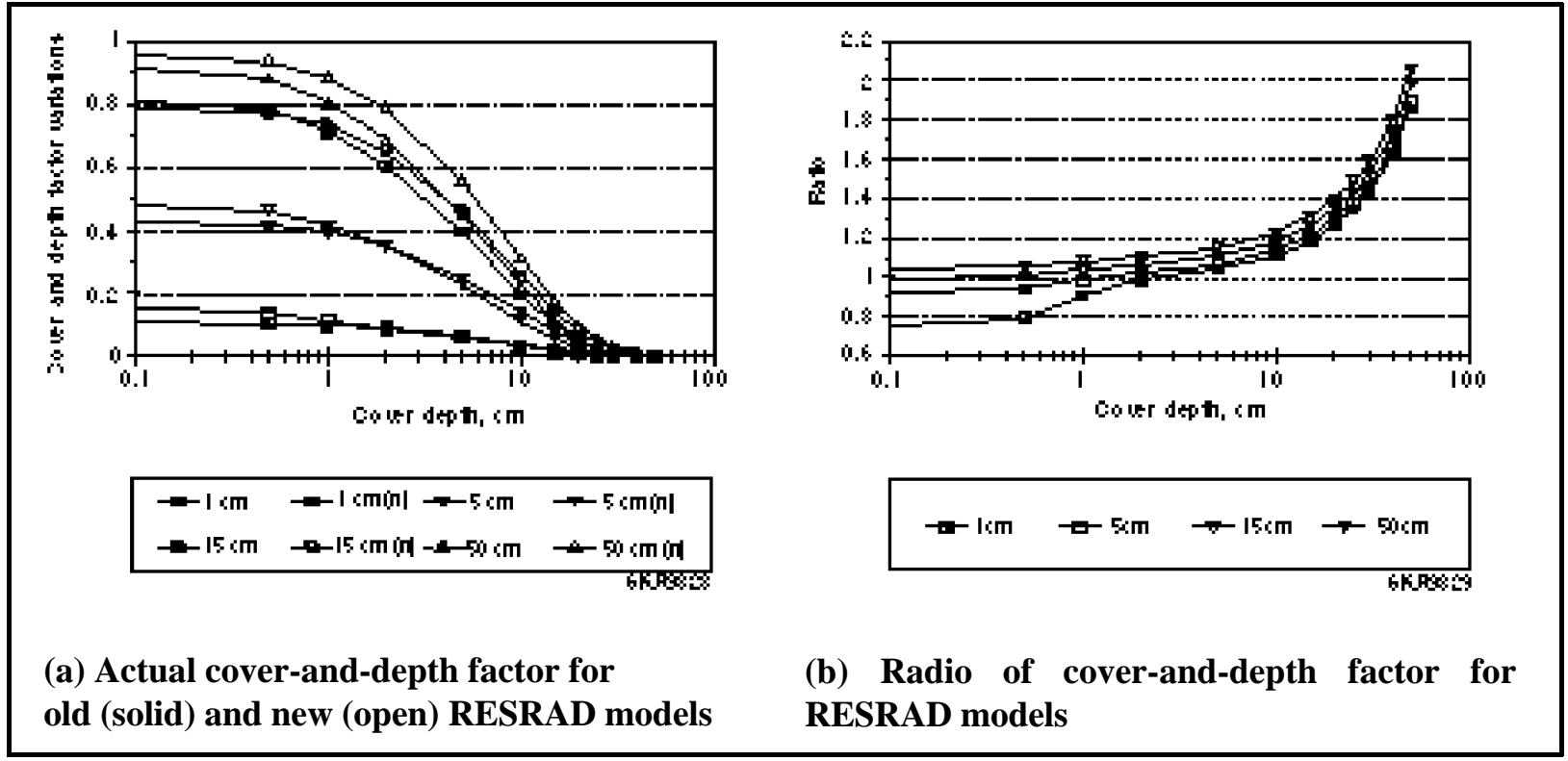

FIGURE 5 Cover-and-Depth Factor Comparison as a Function of Cover Depth for a Set of Co-60Contaminated Source Depths 
not depend on energy. Therefore, a uniform correction was applied for all energies. The area factor correction curve was approximated as the sum of four line segments:

$$
\begin{aligned}
& y=0.016 A \text { for } 0<A<25 \mathrm{~m}^{2}, \\
& y=0.35+0.002 A \text { for } 25<A<100 \mathrm{~m}^{2}, \\
& y=0.48+0.00065 A \text { for } 100<A<500 \mathrm{~m}^{2}, \\
& y=0.67+0.00027 A \text { for } 500<A<1,222 \mathrm{~m}^{2}, \text { and } \\
& y=1 \text { for } A \$ 1,222 \mathrm{~m}^{2} .
\end{aligned}
$$

The results from comparison of the old and new methods are discussed here. The area factor is plotted against the source radius for different energies and is calculated for different source depths. Table 11 gives the area factor, $F_{A}(E i)$, for different source depths. Sources are assumed to be of different energies $(10,30,60,100,300,600,1,000,3,000,6,000$, and 10,000 keV), and the source radius varies from 0.56 to $1,000 \mathrm{~m}$. Different columns give area factors for different energies. Different rows give the area factors at different source radii. Figure 6 shows the area factor variations for different source depths $(0.1,1.0,10.0$, and $100 \mathrm{~cm})$. Each curve in the figures represents the variation with source radius for a particular energy.

As shown in Figure 6, the area factor increases with source radius and approaches unity for radii greater than $50 \mathrm{~m}$. The area factor is always larger in the new model for small source radii for all energies. For the 100-cm-thick source, the area factor is larger up to a source radius of $13 \mathrm{~m}$; for the 1-cm-thick source, it is larger up to a 2-m source radius. The area factor decreases with increasing energy, with sharp decreases when the energy changes from 10 to $60 \mathrm{keV}$, and varies slightly with higher energy. The area factor also increases with increasing source depth. Comparisons show that the area factor of sources with depths greater than $10 \mathrm{~cm}$ are always greater in the new model. For very thin sources $(0.1 \mathrm{~cm}$ depth), the old RESRAD model values are higher except for energies under $30 \mathrm{keV}$, for which the new model gives higher values. Values compare reasonably well in the two models at a source depth of $1 \mathrm{~cm}$ for energies above $30 \mathrm{keV}$.

Tables 12 through 14 show the variation of area factor with cover thickness. Computations are made for three energy levels $(10,100$, and 1,000 keV) and four source depths $(0.1,1,10$, and $100 \mathrm{~cm}$ ). Source radius varies from 0.56 to $1,000 \mathrm{~m}$, and cover thickness varies from 0 to $50 \mathrm{~cm}$ for each set.

Table 12 shows the variation of the area factor with cover material at $10 \mathrm{keV}$. The area factor increases with the source radius for all source depths when there is no cover. With some cover (greater than $0.5 \mathrm{~cm}$ ), the area factor becomes unity and is independent of source dimension. 
TABLE 11 Area Factors for Source Depths of 0.1, 1, 10, and $100 \mathrm{~cm}$ at Different Energies

\begin{tabular}{|c|c|c|c|c|c|c|c|c|c|c|c|}
\hline \multirow[b]{2}{*}{$\begin{array}{c}\text { Radius } \\
(\mathrm{m})\end{array}$} & \multicolumn{11}{|c|}{ Area Factor, $F_{A}$, by Energy Level } \\
\hline & $10 \mathrm{keV}$ & $30 \mathrm{keV}$ & $60 \mathrm{keV}$ & $100 \mathrm{keV}$ & $300 \mathrm{keV}$ & $600 \mathrm{keV}$ & $1 \mathrm{MeV}$ & $3 \mathrm{MeV}$ & $6 \mathrm{MeV}$ & $10 \mathrm{MeV}$ & $\begin{array}{c}\text { Old } \\
\text { Model }\end{array}$ \\
\hline \multicolumn{12}{|c|}{ Source Depth $=0.1 \mathrm{~cm}$} \\
\hline 0.56 & 0.246 & 0.0527 & 0.0234 & 0.0218 & 0.0265 & 0.0275 & 0.0278 & 0.0266 & 0.0259 & 0.0257 & 0.0160 \\
\hline 1 & 0.528 & 0.134 & 0.0599 & 0.0556 & 0.0672 & 0.0697 & 0.0705 & 0.0676 & 0.0657 & 0.0652 & 0.0500 \\
\hline 1.5 & 0.742 & 0.229 & 0.103 & 0.0951 & 0.115 & 0.119 & 0.120 & 0.115 & 0.112 & 0.111 & 0.113 \\
\hline 2 & 0.859 & 0.312 & 0.142 & 0.131 & 0.156 & 0.162 & 0.164 & 0.157 & 0.153 & 0.151 & 0.200 \\
\hline 2.8 & 0.944 & 0.421 & 0.196 & 0.178 & 0.212 & 0.219 & 0.222 & 0.212 & 0.207 & 0.205 & 0.400 \\
\hline 3.5 & 0.974 & 0.497 & 0.235 & 0.213 & 0.251 & 0.260 & 0.263 & 0.252 & 0.245 & 0.243 & 0.427 \\
\hline 4.5 & 0.991 & 0.582 & 0.283 & 0.254 & 0.297 & 0.307 & 0.311 & 0.297 & 0.289 & 0.287 & 0.477 \\
\hline 5.6 & 0.997 & 0.654 & 0.328 & 0.292 & 0.339 & 0.349 & 0.353 & 0.338 & 0.329 & 0.326 & 0.550 \\
\hline 7 & 0.999 & 0.723 & 0.377 & 0.333 & 0.381 & 0.392 & 0.397 & 0.380 & 0.369 & 0.366 & 0.580 \\
\hline 9 & 1.00 & 0.792 & 0.436 & 0.381 & 0.431 & 0.442 & 0.447 & 0.428 & 0.416 & 0.412 & 0.645 \\
\hline 11 & 1.00 & 0.841 & 0.485 & 0.421 & 0.470 & 0.482 & 0.486 & 0.466 & 0.453 & 0.449 & 0.727 \\
\hline 13 & 1.00 & 0.876 & 0.528 & 0.457 & 0.504 & 0.515 & 0.519 & 0.497 & 0.483 & 0.479 & 0.800 \\
\hline 15 & 1.00 & 0.902 & 0.566 & 0.488 & 0.532 & 0.543 & 0.547 & 0.524 & 0.510 & 0.505 & 0.860 \\
\hline 17.5 & 1.00 & 0.925 & 0.608 & 0.523 & 0.564 & 0.574 & 0.578 & 0.553 & 0.538 & 0.533 & 0.929 \\
\hline 20 & 1.00 & 0.942 & 0.645 & 0.554 & 0.591 & 0.600 & 0.604 & 0.578 & 0.562 & 0.557 & 1.00 \\
\hline 50 & 1.00 & 0.994 & 0.881 & 0.789 & 0.781 & 0.777 & 0.776 & 0.742 & 0.722 & 0.715 & 1.00 \\
\hline 100 & 1.00 & 1.00 & 0.979 & 0.939 & 0.909 & 0.894 & 0.889 & 0.852 & 0.830 & 0.822 & 1.00 \\
\hline 200 & 1.00 & 1.00 & 1.00 & 0.998 & 0.986 & 0.972 & 0.966 & 0.936 & 0.917 & 0.909 & 1.00 \\
\hline 500 & 1.00 & 1.00 & 1.00 & 1.00 & 1.00 & 1.00 & 0.999 & 0.992 & 0.984 & 0.978 & 1.00 \\
\hline 1000 & 1.00 & 1.00 & 1.00 & 1.00 & 1.믄 & 1.00 & 1.00 & 1.00 & 1.00 & $\underline{0.9} \underline{9} \underline{8}$ & 1.00 \\
\hline \multicolumn{12}{|c|}{ Source Depth $=1 \mathrm{~cm}$} \\
\hline 0.56 & 0.246 & 0.117 & 0.0417 & 0.0319 & 0.0361 & 0.0376 & 0.0378 & 0.0358 & 0.0347 & 0.0345 & 0.0160 \\
\hline 1 & 0.528 & 0.281 & 0.109 & 0.0826 & 0.0921 & 0.0956 & 0.0961 & 0.0908 & 0.0881 & 0.0876 & 0.0500 \\
\hline 1.5 & 0.741 & 0.442 & 0.190 & 0.144 & 0.157 & 0.163 & 0.164 & 0.155 & 0.150 & 0.149 & 0.113 \\
\hline 2 & 0.858 & 0.561 & 0.265 & 0.200 & 0.216 & 0.222 & 0.223 & 0.211 & 0.204 & 0.203 & 0.200 \\
\hline 2.8 & 0.943 & 0.686 & 0.369 & 0.279 & 0.293 & 0.301 & 0.302 & 0.285 & 0.276 & 0.274 & 0.400 \\
\hline 3.5 & 0.973 & 0.755 & 0.444 & 0.338 & 0.349 & 0.357 & 0.357 & 0.337 & 0.327 & 0.325 & 0.427 \\
\hline 4.5 & 0.990 & 0.819 & 0.531 & 0.410 & 0.415 & 0.422 & 0.421 & 0.397 & 0.385 & 0.382 & 0.477 \\
\hline 5.6 & 0.997 & 0.862 & 0.606 & 0.476 & 0.473 & 0.479 & 0.477 & 0.450 & 0.436 & 0.433 & 0.550 \\
\hline 7 & 0.999 & 0.898 & 0.680 & 0.546 & 0.534 & 0.537 & 0.534 & 0.504 & 0.489 & 0.485 & 0.580 \\
\hline 9 & 1.00 & 0.935 & 0.754 & 0.625 & 0.603 & 0.603 & 0.598 & 0.564 & 0.547 & 0.543 & 0.645 \\
\hline 11 & 1.00 & 0.956 & 0.806 & 0.687 & 0.657 & 0.654 & 0.648 & 0.611 & 0.593 & 0.588 & 0.727 \\
\hline 13 & 1.00 & 0.960 & 0.843 & 0.736 & 0.701 & 0.695 & 0.6878 & 0.649 & 0.630 & 0.625 & 0.800 \\
\hline 15 & 1.00 & 0.968 & 0.869 & 0.775 & 0.737 & 0.729 & 0.721 & 0.681 & 0.661 & 0.655 & 0.860 \\
\hline 17.5 & 1.00 & 0.978 & 0.894 & 0.813 & 0.774 & 0.763 & 0.755 & 0.714 & 0.693 & 0.687 & 0.929 \\
\hline 20 & 1.00 & 0.984 & 0.913 & 0.843 & 0.804 & 0.792 & 0.783 & 0.741 & 0.721 & 0.714 & 1.00 \\
\hline 50 & 1.00 & 0.997 & 0.982 & 0.962 & 0.945 & 0.934 & 0.928 & 0.894 & 0.876 & 0.869 & 1.00 \\
\hline 100 & 1.00 & 1.00 & 0.997 & 0.992 & 0.985 & 0.980 & 0.977 & 0.961 & 0.948 & 0.941 & 1.00 \\
\hline 200 & 1.00 & 1.00 & 1.00 & 0.999 & 0.998 & 0.996 & 0.995 & 0.988 & 0.982 & 0.980 & 1.00 \\
\hline 500 & 1.00 & 1.00 & 1.00 & 1.00 & 1.00 & 1.00 & 1.00 & 1.00 & 0.998 & 0.997 & 1.00 \\
\hline 1000 & 1.00 & 1.00 & 1.00 & 1.00 & 1.00 & 1.00 & 1.00 & 1.00 & 1.00 & 1.00 & 1.00 \\
\hline
\end{tabular}


TABLE 11 (Cont.)

\begin{tabular}{|c|c|c|c|c|c|c|c|c|c|c|c|}
\hline \multirow[b]{2}{*}{$\begin{array}{c}\text { Radius } \\
(\mathrm{m})\end{array}$} & \multicolumn{11}{|c|}{ Area Factor, $F_{A}$, by Energy Level } \\
\hline & $10 \mathrm{keV}$ & $30 \mathrm{keV}$ & $60 \mathrm{keV}$ & $100 \mathrm{keV}$ & $300 \mathrm{keV}$ & $600 \mathrm{keV}$ & $1 \mathrm{MeV}$ & $3 \mathrm{MeV}$ & $6 \mathrm{MeV}$ & $10 \mathrm{MeV}$ & $\begin{array}{c}\text { Old } \\
\text { Model }\end{array}$ \\
\hline \multicolumn{12}{|c|}{ Source Depth $=10 \mathrm{~cm}$} \\
\hline 0.56 & 0.246 & 0.133 & 0.117 & 0.0917 & 0.0781 & 0.0749 & 0.072 & 0.0641 & 0.0604 & 0.0595 & 0.0160 \\
\hline 1 & 0.528 & 0.305 & 0.280 & 0.231 & 0.198 & 0.190 & 0.182 & 0.163 & 0.153 & 0.151 & 0.0500 \\
\hline 1.5 & 0.741 & 0.464 & 0.438 & 0.380 & 0.333 & 0.319 & 0.306 & 0.275 & 0.260 & 0.256 & 0.113 \\
\hline 2 & 0.858 & 0.575 & 0.553 & 0.498 & 0.445 & 0.427 & 0.411 & 0.371 & 0.351 & 0.346 & 0.200 \\
\hline 2.8 & 0.943 & 0.687 & 0.671 & 0.628 & 0.577 & 0.555 & 0.537 & 0.489 & 0.465 & 0.458 & 0.400 \\
\hline 3.5 & 0.973 & 0.747 & 0.737 & 0.702 & 0.656 & 0.634 & 0.615 & 0.567 & 0.540 & 0.533 & 0.427 \\
\hline 4.5 & 0.990 & 0.806 & 0.796 & 0.769 & 0.733 & 0.713 & 0.695 & 0.648 & 0.620 & 0.612 & 0.477 \\
\hline 5.6 & 0.997 & 0.847 & 0.838 & 0.816 & 0.787 & 0.770 & 0.754 & 0.712 & 0.684 & 0.676 & 0.550 \\
\hline 7 & 0.999 & 0.868 & 0.873 & 0.860 & 0.835 & 0.819 & 0.806 & 0.768 & 0.743 & 0.735 & 0.580 \\
\hline 9 & 1.00 & 0.894 & 0.902 & 0.894 & 0.875 & 0.860 & 0.849 & 0.820 & 0.800 & 0.792 & 0.645 \\
\hline 11 & 1.00 & 0.910 & 0.920 & 0.916 & 0.900 & 0.887 & 0.879 & 0.854 & 0.835 & 0.831 & 0.727 \\
\hline 13 & 1.00 & 0.922 & 0.934 & 0.930 & 0.918 & 0.905 & 0.899 & 0.881 & 0.864 & 0.859 & 0.800 \\
\hline 15 & 1.00 & 0.931 & 0.944 & 0.941 & 0.931 & 0.918 & 0.913 & 0.899 & 0.884 & 0.877 & 0.860 \\
\hline 17.5 & 1.00 & 0.941 & 0.946 & 0.951 & 0.940 & 0.932 & 0.925 & 0.915 & 0.901 & 0.896 & 0.929 \\
\hline 20 & 1.00 & 0.949 & 0.949 & 0.958 & 0.948 & 0.941 & 0.935 & 0.927 & 0.914 & 0.909 & 1.00 \\
\hline 50 & 1.00 & 0.990 & 0.973 & 0.979 & 0.981 & 0.979 & 0.976 & 0.974 & 0.970 & 0.968 & 1.00 \\
\hline 100 & 1.00 & 0.999 & 0.991 & 0.990 & 0.989 & 0.988 & 0.988 & 0.987 & 0.986 & 0.985 & 1.00 \\
\hline 200 & 1.00 & 1.00 & 1.00 & 0.998 & 0.996 & 0.995 & 0.995 & 0.994 & 0.993 & 0.992 & 1.00 \\
\hline 500 & 1.00 & 1.00 & 1.00 & 1.00 & 1.00 & 1.00 & 0.999 & 0.999 & 0.998 & 0.998 & 1.00 \\
\hline 1000 & -1.00 - & 1.00 & -1.00 - & -1.00 & -1.00 & $-\underline{1.00}$ & $-\underline{1}-\underline{0}-\underline{0}$ & 1.00 & - 1.00 & 1.00 & 1.00 \\
\hline \multicolumn{12}{|c|}{ Source Depth $=100 \mathrm{~cm}$} \\
\hline 0.56 & 0.246 & 0.133 & 0.121 & 0.114 & 0.106 & 0.100 & 0.0960 & 0.0860 & 0.0820 & 0.0800 & 0.0160 \\
\hline 1 & 0.528 & 0.305 & 0.286 & 0.274 & 0.258 & 0.246 & 0.236 & 0.215 & 0.208 & 0.203 & 0.0500 \\
\hline 1.5 & 0.741 & 0.464 & 0.443 & 0.432 & 0.411 & 0.393 & 0.380 & 0.353 & 0.345 & 0.34 & 0.113 \\
\hline 2 & 0.858 & 0.575 & 0.555 & 0.546 & 0.524 & 0.503 & 0.487 & 0.459 & 0.454 & 0.448 & 0.200 \\
\hline 2.8 & 0.943 & 0.687 & 0.670 & 0.664 & 0.641 & 0.617 & 0.600 & 0.573 & 0.571 & 0.567 & 0.400 \\
\hline 3.5 & 0.973 & 0.747 & 0.734 & 0.729 & 0.705 & 0.68 & 0.662 & 0.636 & 0.638 & 0.635 & 0.427 \\
\hline 4.5 & 0.99 & 0.806 & 0.791 & 0.788 & 0.765 & 0.737 & 0.719 & 0.694 & 0.698 & 0.697 & 0.477 \\
\hline 5.6 & 0.997 & 0.847 & 0.831 & 0.831 & 0.804 & 0.777 & 0.758 & 0.734 & 0.740 & 0.739 & 0.550 \\
\hline 7 & 0.999 & 0.868 & 0.863 & 0.862 & 0.837 & 0.810 & 0.789 & 0.765 & 0.774 & 0.774 & 0.580 \\
\hline 9 & 1.00 & 0.894 & 0.887 & 0.890 & 0.864 & 0.836 & 0.816 & 0.793 & 0.805 & 0.805 & 0.645 \\
\hline 11 & 1.00 & 0.910 & 0.901 & 0.904 & 0.881 & 0.852 & 0.832 & 0.811 & 0.822 & 0.824 & 0.727 \\
\hline 13 & 1.00 & 0.922 & 0.908 & 0.911 & 0.89 & 0.863 & 0.844 & 0.824 & 0.835 & 0.837 & 0.800 \\
\hline 15 & 1.00 & 0.931 & 0.913 & 0.915 & 0.895 & 0.869 & 0.851 & 0.833 & 0.845 & 0.847 & 0.860 \\
\hline 17.5 & 1.00 & 0.941 & 0.918 & 0.919 & 0.901 & 0.876 & 0.86 & 0.842 & 0.854 & 0.856 & 0.929 \\
\hline 20 & 1.00 & 0.949 & 0.923 & 0.923 & 0.906 & 0.883 & 0.867 & 0.850 & 0.861 & 0.863 & 1.00 \\
\hline 50 & 1.00 & 0.990 & 0.960 & 0.953 & 0.940 & 0.925 & 0.915 & 0.900 & 0.905 & 0.906 & 1.00 \\
\hline 100 & 1.00 & 0.999 & 0.986 & 0.978 & 0.967 & 0.955 & 0.948 & 0.936 & 0.937 & 0.936 & 1.00 \\
\hline 200 & 1.00 & 1.00 & 0.999 & 0.996 & 0.989 & 0.981 & 0.977 & 0.967 & 0.964 & 0.963 & 1.00 \\
\hline 500 & 1.00 & 1.00 & 1.00 & 1.00 & 1.00 & 0.999 & 0.998 & 0.993 & 0.990 & 0.988 & 1.00 \\
\hline 1000 & 1.00 & 1.00 & 1.00 & 1.00 & 1.00 & 1.00 & 1.00 & 1.00 & 0.999 & 0.998 & 1.00 \\
\hline
\end{tabular}




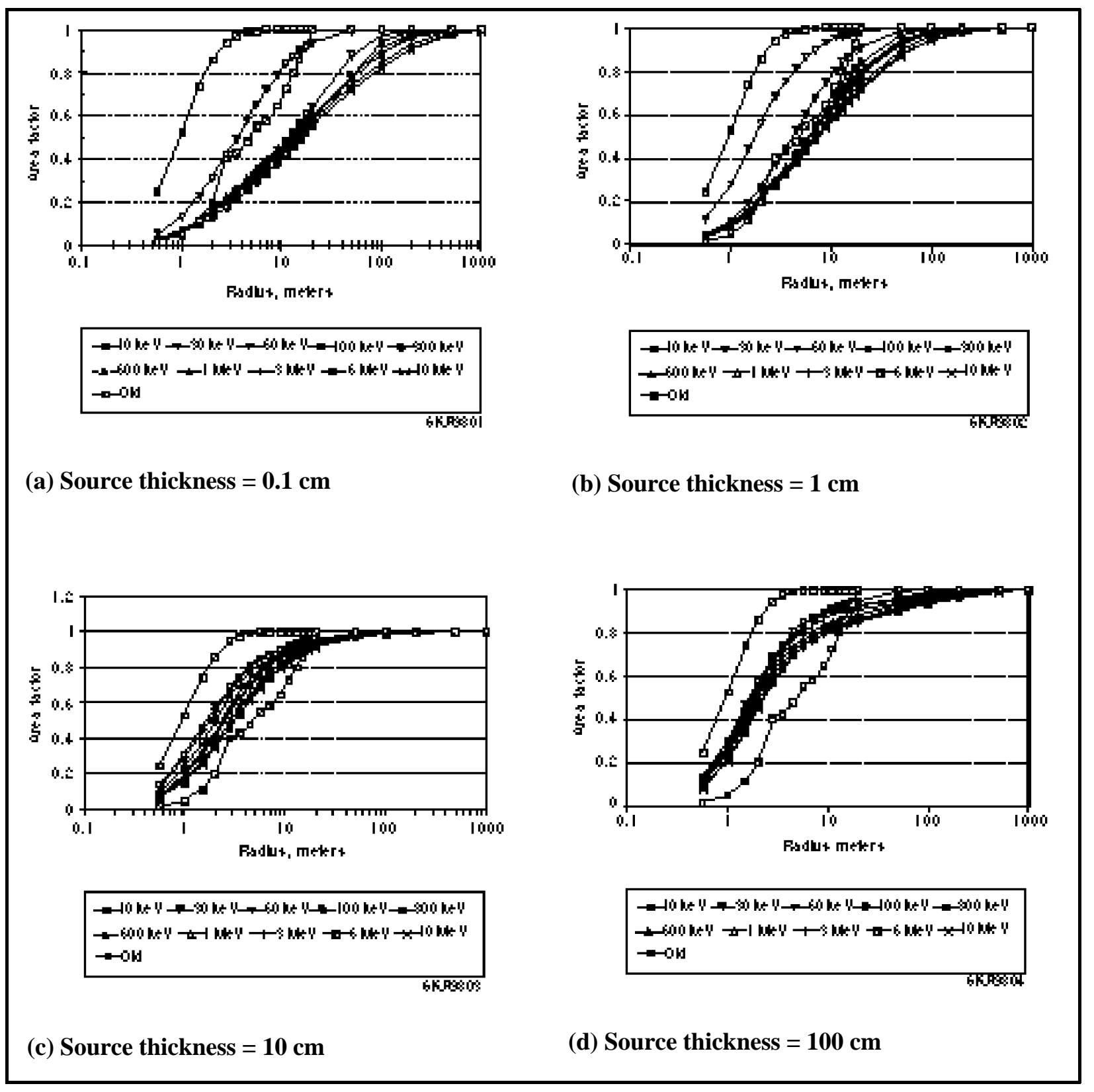

FIGURE 6 Area Factor Versus Source Radius for a Set of Gamma Energies with No Cover 
TABLE 12 Area Factor Variations with Cover Thickness for Different Source Radii at an Energy of $10 \mathrm{keV}$ and Source Depths of 0.1, 1, 10, and $100 \mathrm{~cm}$

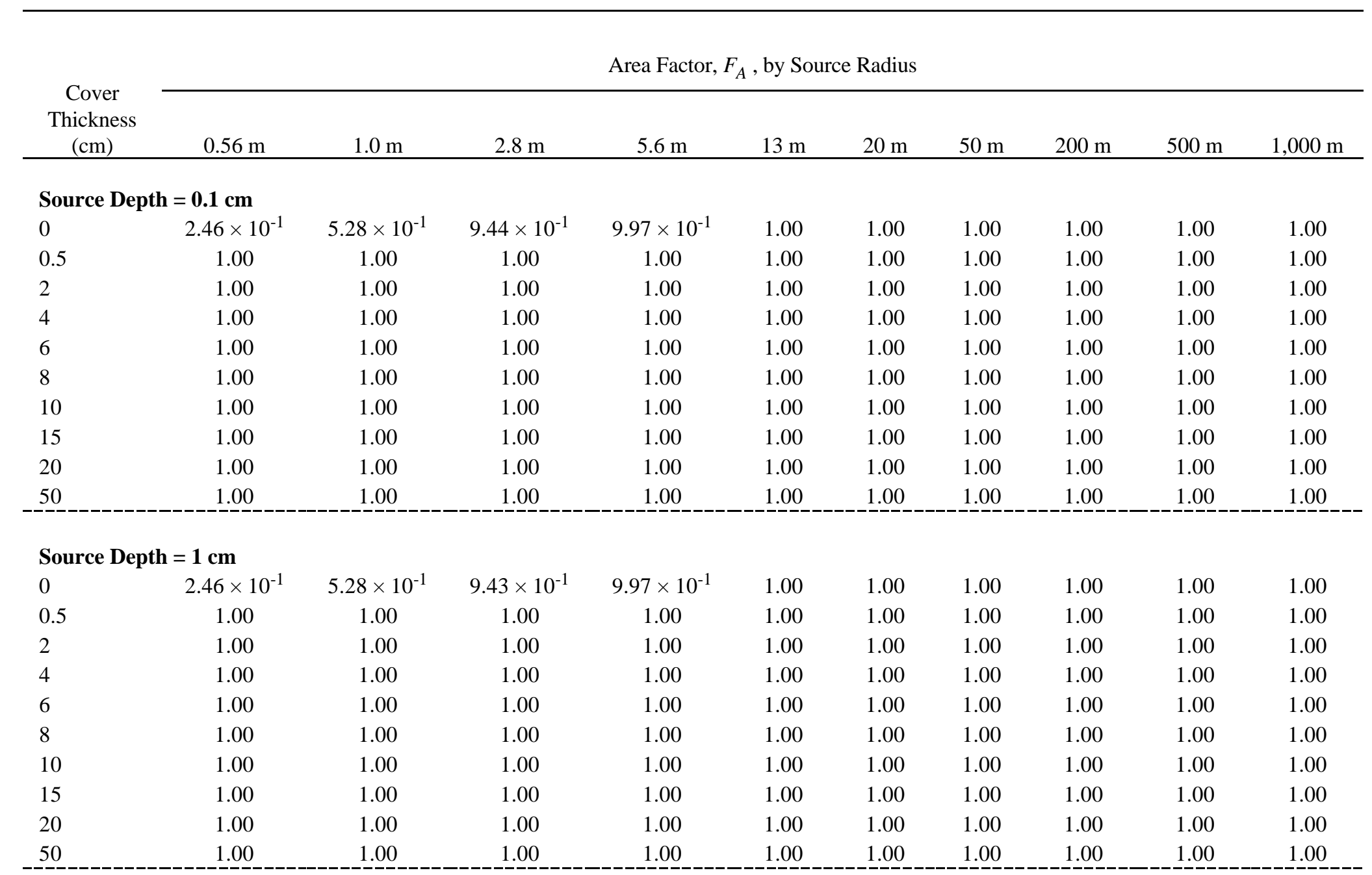


TABLE 12 (Cont.)

Area Factor, $F_{A}$, by Source Radius

\begin{tabular}{|c|c|c|c|c|c|c|c|c|c|c|}
\hline \multirow{2}{*}{$\begin{array}{c}\text { Cover } \\
\text { Thickness } \\
(\mathrm{cm})\end{array}$} & \\
\hline & $0.56 \mathrm{~m}$ & $1.0 \mathrm{~m}$ & $2.8 \mathrm{~m}$ & $5.6 \mathrm{~m}$ & $13 \mathrm{~m}$ & $20 \mathrm{~m}$ & $50 \mathrm{~m}$ & $200 \mathrm{~m}$ & $500 \mathrm{~m}$ & $1,000 \mathrm{~m}$ \\
\hline \multicolumn{11}{|c|}{ Source Depth $=10 \mathrm{~cm}$} \\
\hline 0 & $2.46 \times 10^{-1}$ & $5.28 \times 10^{-1}$ & $9.43 \times 10^{-1}$ & $9.97 \times 10^{-1}$ & 1.00 & 1.00 & 1.00 & 1.00 & 1.00 & 1.00 \\
\hline 0.5 & 1.00 & 1.00 & 1.00 & 1.00 & 1.00 & 1.00 & 1.00 & 1.00 & 1.00 & 1.00 \\
\hline 2 & 1.00 & 1.00 & 1.00 & 1.00 & 1.00 & 1.00 & 1.00 & 1.00 & 1.00 & 1.00 \\
\hline 4 & 1.00 & 1.00 & 1.00 & 1.00 & 1.00 & 1.00 & 1.00 & 1.00 & 1.00 & 1.00 \\
\hline 6 & 1.00 & 1.00 & 1.00 & 1.00 & 1.00 & 1.00 & 1.00 & 1.00 & 1.00 & 1.00 \\
\hline 8 & 1.00 & 1.00 & 1.00 & 1.00 & 1.00 & 1.00 & 1.00 & 1.00 & 1.00 & 1.00 \\
\hline 10 & 1.00 & 1.00 & 1.00 & 1.00 & 1.00 & 1.00 & 1.00 & 1.00 & 1.00 & 1.00 \\
\hline 15 & 1.00 & 1.00 & 1.00 & 1.00 & 1.00 & 1.00 & 1.00 & 1.00 & 1.00 & 1.00 \\
\hline 20 & 1.00 & 1.00 & 1.00 & 1.00 & 1.00 & 1.00 & 1.00 & 1.00 & 1.00 & 1.00 \\
\hline 50 & 1.00 & 1.00 & 1.00 & 1.00 & 1.00 & 1.00 & 1.00 & 1.00 & 1.00 & 1.00 \\
\hline \multicolumn{11}{|c|}{ Source Depth = $100 \mathrm{~cm}$} \\
\hline 0 & $2.46 \times 10^{-1}$ & $5.28 \times 10^{-1}$ & $9.43 \times 10^{-1}$ & $9.97 \times 10^{-1}$ & 1.00 & 1.00 & 1.00 & 1.00 & 1.00 & 1.00 \\
\hline 0.5 & 1.00 & 1.00 & 1.00 & 1.00 & 1.00 & 1.00 & 1.00 & 1.00 & 1.00 & 1.00 \\
\hline 2 & 1.00 & 1.00 & 1.00 & 1.00 & 1.00 & 1.00 & 1.00 & 1.00 & 1.00 & 1.00 \\
\hline 4 & 1.00 & 1.00 & 1.00 & 1.00 & 1.00 & 1.00 & 1.00 & 1.00 & 1.00 & 1.00 \\
\hline 6 & 1.00 & 1.00 & 1.00 & 1.00 & 1.00 & 1.00 & 1.00 & 1.00 & 1.00 & 1.00 \\
\hline 8 & 1.00 & 1.00 & 1.00 & 1.00 & 1.00 & 1.00 & 1.00 & 1.00 & 1.00 & 1.00 \\
\hline 10 & 1.00 & 1.00 & 1.00 & 1.00 & 1.00 & 1.00 & 1.00 & 1.00 & 1.00 & 1.00 \\
\hline 15 & 1.00 & 1.00 & 1.00 & 1.00 & 1.00 & 1.00 & 1.00 & 1.00 & 1.00 & 1.00 \\
\hline 20 & 1.00 & 1.00 & 1.00 & 1.00 & 1.00 & 1.00 & 1.00 & 1.00 & 1.00 & 1.00 \\
\hline 50 & 1.00 & 1.00 & 1.00 & 1.00 & 1.00 & 1.00 & 1.00 & 1.00 & 1.00 & 1.00 \\
\hline
\end{tabular}


TABLE 13 Area Factor Variations with Cover Thickness for Different Source Radii at an Energy of $100 \mathrm{keV}$ and Source Depths of $0.1,1,10$, and $100 \mathrm{~cm}$

\begin{tabular}{|c|c|c|c|c|c|c|c|c|c|c|}
\hline \multirow{2}{*}{$\begin{array}{c}\text { Cover } \\
\text { Thickness } \\
(\mathrm{cm})\end{array}$} & \multicolumn{10}{|c|}{ Area Factor, $F_{A}$, by Source Radius } \\
\hline & $0.56 \mathrm{~m}$ & $1.0 \mathrm{~m}$ & $2.8 \mathrm{~m}$ & $5.6 \mathrm{~m}$ & $13 \mathrm{~m}$ & $20 \mathrm{~m}$ & $50 \mathrm{~m}$ & $200 \mathrm{~m}$ & $500 \mathrm{~m}$ & $1,000 \mathrm{~m}$ \\
\hline \multicolumn{11}{|c|}{ Source Depth = $0.1 \mathrm{~cm}$} \\
\hline 0 & $2.18 \times 10^{-2}$ & $5.56 \times 10^{-2}$ & $1.78 \times 10^{-1}$ & $2.92 \times 10^{-1}$ & $4.57 \times 10^{-1}$ & $5.54 \times 10^{-1}$ & $7.89 \times 10^{-1}$ & $9.98 \times 10^{-1}$ & 1.00 & 1.00 \\
\hline 0.5 & $3.43 \times 10^{-2}$ & $8.88 \times 10^{-2}$ & $3.03 \times 10^{-1}$ & $5.24 \times 10^{-1}$ & $8.22 \times 10^{-1}$ & $9.39 \times 10^{-1}$ & 1.00 & 1.00 & 1.00 & 1.00 \\
\hline 2 & $6.69 \times 10^{-2}$ & $1.75 \times 10^{-1}$ & $5.86 \times 10^{-1}$ & $8.85 \times 10^{-1}$ & 1.00 & 1.00 & 1.00 & 1.00 & 1.00 & 1.00 \\
\hline 4 & $1.11 \times 10^{-1}$ & $2.84 \times 10^{-1}$ & $8.15 \times 10^{-1}$ & 1.00 & 1.00 & 1.00 & 1.00 & 1.00 & 1.00 & 1.00 \\
\hline 6 & $1.54 \times 10^{-1}$ & $3.81 \times 10^{-1}$ & $9.21 \times 10^{-1}$ & 1.00 & 1.00 & 1.00 & 1.00 & 1.00 & 1.00 & 1.00 \\
\hline 8 & $1.93 \times 10^{-1}$ & $4.64 \times 10^{-1}$ & $9.72 \times 10^{-1}$ & 1.00 & 1.00 & 1.00 & 1.00 & 1.00 & 1.00 & 1.00 \\
\hline 10 & $2.30 \times 10^{-1}$ & $5.34 \times 10^{-1}$ & $9.96 \times 10^{-1}$ & 1.00 & 1.00 & 1.00 & 1.00 & 1.00 & 1.00 & 1.00 \\
\hline 15 & $3.09 \times 10^{-1}$ & $6.65 \times 10^{-1}$ & 1.00 & 1.00 & 1.00 & 1.00 & 1.00 & 1.00 & 1.00 & 1.00 \\
\hline 20 & $3.73 \times 10^{-1}$ & $7.51 \times 10^{-1}$ & 1.00 & 1.00 & 1.00 & 1.00 & 1.00 & 1.00 & 1.00 & 1.00 \\
\hline 50 & $5.64 \times 10^{-1}$ & $9.24 \times 10^{-1}$ & 1.00 & 1.00 & 1.00 & 1.00 & 1.00 & 1.00 & 1.00 & 1.00 \\
\hline \multicolumn{11}{|c|}{ Source Depth $=1 \mathrm{~cm}$} \\
\hline 0 & $3.19 \times 10^{-2}$ & $8.26 \times 10^{-2}$ & $2.79 \times 10^{-1}$ & $4.76 \times 10^{-1}$ & $7.36 \times 10^{-1}$ & $8.43 \times 10^{-1}$ & $9.62 \times 10^{-1}$ & $9.99 \times 10^{-1}$ & 1.00 & 1.00 \\
\hline 0.5 & $4.33 \times 10^{-2}$ & $1.13 \times 10^{-1}$ & $3.91 \times 10^{-1}$ & $6.58 \times 10^{-1}$ & $9.29 \times 10^{-1}$ & $9.87 \times 10^{-1}$ & 1.00 & 1.00 & 1.00 & 1.00 \\
\hline 2 & $7.64 \times 10^{-2}$ & $1.99 \times 10^{-1}$ & $6.46 \times 10^{-1}$ & $9.26 \times 10^{-1}$ & 1.00 & 1.00 & 1.00 & 1.00 & 1.00 & 1.00 \\
\hline 4 & $1.20 \times 10^{-1}$ & $3.06 \times 10^{-1}$ & $8.43 \times 10^{-1}$ & 1.00 & 1.00 & 1.00 & 1.00 & 1.00 & 1.00 & 1.00 \\
\hline 6 & $1.62 \times 10^{-1}$ & $4.00 \times 10^{-1}$ & $9.35 \times 10^{-1}$ & 1.00 & 1.00 & 1.00 & 1.00 & 1.00 & 1.00 & 1.00 \\
\hline 8 & $2.02 \times 10^{-1}$ & $4.80 \times 10^{-1}$ & $9.79 \times 10^{-1}$ & 1.00 & 1.00 & 1.00 & 1.00 & 1.00 & 1.00 & 1.00 \\
\hline 10 & $2.37 \times 10^{-1}$ & $5.47 \times 10^{-1}$ & $9.98 \times 10^{-1}$ & 1.00 & 1.00 & 1.00 & 1.00 & 1.00 & 1.00 & 1.00 \\
\hline 15 & $3.15 \times 10^{-1}$ & $6.74 \times 10^{-1}$ & 1.00 & 1.00 & 1.00 & 1.00 & 1.00 & 1.00 & 1.00 & 1.00 \\
\hline 20 & $3.78 \times 10^{-1}$ & $7.57 \times 10^{-1}$ & 1.00 & 1.00 & 1.00 & 1.00 & 1.00 & 1.00 & 1.00 & 1.00 \\
\hline 50 & $5.65 \times 10^{-1}$ & $9.25 \times 10^{-1}$ & 1.00 & 1.00 & 1.00 & 1.00 & 1.00 & 1.00 & 1.00 & 1.00 \\
\hline
\end{tabular}


TABLE 13 (Cont.)

\begin{tabular}{|c|c|c|c|c|c|c|c|c|c|c|}
\hline \multirow{2}{*}{$\begin{array}{c}\text { Cover } \\
\text { Thickness } \\
(\mathrm{cm})\end{array}$} & \multicolumn{10}{|c|}{ Area Factor, $F_{A}$, by Source Radius } \\
\hline & $0.56 \mathrm{~m}$ & $1.0 \mathrm{~m}$ & $2.8 \mathrm{~m}$ & $5.6 \mathrm{~m}$ & $13 \mathrm{~m}$ & $20 \mathrm{~m}$ & $50 \mathrm{~m}$ & $200 \mathrm{~m}$ & $500 \mathrm{~m}$ & $1,000 \mathrm{~m}$ \\
\hline \multicolumn{11}{|c|}{ Source Depth $=10 \mathrm{~cm}$} \\
\hline 0 & $9.17 \times 10^{-2}$ & $2.31 \times 10^{-1}$ & $6.28 \times 10^{-1}$ & $8.16 \times 10^{-1}$ & $9.30 \times 10^{-1}$ & $9.58 \times 10^{-1}$ & $9.79 \times 10^{-1}$ & $9.98 \times 10^{-1}$ & 1.00 & 1.00 \\
\hline 0.5 & $1.05 \times 10^{-1}$ & $2.62 \times 10^{-1}$ & $7.02 \times 10^{-1}$ & $8.93 \times 10^{-1}$ & $9.84 \times 10^{-1}$ & $9.96 \times 10^{-1}$ & 1.00 & 1.00 & 1.00 & 1.00 \\
\hline 2 & $1.38 \times 10^{-1}$ & $3.39 \times 10^{-1}$ & $8.38 \times 10^{-1}$ & $9.83 \times 10^{-1}$ & 1.00 & 1.00 & 1.00 & 1.00 & 1.00 & 1.00 \\
\hline 4 & $1.78 \times 10^{-1}$ & $4.28 \times 10^{-1}$ & $9.32 \times 10^{-1}$ & 1.00 & 1.00 & 1.00 & 1.00 & 1.00 & 1.00 & 1.00 \\
\hline 6 & $2.16 \times 10^{-1}$ & $5.03 \times 10^{-1}$ & $9.75 \times 10^{-1}$ & 1.00 & 1.00 & 1.00 & 1.00 & 1.00 & 1.00 & 1.00 \\
\hline 8 & $2.50 \times 10^{-1}$ & $5.66 \times 10^{-1}$ & $9.94 \times 10^{-1}$ & 1.00 & 1.00 & 1.00 & 1.00 & 1.00 & 1.00 & 1.00 \\
\hline 10 & $2.82 \times 10^{-1}$ & $6.20 \times 10^{-1}$ & 1.00 & 1.00 & 1.00 & 1.00 & 1.00 & 1.00 & 1.00 & 1.00 \\
\hline 15 & $3.51 \times 10^{-1}$ & $7.21 \times 10^{-1}$ & 1.00 & 1.00 & 1.00 & 1.00 & 1.00 & 1.00 & 1.00 & 1.00 \\
\hline 20 & $4.06 \times 10^{-1}$ & $7.88 \times 10^{-1}$ & 1.00 & 1.00 & 1.00 & 1.00 & 1.00 & 1.00 & 1.00 & 1.00 \\
\hline$\underline{50}$ & $5.72 \times 10^{-1}$ & $9.29 \times 10^{-1}$ & 1.00 & 1.00 & 1.00 & 1.00 & 1.00 & 1.00 & 1.00 & 1.00 \\
\hline \multicolumn{11}{|c|}{ Source Depth = $100 \mathrm{~cm}$} \\
\hline 0 & $1.14 \times 10^{-1}$ & $2.74 \times 10^{-1}$ & $6.64 \times 10^{-1}$ & $8.31 \times 10^{-1}$ & $9.11 \times 10^{-1}$ & $9.23 \times 10^{-1}$ & $9.53 \times 10^{-1}$ & $9.96 \times 10^{-1}$ & 1.00 & 1.00 \\
\hline 0.5 & $1.28 \times 10^{-1}$ & $3.08 \times 10^{-1}$ & $7.37 \times 10^{-1}$ & $9.05 \times 10^{-1}$ & $9.77 \times 10^{-1}$ & $9.91 \times 10^{-1}$ & 1.00 & 1.00 & 1.00 & 1.00 \\
\hline 2 & $1.60 \times 10^{-1}$ & $3.80 \times 10^{-1}$ & $8.57 \times 10^{-1}$ & $9.84 \times 10^{-1}$ & 1.00 & 1.00 & 1.00 & 1.00 & 1.00 & 1.00 \\
\hline 4 & $1.98 \times 10^{-1}$ & $4.61 \times 10^{-1}$ & $9.41 \times 10^{-1}$ & 1.00 & 1.00 & 1.00 & 1.00 & 1.00 & 1.00 & 1.00 \\
\hline 6 & $2.34 \times 10^{-1}$ & $5.31 \times 10^{-1}$ & $9.78 \times 10^{-1}$ & 1.00 & 1.00 & 1.00 & 1.00 & 1.00 & 1.00 & 1.00 \\
\hline 8 & $2.66 \times 10^{-1}$ & $5.89 \times 10^{-1}$ & $9.94 \times 10^{-1}$ & 1.00 & 1.00 & 1.00 & 1.00 & 1.00 & 1.00 & 1.00 \\
\hline 10 & $2.97 \times 10^{-1}$ & $6.39 \times 10^{-1}$ & 1.00 & 1.00 & 1.00 & 1.00 & 1.00 & 1.00 & 1.00 & 1.00 \\
\hline 15 & $3.62 \times 10^{-1}$ & $7.34 \times 10^{-1}$ & 1.00 & 1.00 & 1.00 & 1.00 & 1.00 & 1.00 & 1.00 & 1.00 \\
\hline 20 & $4.14 \times 10^{-1}$ & $7.97 \times 10^{-1}$ & 1.00 & 1.00 & 1.00 & 1.00 & 1.00 & 1.00 & 1.00 & 1.00 \\
\hline 50 & $5.74 \times 10^{-1}$ & $9.30 \times 10^{-1}$ & 1.00 & 1.00 & 1.00 & 1.00 & 1.00 & 1.00 & 1.00 & 1.00 \\
\hline
\end{tabular}


TABLE 14 Area Factor Variations with Cover Thickness for Different Source Radii at an Energy of 1 MeV and Source Depths of $0.1,1,10$, and $100 \mathrm{~cm}$

\begin{tabular}{|c|c|c|c|c|c|c|c|c|c|c|}
\hline \multirow{2}{*}{$\begin{array}{c}\text { Cover } \\
\text { Thickness } \\
(\mathrm{cm})\end{array}$} & \multicolumn{10}{|c|}{ Area Factor, $F_{A}$, by Source Radius } \\
\hline & $0.56 \mathrm{~m}$ & $1.0 \mathrm{~m}$ & $2.8 \mathrm{~m}$ & $5.6 \mathrm{~m}$ & $13 \mathrm{~m}$ & $20 \mathrm{~m}$ & $50 \mathrm{~m}$ & $200 \mathrm{~m}$ & $500 \mathrm{~m}$ & $1,000 \mathrm{~m}$ \\
\hline \multicolumn{11}{|c|}{ Source Depth $=0.1 \mathrm{~cm}$} \\
\hline 0 & $2.78 \times 10^{-2}$ & $7.05 \times 10^{-2}$ & $2.22 \times 10^{-1}$ & $3.53 \times 10^{-1}$ & $5.19 \times 10^{-1}$ & $6.04 \times 10^{-1}$ & $7.76 \times 10^{-1}$ & $9.66 \times 10^{-1}$ & $9.99 \times 10^{-1}$ & 1.00 \\
\hline 0.5 & $4.05 \times 10^{-2}$ & $1.03 \times 10^{-1}$ & $3.23 \times 10^{-1}$ & $5.11 \times 10^{-1}$ & $7.36 \times 10^{-1}$ & $8.36 \times 10^{-1}$ & $9.71 \times 10^{-1}$ & 1.00 & 1.00 & 1.00 \\
\hline 2 & $5.98 \times 10^{-2}$ & $1.52 \times 10^{-1}$ & $4.71 \times 10^{-1}$ & $7.18 \times 10^{-1}$ & $9.26 \times 10^{-1}$ & $9.85 \times 10^{-1}$ & 1.00 & 1.00 & 1.00 & 1.00 \\
\hline 4 & $8.11 \times 10^{-2}$ & $2.06 \times 10^{-1}$ & $6.17 \times 10^{-1}$ & $8.77 \times 10^{-1}$ & $9.99 \times 10^{-1}$ & 1.00 & 1.00 & 1.00 & 1.00 & 1.00 \\
\hline 6 & $9.84 \times 10^{-2}$ & $2.49 \times 10^{-1}$ & $7.14 \times 10^{-1}$ & $9.61 \times 10^{-1}$ & 1.00 & 1.00 & 1.00 & 1.00 & 1.00 & 1.00 \\
\hline 8 & $1.14 \times 10^{-1}$ & $2.87 \times 10^{-1}$ & $7.88 \times 10^{-1}$ & $9.76 \times 10^{-1}$ & 1.00 & 1.00 & 1.00 & 1.00 & 1.00 & 1.00 \\
\hline 10 & $1.29 \times 10^{-1}$ & $3.22 \times 10^{-1}$ & $8.38 \times 10^{-1}$ & $9.92 \times 10^{-1}$ & 1.00 & 1.00 & 1.00 & 1.00 & 1.00 & 1.00 \\
\hline 15 & $1.61 \times 10^{-1}$ & $3.94 \times 10^{-1}$ & $9.18 \times 10^{-1}$ & 1.00 & 1.00 & 1.00 & 1.00 & 1.00 & 1.00 & 1.00 \\
\hline 20 & $1.87 \times 10^{-1}$ & $4.52 \times 10^{-1}$ & $9.57 \times 10^{-1}$ & 1.00 & 1.00 & 1.00 & 1.00 & 1.00 & 1.00 & 1.00 \\
\hline 50 & $2.77 \times 10^{-1}$ & $6.24 \times 10^{-1}$ & 1.00 & 1.00 & 1.00 & 1.00 & 1.00 & 1.00 & 1.00 & 1.00 \\
\hline \multicolumn{11}{|c|}{ Source Depth $=1 \mathrm{~cm}$} \\
\hline 0 & $3.78 \times 10^{-2}$ & $9.61 \times 10^{-2}$ & $3.02 \times 10^{-1}$ & $4.77 \times 10^{-1}$ & $6.88 \times 10^{-1}$ & $7.83 \times 10^{-1}$ & $9.28 \times 10^{-1}$ & $9.95 \times 10^{-1}$ & $9.98 \times 10^{-1}$ & 1.00 \\
\hline 0.5 & $4.73 \times 10^{-2}$ & $1.20 \times 10^{-1}$ & $3.76 \times 10^{-1}$ & $5.89 \times 10^{-1}$ & $8.23 \times 10^{-1}$ & $9.11 \times 10^{-1}$ & $9.94 \times 10^{-1}$ & 1.00 & 1.00 & 1.00 \\
\hline 2 & $6.56 \times 10^{-2}$ & $1.67 \times 10^{-1}$ & $5.14 \times 10^{-1}$ & $7.72 \times 10^{-1}$ & $9.68 \times 10^{-1}$ & 1.00 & 1.00 & 1.00 & 1.00 & 1.00 \\
\hline 4 & $8.45 \times 10^{-2}$ & $2.15 \times 10^{-1}$ & $6.37 \times 10^{-1}$ & $8.91 \times 10^{-1}$ & 1.00 & 1.00 & 1.00 & 1.00 & 1.00 & 1.00 \\
\hline 6 & $1.02 \times 10^{-1}$ & $2.58 \times 10^{-1}$ & $7.32 \times 10^{-1}$ & $9.58 \times 10^{-1}$ & 1.00 & 1.00 & 1.00 & 1.00 & 1.00 & 1.00 \\
\hline 8 & $1.17 \times 10^{-1}$ & $2.95 \times 10^{-1}$ & $7.99 \times 10^{-1}$ & $9.81 \times 10^{-1}$ & 1.00 & 1.00 & 1.00 & 1.00 & 1.00 & 1.00 \\
\hline 10 & $1.32 \times 10^{-1}$ & $3.29 \times 10^{-1}$ & $8.47 \times 10^{-1}$ & $9.94 \times 10^{-1}$ & 1.00 & 1.00 & 1.00 & 1.00 & 1.00 & 1.00 \\
\hline 15 & $1.63 \times 10^{-1}$ & $4.00 \times 10^{-1}$ & $9.23 \times 10^{-1}$ & 1.00 & 1.00 & 1.00 & 1.00 & 1.00 & 1.00 & 1.00 \\
\hline 20 & $1.89 \times 10^{-1}$ & $4.56 \times 10^{-1}$ & $9.60 \times 10^{-1}$ & 1.00 & 1.00 & 1.00 & 1.00 & 1.00 & 1.00 & 1.00 \\
\hline 50 & $2.78 \times 10^{-1}$ & $6.25 \times 10^{-1}$ & 1.00 & 1.00 & 1.00 & 1.00 & 1.00 & 1.00 & 1.00 & 1.00 \\
\hline
\end{tabular}


TABLE 14 (Cont.)

\begin{tabular}{|c|c|c|c|c|c|c|c|c|c|c|}
\hline \multirow{2}{*}{$\begin{array}{c}\text { Cover } \\
\text { Thickness } \\
(\mathrm{cm})\end{array}$} & \multicolumn{10}{|c|}{ Area Factor, $F_{A}$, by Source Radius } \\
\hline & $0.56 \mathrm{~m}$ & $1.0 \mathrm{~m}$ & $2.8 \mathrm{~m}$ & $5.6 \mathrm{~m}$ & $13 \mathrm{~m}$ & $20 \mathrm{~m}$ & $50 \mathrm{~m}$ & $200 \mathrm{~m}$ & $500 \mathrm{~m}$ & $1,000 \mathrm{~m}$ \\
\hline \multicolumn{11}{|c|}{ Source Depth $=10 \mathrm{~cm}$} \\
\hline 0 & $7.20 \times 10^{-2}$ & $1.82 \times 10^{-1}$ & $5.37 \times 10^{-1}$ & $7.54 \times 10^{-1}$ & $8.99 \times 10^{-1}$ & $9.35 \times 10^{-1}$ & $9.76 \times 10^{-1}$ & $9.95 \times 10^{-1}$ & $9.99 \times 10^{-1}$ & 1.00 \\
\hline 0.5 & $8.02 \times 10^{-2}$ & $2.03 \times 10^{-1}$ & $5.92 \times 10^{-1}$ & $8.21 \times 10^{-1}$ & $9.54 \times 10^{-1}$ & $9.81 \times 10^{-1}$ & $9.99 \times 10^{-1}$ & 1.00 & 1.00 & 1.00 \\
\hline 2 & $9.51 \times 10^{-2}$ & $2.40 \times 10^{-1}$ & $6.79 \times 10^{-1}$ & $9.00 \times 10^{-1}$ & $9.93 \times 10^{-1}$ & $9.99 \times 10^{-1}$ & 1.00 & 1.00 & 1.00 & 1.00 \\
\hline 4 & $1.13 \times 10^{-1}$ & $2.82 \times 10^{-1}$ & $7.65 \times 10^{-1}$ & $9.59 \times 10^{-1}$ & 1.00 & 1.00 & 1.00 & 1.00 & 1.00 & 1.00 \\
\hline 6 & $1.28 \times 10^{-1}$ & $3.19 \times 10^{-1}$ & $8.26 \times 10^{-1}$ & $9.84 \times 10^{-1}$ & 1.00 & 1.00 & 1.00 & 1.00 & 1.00 & 1.00 \\
\hline 8 & $1.42 \times 10^{-1}$ & $3.51 \times 10^{-1}$ & $8.69 \times 10^{-1}$ & $9.95 \times 10^{-1}$ & 1.00 & 1.00 & 1.00 & 1.00 & 1.00 & 1.00 \\
\hline 10 & $1.54 \times 10^{-1}$ & $3.80 \times 10^{-1}$ & $9.01 \times 10^{-1}$ & $9.99 \times 10^{-1}$ & 1.00 & 1.00 & 1.00 & 1.00 & 1.00 & 1.00 \\
\hline 15 & $1.82 \times 10^{-1}$ & $4.41 \times 10^{-1}$ & $9.49 \times 10^{-1}$ & 1.00 & 1.00 & 1.00 & 1.00 & 1.00 & 1.00 & 1.00 \\
\hline 20 & $2.05 \times 10^{-1}$ & $4.89 \times 10^{-1}$ & $9.74 \times 10^{-1}$ & 1.00 & 1.00 & 1.00 & 1.00 & 1.00 & 1.00 & 1.00 \\
\hline$\underline{50}$ & $2.84 \times 10^{-1}$ & $6.34 \times 10^{-1}$ & 1.00 & 1.00 & 1.00 & 1.00 & 1.00 & 1.00 & 1.00 & 1.00 \\
\hline \multicolumn{11}{|c|}{ Source Depth = $100 \mathrm{~cm}$} \\
\hline 0 & $9.55 \times 10^{-2}$ & $2.36 \times 10^{-1}$ & $6.00 \times 10^{-1}$ & $7.58 \times 10^{-1}$ & $8.44 \times 10^{-1}$ & $8.67 \times 10^{-1}$ & $9.15 \times 10^{-1}$ & $9.77 \times 10^{-1}$ & $9.98 \times 10^{-1}$ & 1.00 \\
\hline 0.5 & $1.10 \times 10^{-1}$ & $2.71 \times 10^{-1}$ & $6.84 \times 10^{-1}$ & $8.56 \times 10^{-1}$ & $9.41 \times 10^{-1}$ & $9.63 \times 10^{-1}$ & $9.94 \times 10^{-1}$ & 1.00 & 1.00 & 1.00 \\
\hline 2 & $1.26 \times 10^{-1}$ & $3.11 \times 10^{-1}$ & $7.66 \times 10^{-1}$ & $9.29 \times 10^{-1}$ & $9.86 \times 10^{-1}$ & $9.97 \times 10^{-1}$ & 1.00 & 1.00 & 1.00 & 1.00 \\
\hline 4 & $1.43 \times 10^{-1}$ & $3.50 \times 10^{-1}$ & $8.34 \times 10^{-1}$ & $9.72 \times 10^{-1}$ & 1.00 & 1.00 & 1.00 & 1.00 & 1.00 & 1.00 \\
\hline 6 & $1.57 \times 10^{-1}$ & $3.82 \times 10^{-1}$ & $8.77 \times 10^{-1}$ & $9.92 \times 10^{-1}$ & 1.00 & 1.00 & 1.00 & 1.00 & 1.00 & 1.00 \\
\hline 8 & $1.69 \times 10^{-1}$ & $4.09 \times 10^{-1}$ & $9.09 \times 10^{-1}$ & $9.97 \times 10^{-1}$ & 1.00 & 1.00 & 1.00 & 1.00 & 1.00 & 1.00 \\
\hline 10 & $1.89 \times 10^{-1}$ & $4.34 \times 10^{-1}$ & $9.31 \times 10^{-1}$ & $9.99 \times 10^{-1}$ & 1.00 & 1.00 & 1.00 & 1.00 & 1.00 & 1.00 \\
\hline 15 & $2.04 \times 10^{-1}$ & $4.84 \times 10^{-1}$ & $9.65 \times 10^{-1}$ & 1.00 & 1.00 & 1.00 & 1.00 & 1.00 & 1.00 & 1.00 \\
\hline 20 & $2.24 \times 10^{-1}$ & $5.25 \times 10^{-1}$ & $9.83 \times 10^{-1}$ & 1.00 & 1.00 & 1.00 & 1.00 & 1.00 & 1.00 & 1.00 \\
\hline 50 & $2.90 \times 10^{-1}$ & $6.45 \times 10^{-1}$ & 1.00 & 1.00 & 1.00 & 1.00 & 1.00 & 1.00 & 1.00 & 1.00 \\
\hline
\end{tabular}


Figure 7a shows the area factor variation with cover thickness for a $10-\mathrm{keV}$ source at depths of 0.1 , $1.0,10.0$, and $100.0 \mathrm{~cm}$. As shown, for a $10-\mathrm{keV}$ source, the area factor is independent of source depth.

Table 13 gives the area factor variation at $100 \mathrm{keV}$. The cover thickness varies from 0 to $50 \mathrm{~cm}$, and the area factor is calculated at different source radii $(0.56,1.0,2.8,5.6,13.0,20.0,50.0$, 200.0, 500.0, and $1000 \mathrm{~m}$ ). Results are shown in Figures 7b through 7e. Table 13 shows that for source radii greater than $200 \mathrm{~m}$, the area factor is always 1 . As the cover thickness increases, the area factor approaches unity at smaller source radii. Table 14 shows the area factor variation at $1 \mathrm{MeV}$.

Tables 12 to 14 and Figure 7 show that for very low energy $(10 \mathrm{keV})$, the area factor is a function of source radii; however, with a very small cover, the area factor becomes independent of source radii and becomes unity. For energies in the range of 100 to $1,000 \mathrm{keV}$, the area factor

increases with source radius and cover thickness. As the cover thickness increases, the area factor saturates at a smaller source radius.

\subsection{DOSE CALCULATIONS}

Doses were calculated for four source depths $(1,5,15$, and $50 \mathrm{~cm})$ with the old and new RESRAD models for selected radionuclides without any cover. Table 15 compares results of the new dose model with those of the old model for Cs-137, Co-60, Mn-54, Co-57, U-234, U-235, U-238, and Al-26. In these calculations, the source radius varies from 0.56 to $1,000 \mathrm{~m}$. Doses $[(\mathrm{mrem} / \mathrm{yr}) /(\mathrm{pCi} / \mathrm{g})]$ are compared only for the external pathway at time zero. The following RESRAD parameters were used in the calculations:

C Density of contaminated zone $=1.6 \mathrm{~g} / \mathrm{cm}^{3}$,

C Cover density $=1.6 \mathrm{~g} / \mathrm{cm}^{3}$,

C Initial concentration of radionuclides $=1 \mathrm{pCi} / \mathrm{g}$,

C Exposure duration $=30$ years,

C External shielding factor $=1$,

C Time fraction for outdoors $=1$, and

C Time fraction for indoors $=0$. 


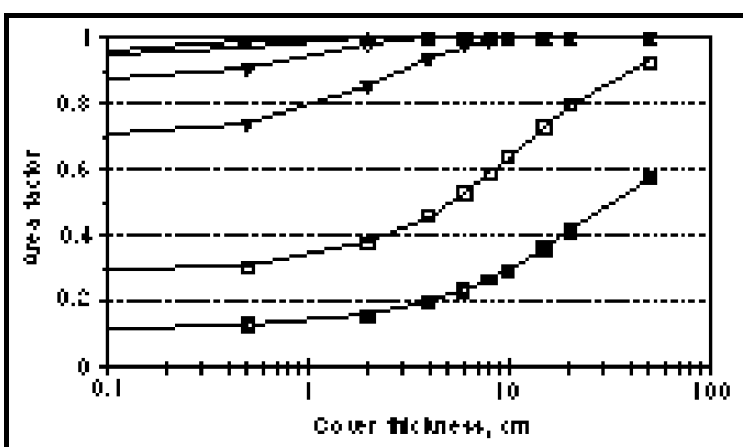

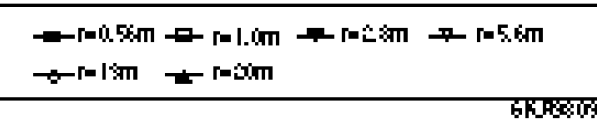

(e) Source Depth of $100.0 \mathrm{~cm}$ and Energy of $100 \mathrm{keV}$

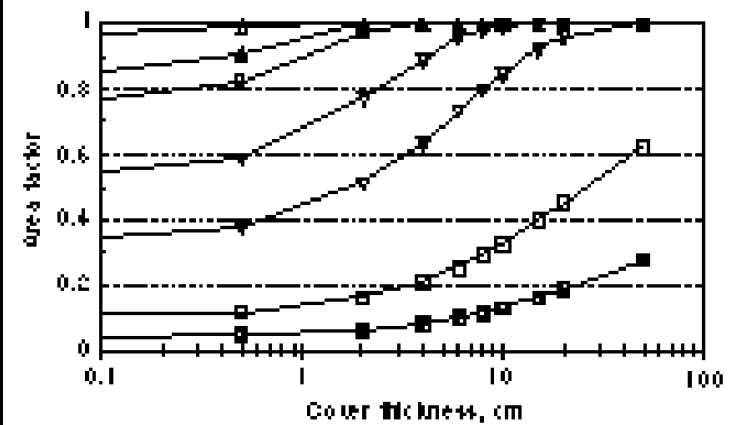

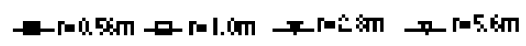

$\rightarrow-r$ İm t

GFHET

(g) Source Depth of $1.0 \mathrm{~cm}$ and Energy of $1 \mathrm{MeV}$

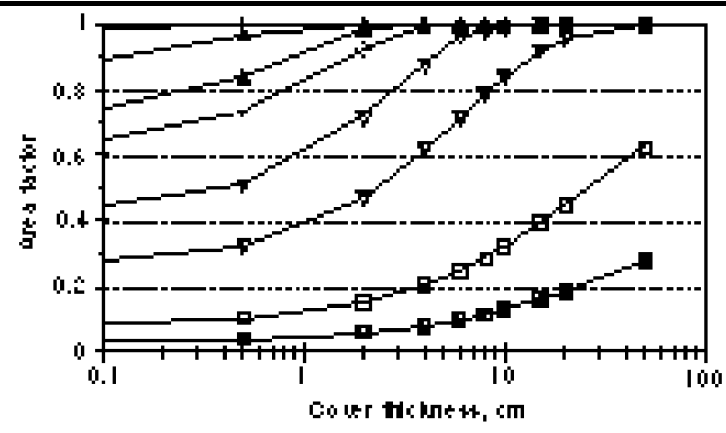

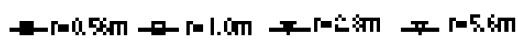

- เ-r-I:m -

GFHOT

(f) Source Depth of $0.1 \mathrm{~cm}$ and Energy of $1 \mathrm{MeV}$

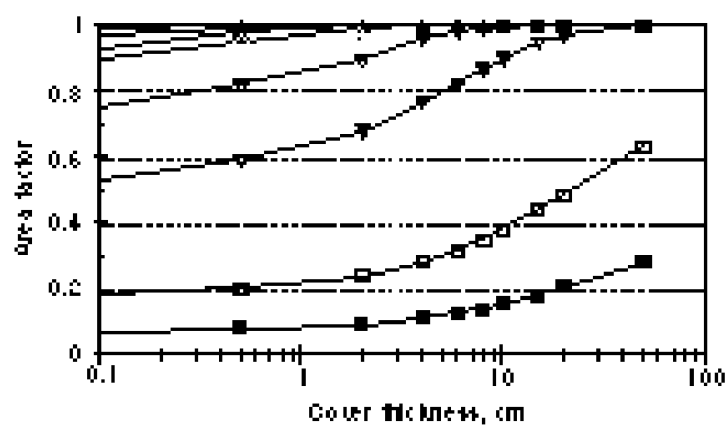

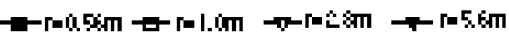

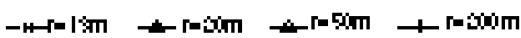
BRAESTE

(h) Source Depth of $10.0 \mathrm{~cm}$ and Energy of $1 \mathrm{MeV}$

\section{FIGURE 7 (Cont.)}




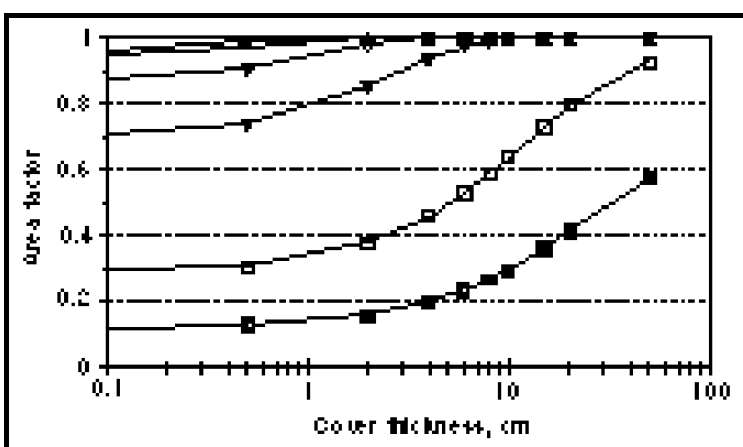

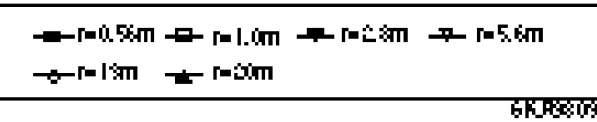

(e) Source Depth of $100.0 \mathrm{~cm}$ and Energy of $100 \mathrm{keV}$

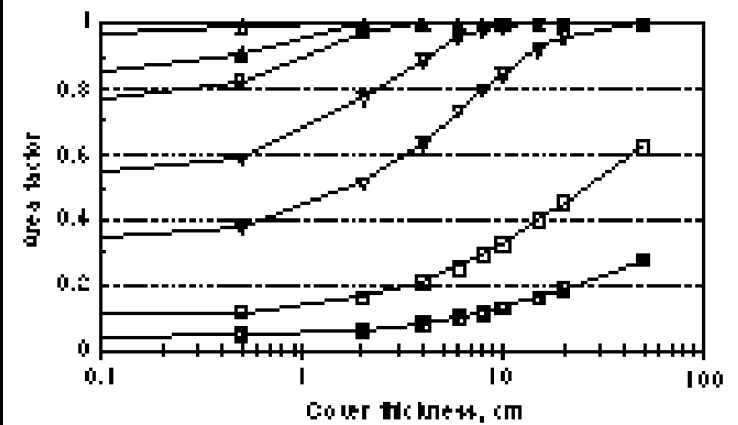

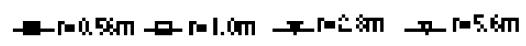

$\rightarrow-r$ İm t

GFHET

(g) Source Depth of $1.0 \mathrm{~cm}$ and Energy of $1 \mathrm{MeV}$

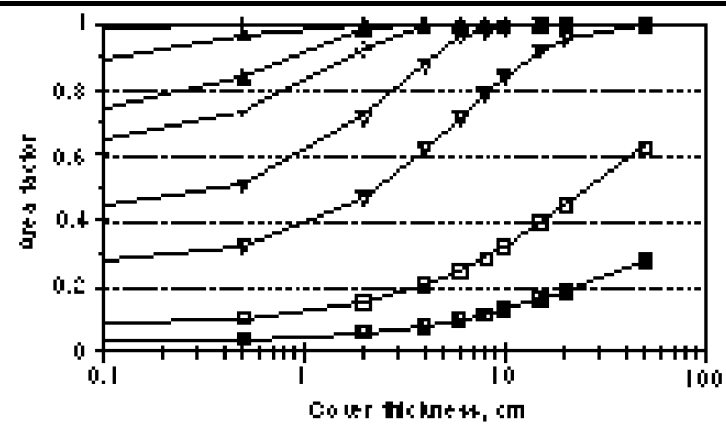

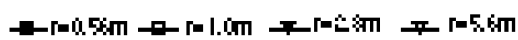

- เ-r-I:m -

GFHOT

(f) Source Depth of $0.1 \mathrm{~cm}$ and Energy of $1 \mathrm{MeV}$

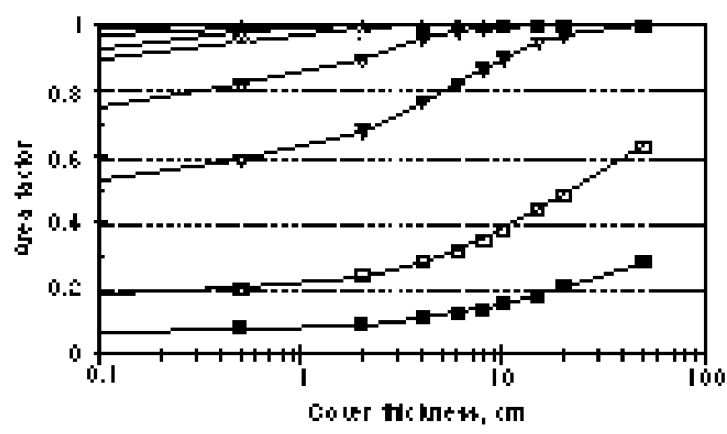

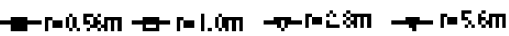

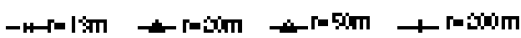
BRAESTE

(h) Source Depth of $10.0 \mathrm{~cm}$ and Energy of $1 \mathrm{MeV}$

\section{FIGURE 7 (Cont.)}




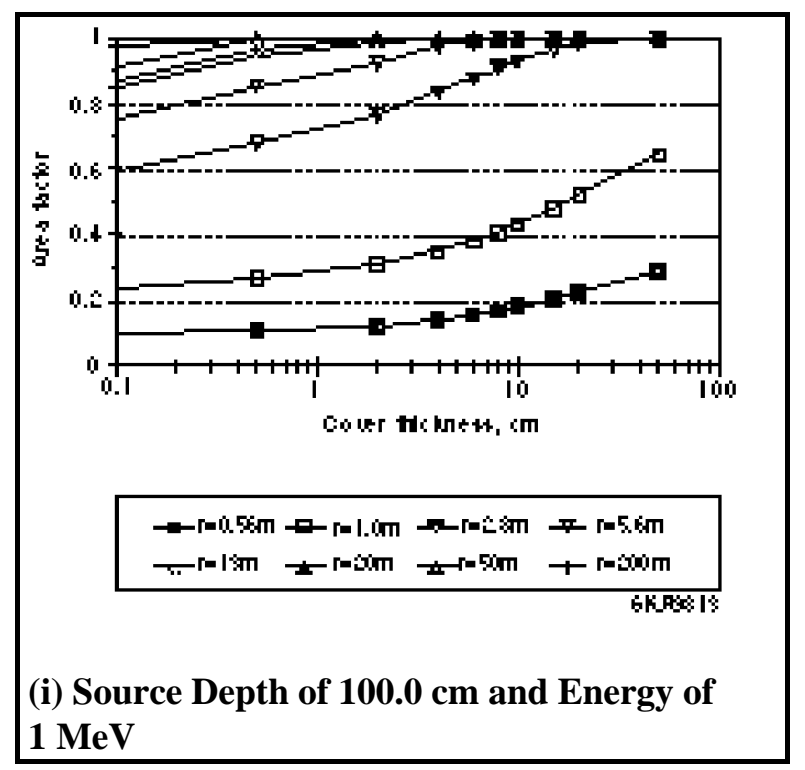

\section{FIGURE 7 (Cont.)}

Contaminated zone parameters, such as erosion rate, total porosity, effective porosity, hydraulic conductivity, $b$ parameter, evapotranspiration coefficient, precipitation, irrigation mode, and runoff coefficients, will not affect these calculations.

Figures 8a through $8 \mathrm{~h}$ compare doses between the old and new RESRAD models for Cs-137, Co-60, Co-57, Mn-54, U-234, U-235, U-238, and Al-26. These figures show the combined effects of depth factor, area factor, and $D C F$ differences in the two models on dose calculations. For a small radius and 50-cm-thick source, differences are due to the area factor and $D C F$; however, at a large radius, the difference arises only because of the $D C F$.

Figure 8a compares Cs-137 doses in the two models. With the new model, the DCF is $3.42 \mathrm{mrem} / \mathrm{yr}$; with the old model, it is 5.03 . This change of about $47 \%$ is reflected in the dose value for a source depth of $50 \mathrm{~cm}$ and a 1,000-m radius. Based solely on the $D C F$ difference, all the values at a source depth of $50 \mathrm{~cm}$ in the old RESRAD model should have been larger; however, it is not so because of differences in area factor. The new model always gives a large area factor at a small radius compared with the old model; this fact is reflected in dose values at a small radius. In Figures 8a through $8 \mathrm{~h}$, dose values at $0.56-\mathrm{m}$ radius are always higher for the new model. The ratio in the new model at $0.56-\mathrm{m}$ radius increases as the source thickness increases. The old model dose values do not change beyond a 20-m radius; for the new model, saturation occurs beyond a $20-\mathrm{m}$ radius and depends on source energy. For Co-57, dose values change as much as 5 to $24 \%$, depending on the source thickness, when the radius is increased beyond $20 \mathrm{~m}$. For Al-26, this change is 10 to $32 \%$. 
TABLE 15 Dose (mrem/yr) Comparisons for Radionuclides at Source Depths $\left(T_{s}\right)$ of $1,5,15$, and $50 \mathrm{~cm}$ for New and Old RESRAD Models

\begin{tabular}{|c|c|c|c|c|c|c|c|c|}
\hline \multirow{2}{*}{$\begin{array}{c}\text { Radius } \\
(\mathrm{m})\end{array}$} & \multicolumn{4}{|c|}{ New Model } & \multicolumn{4}{|c|}{ Old Model } \\
\hline & $T_{s}=1 \mathrm{~cm}$ & $T s=5 \mathrm{~cm}$ & $T s=15 \mathrm{~cm}$ & $T s=50 \mathrm{~cm}$ & $T s=1 \mathrm{~cm}$ & $T s=5 \mathrm{~cm}$ & $T s=15 \mathrm{~cm}$ & $T s=50 \mathrm{~cm}$ \\
\hline \multicolumn{9}{|l|}{ Cs-137 } \\
\hline 0.56 & $2.54 \times 10^{-2}$ & $1.16 \times 10^{-1}$ & $2.69 \times 10^{-1}$ & $3.48 \times 10^{-1}$ & $1.01 \times 10^{-2}$ & $3.92 \times 10^{-2}$ & $7.00 \times 10^{-2}$ & $8.10 \times 10^{-2}$ \\
\hline 2.8 & $1.96 \times 10^{-1}$ & $8.78 \times 10^{-1}$ & 1.82 & 2.09 & $2.51 \times 10^{-1}$ & $9.81 \times 10^{-1}$ & 1.75 & 2.03 \\
\hline 5.6 & $3.15 \times 10^{-1}$ & 1.33 & 2.45 & 2.67 & $3.49 \times 10^{-1}$ & 1.36 & 2.43 & 2.81 \\
\hline 13 & $4.55 \times 10^{-1}$ & 1.69 & 2.82 & 2.97 & $5.16 \times 10^{-1}$ & 2.01 & 3.59 & 4.16 \\
\hline 20 & $5.11 \times 10^{-1}$ & 1.78 & 2.91 & 3.04 & $6.37 \times 10^{-1}$ & 2.49 & 4.44 & 5.14 \\
\hline 564.19 & $6.66 \times 10^{-1}$ & 1.93 & 3.09 & 3.41 & $6.37 \times 10^{-1}$ & 2.49 & 4.44 & 5.14 \\
\hline \multicolumn{9}{|l|}{ Co-60 } \\
\hline 0.56 & $1.08 \times 10^{-1}$ & $4.76 \times 10^{-1}$ & 1.16 & 1.60 & $3.89 \times 10^{-2}$ & $1.57 \times 10^{-1}$ & $2.98 \times 10^{-1}$ & $3.65 \times 10^{-1}$ \\
\hline 2.8 & $8.33 \times 10^{-1}$ & 3.64 & 8.02 & 9.88 & $9.74 \times 10^{-1}$ & 3.94 & 7.46 & 9.12 \\
\hline 5.6 & 1.33 & 5.55 & $1.10 \times 10^{1}$ & $1.27 \times 10^{1}$ & 1.35 & 5.46 & $1.04 \times 10^{1}$ & $1.27 \times 10^{1}$ \\
\hline 13 & 1.91 & 7.10 & $1.27 \times 10^{1}$ & $1.42 \times 10^{1}$ & 2.00 & 8.08 & $1.53 \times 10^{1}$ & $1.87 \times 10^{1}$ \\
\hline 20 & 2.15 & 7.49 & $1.31 \times 10^{1}$ & $1.45 \times 10^{1}$ & 2.47 & 9.99 & $1.89 \times 10^{1}$ & $2.31 \times 10^{1}$ \\
\hline 564.19 & 2.83 & 8.20 & $1.39 \times 10^{1}$ & $1.62 \times 10^{1}$ & 2.47 & 9.99 & $1.89 \times 10^{1}$ & $2.31 \times 10^{1}$ \\
\hline \multicolumn{9}{|l|}{ Co-57 } \\
\hline 0.56 & $4.44 \times 10^{-3}$ & $2.45 \times 10^{-2}$ & $5.24 \times 10^{-2}$ & $5.75 \times 10^{-2}$ & $1.64 \times 10^{-3}$ & $5.51 \times 10^{-3}$ & $7.89 \times 10^{-3}$ & $8.18 \times 10^{-3}$ \\
\hline 2.8 & $3.78 \times 10^{-2}$ & $1.94 \times 10^{-1}$ & $3.19 \times 10^{-1}$ & $3.28 \times 10^{-1}$ & $4.11 \times 10^{-2}$ & $1.38 \times 10^{-1}$ & $1.97 \times 10^{-1}$ & $2.04 \times 10^{-1}$ \\
\hline 5.6 & $6.43 \times 10^{-2}$ & $2.83 \times 10^{-1}$ & $4.08 \times 10^{-1}$ & $4.16 \times 10^{-1}$ & $5.70 \times 10^{-2}$ & $1.91 \times 10^{-1}$ & $2.74 \times 10^{-1}$ & $2.84 \times 10^{-1}$ \\
\hline 13 & $9.77 \times 10^{-2}$ & $3.38 \times 10^{-1}$ & $4.57 \times 10^{-1}$ & $4.57 \times 10^{-1}$ & $8.43 \times 10^{-2}$ & $2.83 \times 10^{-1}$ & $4.05 \times 10^{-1}$ & $4.20 \times 10^{-1}$ \\
\hline 20 & $1.11 \times 10^{-1}$ & $3.51 \times 10^{-1}$ & $4.67 \times 10^{-1}$ & $4.62 \times 10^{-1}$ & $1.04 \times 10^{-1}$ & $3.50 \times 10^{-1}$ & $5.01 \times 10^{-1}$ & $5.19 \times 10^{-1}$ \\
\hline 564.19 & $1.38 \times 10^{-1}$ & $3.72 \times 10^{-1}$ & $4.91 \times 10^{-1}$ & $5.01 \times 10^{-1}$ & $1.04 \times 10^{-1}$ & $3.50 \times 10^{-1}$ & $5.01 \times 10^{-1}$ & $5.19 \times 10^{-1}$ \\
\hline \multicolumn{9}{|l|}{ Mn-54 } \\
\hline 0.56 & $3.79 \times 10^{-2}$ & $1.67 \times 10^{-1}$ & $3.90 \times 10^{-1}$ & $5.21 \times 10^{-1}$ & $1.05 \times 10^{-2}$ & $4.22 \times 10^{-2}$ & $7.89 \times 10^{-2}$ & $9.52 \times 10^{-2}$ \\
\hline 2.8 & $2.94 \times 10^{-1}$ & 1.27 & 2.68 & 3.16 & $2.63 \times 10^{-1}$ & 1.06 & 1.97 & 2.38 \\
\hline 5.6 & $4.72 \times 10^{-1}$ & 1.93 & 3.65 & 4.05 & $3.65 \times 10^{-1}$ & 1.46 & 2.74 & 3.31 \\
\hline 13 & $6.78 \times 10^{-1}$ & 2.46 & 4.19 & 4.52 & $5.39 \times 10^{-1}$ & 2.17 & 4.05 & 4.89 \\
\hline 20 & $7.63 \times 10^{-1}$ & 2.59 & 4.32 & 4.62 & $6.67 \times 10^{-1}$ & 2.68 & 5.00 & 6.04 \\
\hline 564.19 & $9.96 \times 10^{-1}$ & 2.83 & 4.59 & 5.16 & $6.67 \times 10^{-1}$ & 2.68 & 5.00 & 6.04 \\
\hline
\end{tabular}


TABLE 15 (Cont.)

\begin{tabular}{|c|c|c|c|c|c|c|c|c|}
\hline \multirow{2}{*}{$\begin{array}{c}\text { Radius } \\
(\mathrm{m})\end{array}$} & \multicolumn{4}{|c|}{ New Model } & \multicolumn{4}{|c|}{ Old Model } \\
\hline & $T s=1 \mathrm{~cm}$ & $T s=5 \mathrm{~cm}$ & $T s=15 \mathrm{~cm}$ & $T s=50 \mathrm{~cm}$ & $T s=1 \mathrm{~cm}$ & $T s=5 \mathrm{~cm}$ & $T s=15 \mathrm{~cm}$ & $T s=50 \mathrm{~cm}$ \\
\hline \multicolumn{9}{|l|}{ U-234 } \\
\hline 0.56 & $1.44 \times 10^{-5}$ & $3.64 \times 10^{-5}$ & $5.13 \times 10^{-5}$ & $5.17 \times 10^{-5}$ & $4.70 \times 10^{-6}$ & $1.53 \times 10^{-5}$ & $2.10 \times 10^{-5}$ & $2.16 \times 10^{-5}$ \\
\hline 2.8 & $8.91 \times 10^{-5}$ & $2.21 \times 10^{-4}$ & $2.71 \times 10^{-4}$ & $2.73 \times 10^{-4}$ & $1.18 \times 10^{-4}$ & $3.82 \times 10^{-4}$ & $5.26 \times 10^{-4}$ & $5.39 \times 10^{-4}$ \\
\hline 5.6 & $1.28 \times 10^{-4}$ & $2.86 \times 10^{-4}$ & $3.38 \times 10^{-4}$ & $3.40 \times 10^{-4}$ & $1.63 \times 10^{-4}$ & $5.30 \times 10^{-4}$ & $7.30 \times 10^{-4}$ & $7.49 \times 10^{-4}$ \\
\hline 13 & $1.62 \times 10^{-4}$ & $3.21 \times 10^{-4}$ & $3.68 \times 10^{-4}$ & $3.69 \times 10^{-4}$ & $2.41 \times 10^{-4}$ & $7.83 \times 10^{-4}$ & $1.08 \times 10^{-3}$ & $1.11 \times 10^{-3}$ \\
\hline 20 & $1.72 \times 10^{-4}$ & $3.28 \times 10^{-4}$ & $3.73 \times 10^{-4}$ & $3.75 \times 10^{-4}$ & $2.98 \times 10^{-4}$ & $9.68 \times 10^{-4}$ & $1.33 \times 10^{-3}$ & $1.37 \times 10^{-3}$ \\
\hline 564.19 & $1.89 \times 10^{-4}$ & $3.40 \times 10^{-4}$ & $3.99 \times 10^{-4}$ & $4.02 \times 10^{-4}$ & $2.98 \times 10^{-4}$ & $9.68 \times 10^{-4}$ & $1.33 \times 10^{-3}$ & $1.37 \times 10^{-3}$ \\
\hline \multicolumn{9}{|l|}{ U-235 } \\
\hline 0.56 & $6.28 \times 10^{-3}$ & $3.20 \times 10^{-2}$ & $7.25 \times 10^{-2}$ & $8.41 \times 10^{-2}$ & $2.93 \times 10^{-3}$ & $9.78 \times 10^{-3}$ & $1.39 \times 10^{-2}$ & $1.44 \times 10^{-2}$ \\
\hline 2.8 & $5.16 \times 10^{-2}$ & $2.55 \times 10^{-1}$ & $4.63 \times 10^{-1}$ & $4.91 \times 10^{-1}$ & $7.33 \times 10^{-2}$ & $2.45 \times 10^{-1}$ & $3.47 \times 10^{-1}$ & $3.59 \times 10^{-1}$ \\
\hline 5.6 & $8.62 \times 10^{-2}$ & $3.85 \times 10^{-1}$ & $6.02 \times 10^{-1}$ & $6.23 \times 10^{-1}$ & $1.02 \times 10^{-1}$ & $3.39 \times 10^{-1}$ & $4.82 \times 10^{-1}$ & $4.98 \times 10^{-1}$ \\
\hline 13 & $1.30 \times 10^{-1}$ & $4.70 \times 10^{-1}$ & $6.79 \times 10^{-1}$ & $6.86 \times 10^{-1}$ & $1.51 \times 10^{-1}$ & $5.02 \times 10^{-1}$ & $7.13 \times 10^{-1}$ & $7.37 \times 10^{-1}$ \\
\hline 20 & $1.48 \times 10^{-1}$ & $4.91 \times 10^{-1}$ & $6.96 \times 10^{-1}$ & $6.95 \times 10^{-1}$ & $1.86 \times 10^{-1}$ & $6.20 \times 10^{-1}$ & $8.82 \times 10^{-1}$ & $9.11 \times 10^{-1}$ \\
\hline 564.19 & $1.90 \times 10^{-1}$ & $5.24 \times 10^{-1}$ & $7.31 \times 10^{-1}$ & $7.57 \times 10^{-1}$ & $1.86 \times 10^{-1}$ & $6.20 \times 10^{-1}$ & $8.82 \times 10^{-1}$ & $9.11 \times 10^{-1}$ \\
\hline \multicolumn{9}{|l|}{ U-238 } \\
\hline 0.56 & $1.19 \times 10^{-3}$ & $5.12 \times 10^{-3}$ & $1.13 \times 10^{-2}$ & $1.43 \times 10^{-2}$ & $2.47 \times 10^{-4}$ & $9.69 \times 10^{-4}$ & $1.75 \times 10^{-3}$ & $2.04 \times 10^{-3}$ \\
\hline 2.8 & $9.50 \times 10^{-3}$ & $3.87 \times 10^{-2}$ & $7.43 \times 10^{-2}$ & $8.51 \times 10^{-2}$ & $6.17 \times 10^{-3}$ & $2.42 \times 10^{-2}$ & $4.37 \times 10^{-2}$ & $5.10 \times 10^{-2}$ \\
\hline 5.6 & $1.55 \times 10^{-2}$ & $5.74 \times 10^{-2}$ & $9.95 \times 10^{-2}$ & $1.09 \times 10^{-1}$ & $8.56 \times 10^{-3}$ & $3.36 \times 10^{-2}$ & $6.06 \times 10^{-2}$ & $7.08 \times 10^{-2}$ \\
\hline 13 & $2.25 \times 10^{-2}$ & $7.12 \times 10^{-2}$ & $1.14 \times 10^{-1}$ & $1.21 \times 10^{-1}$ & $1.27 \times 10^{-2}$ & $4.97 \times 10^{-2}$ & $8.96 \times 10^{-2}$ & $1.05 \times 10^{-1}$ \\
\hline 20 & $2.53 \times 10^{-2}$ & $7.46 \times 10^{-2}$ & $1.17 \times 10^{-1}$ & $1.23 \times 10^{-1}$ & $1.57 \times 10^{-2}$ & $6.15 \times 10^{-2}$ & $1.11 \times 10^{-1}$ & $1.29 \times 10^{-1}$ \\
\hline 564.19 & $3.21 \times 10^{-2}$ & $8.06 \times 10^{-2}$ & $1.24 \times 10^{-1}$ & $1.37 \times 10^{-1}$ & $1.57 \times 10^{-2}$ & $6.15 \times 10^{-2}$ & $1.11 \times 10^{-1}$ & $1.29 \times 10^{-1}$ \\
\hline \multicolumn{9}{|l|}{ Al-26 } \\
\hline 0.56 & $1.12 \times 10^{-1}$ & $5.12 \times 10^{-1}$ & 1.23 & 1.71 & $3.22 \times 10^{-2}$ & $1.32 \times 10^{-1}$ & $2.57 \times 10^{-1}$ & $3.23 \times 10^{-1}$ \\
\hline 2.8 & $8.75 \times 10^{-1}$ & 3.88 & 8.55 & $1.06 \times 10^{1}$ & $8.04 \times 10^{-1}$ & 3.30 & 6.42 & 8.07 \\
\hline 5.6 & 1.40 & 5.92 & $1.18 \times 10^{1}$ & $1.37 \times 10^{1}$ & 1.12 & 4.58 & 8.91 & $1.12 \times 10^{1}$ \\
\hline 13 & 2.02 & 7.60 & $1.37 \times 10^{1}$ & $1.53 \times 10^{1}$ & 1.65 & 6.77 & $1.32 \times 10^{1}$ & $1.66 \times 10^{1}$ \\
\hline 20 & 2.27 & 8.03 & $1.41 \times 10^{1}$ & $1.56 \times 10^{1}$ & 2.04 & 8.37 & $1.63 \times 10^{1}$ & $2.05 \times 10^{1}$ \\
\hline 564.19 & 3.01 & 8.81 & $1.50 \times 10^{1}$ & $1.73 \times 10^{1}$ & 2.04 & 8.37 & $1.63 \times 10^{1}$ & $2.05 \times 10^{1}$ \\
\hline
\end{tabular}




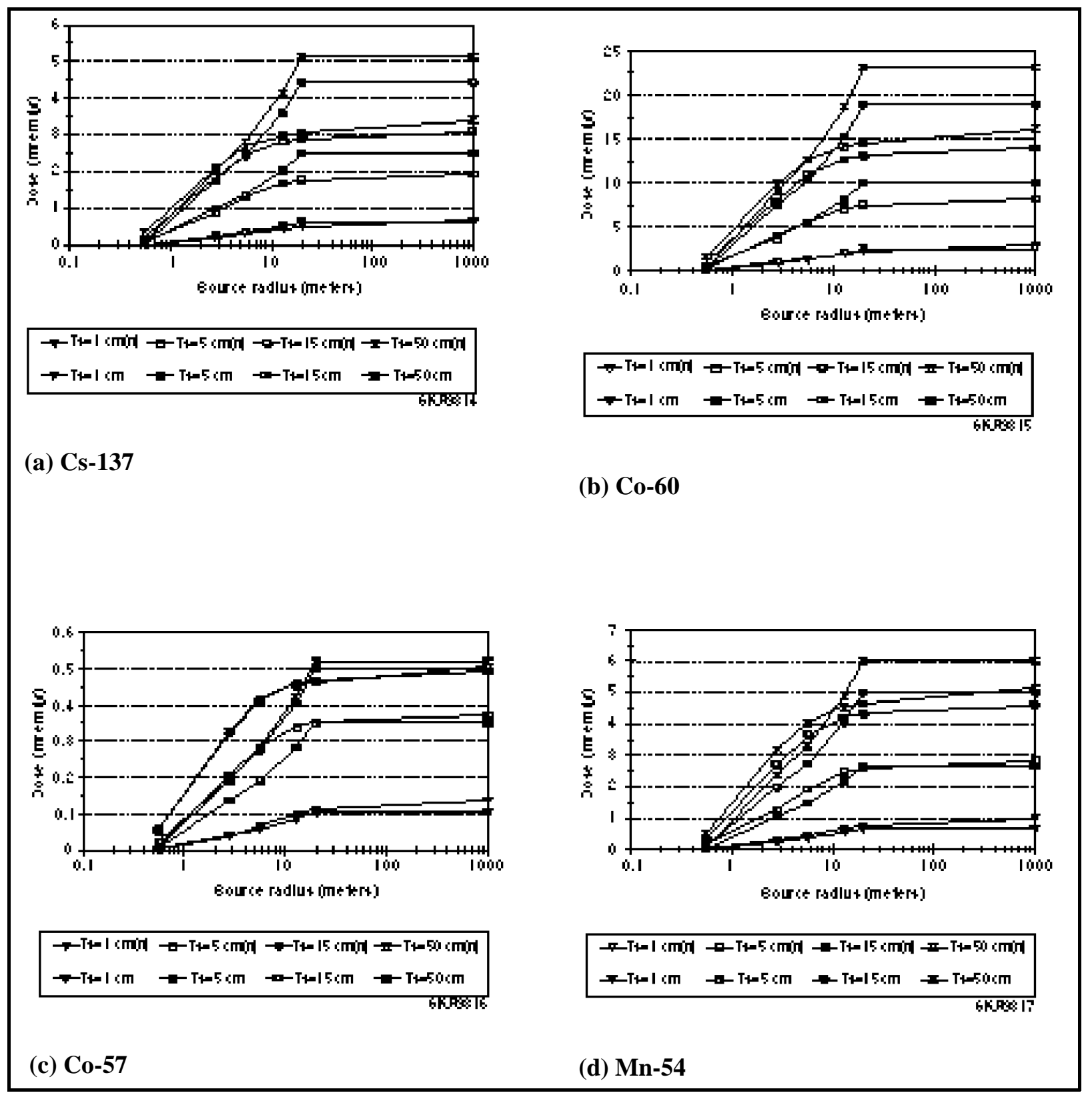

FIGURE 8 Dose Comparison as a Function of Source Radius for a Set of Source Depths with No Cover (Depths: 1 to $50 \mathrm{~cm}$ ) (Note: (n) in legends denotes results for new RESRAD model.) 

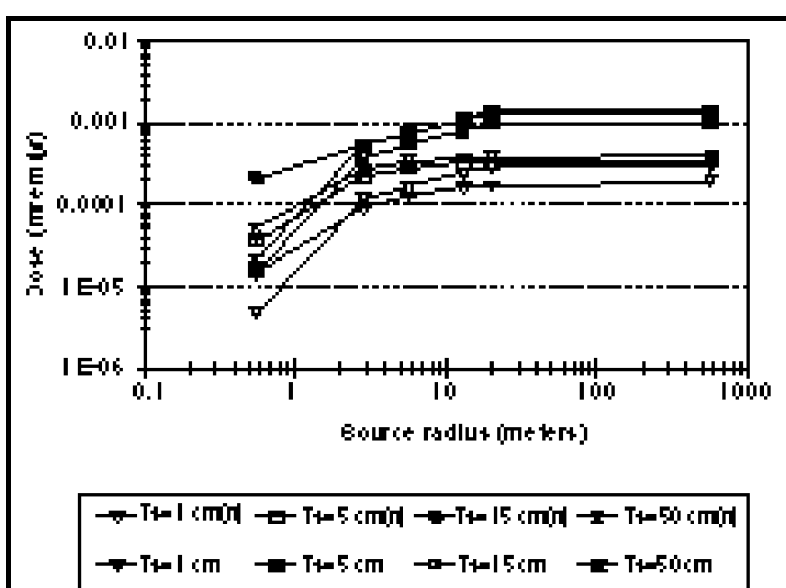

GFAs IS

(e) U-234
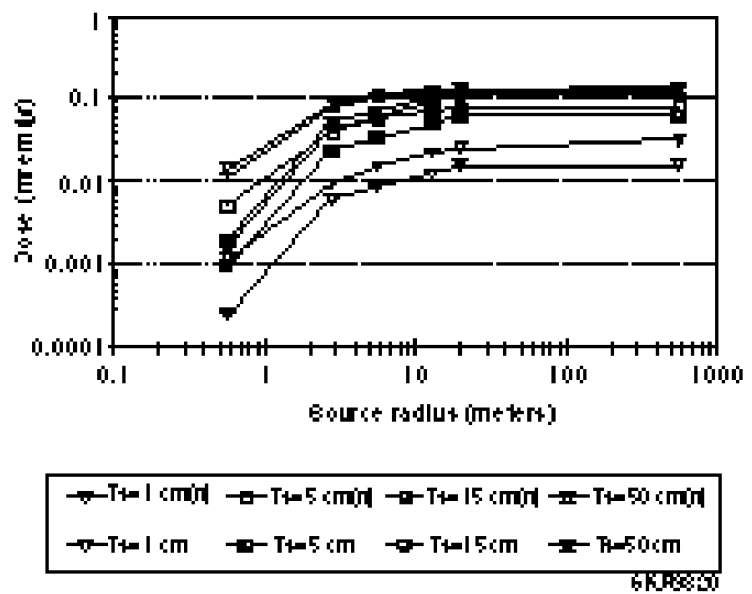

(g) U-238
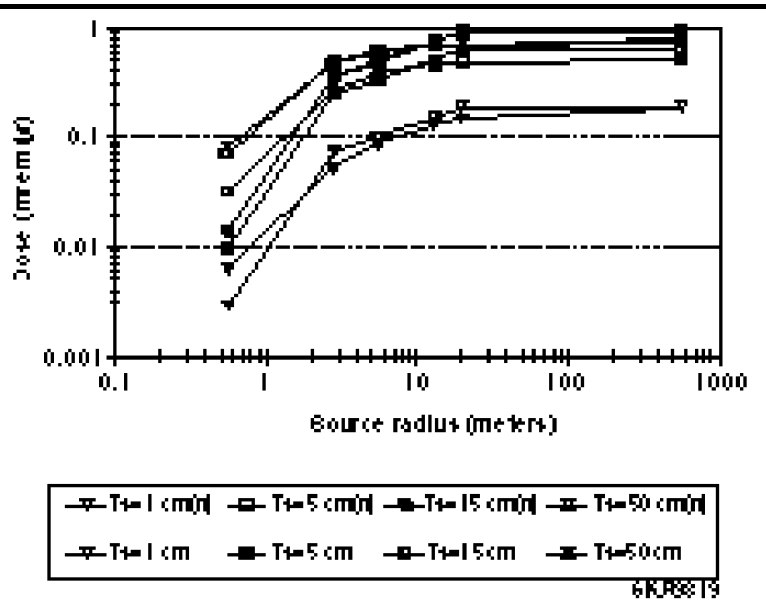

(f) U-235
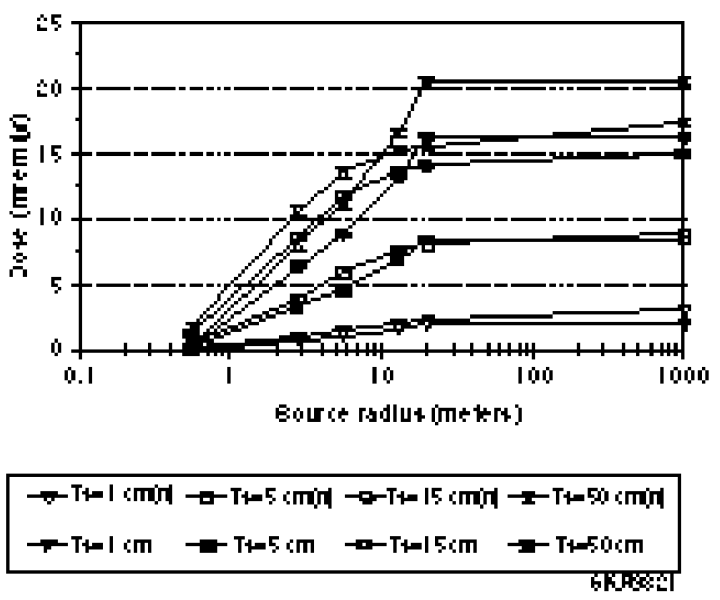

(h) Al-26

\section{FIGURE 8 (Cont.)}


Figure 8c compares Co-57 doses; in both models the values are the same, and differences only occur because of depth and area factors. For Co-57, at a small radius (under $13 \mathrm{~m}$ ), the new model always gives higher dose values. For thick sources with a radius over $13 \mathrm{~m}$, values are close in the two models.

Table 16 and Figure 9 give the dose comparison at four radii $(0.56,2.8,13.0$, and $564.19 \mathrm{~m})$ for Co-60 source as a function of source depth. Table 17 gives the dose comparison at four radii for Co-60 source as a function of cover thickness for a source depth of $50 \mathrm{~cm}$; Figure 9 shows the results.

TABLE 16 Dose (mrem/yr) Comparison for Co-60 as a Function of Source Depth at Different Radii $(0.56,2.8,13.0$, and 564.19 m) for New and Old RESRAD Models

\begin{tabular}{|c|c|c|c|c|c|c|c|c|}
\hline \multirow{2}{*}{$\begin{array}{c}\text { Source } \\
\text { Depth } \\
(\mathrm{cm})\end{array}$} & \multicolumn{4}{|c|}{ New Model } & \multicolumn{4}{|c|}{ Old Model } \\
\hline & $r=0.56 \mathrm{~m}$ & $r=2.8 \mathrm{~m}$ & $r=13.0 \mathrm{~m}$ & $r=564.19 \mathrm{~m}$ & $r=0.56 \mathrm{~m}$ & $r=2.8 \mathrm{~m}$ & $r=13.0 \mathrm{~m}$ & $r=564.19 \mathrm{~m}$ \\
\hline 1.0 & $1.08 \times 10^{-1}$ & $8.33 \times 10^{-1}$ & 1.91 & 2.83 & $3.89 \times 10^{-2}$ & $9.74 \times 10^{-1}$ & 2.00 & 2.47 \\
\hline 2.0 & $2.01 \times 10^{-1}$ & 1.55 & 3.41 & 4.53 & $7.37 \times 10^{-2}$ & 1.84 & 3.78 & 4.68 \\
\hline 3.0 & $2.89 \times 10^{-1}$ & 2.23 & 4.68 & 5.92 & $1.05 \times 10^{-1}$ & 2.62 & 5.38 & 6.65 \\
\hline 5.0 & $4.76 \times 10^{-1}$ & 3.64 & 7.10 & 8.20 & $1.57 \times 10^{-1}$ & 3.94 & 8.08 & 9.99 \\
\hline 10.0 & $8.41 \times 10^{-1}$ & 6.09 & $1.06 \times 10^{1}$ & $1.19 \times 10^{1}$ & $2.47 \times 10^{-1}$ & 6.18 & $1.27 \times 10^{1}$ & $1.57 \times 10^{1}$ \\
\hline 15.0 & 1.16 & 8.02 & $1.27 \times 10^{1}$ & $1.39 \times 10^{1}$ & $2.98 \times 10^{-1}$ & 7.46 & $1.53 \times 10^{1}$ & $1.89 \times 10^{1}$ \\
\hline 25.0 & 1.36 & 9.12 & $1.40 \times 10^{1}$ & $1.55 \times 10^{1}$ & $3.44 \times 10^{-1}$ & 8.60 & $1.77 \times 10^{1}$ & $2.18 \times 10^{1}$ \\
\hline 35.0 & 1.48 & 9.55 & $1.43 \times 10^{1}$ & $1.60 \times 10^{1}$ & $3.59 \times 10^{-1}$ & 8.97 & $1.84 \times 10^{1}$ & $2.28 \times 10^{1}$ \\
\hline 50.0 & 1.60 & 9.88 & $1.42 \times 10^{1}$ & $1.62 \times 10^{1}$ & $3.65 \times 10^{-1}$ & 9.12 & $1.87 \times 10^{1}$ & $2.31 \times 10^{1}$ \\
\hline
\end{tabular}

TABLE 17 Dose (mrem/yr) Comparison for Co-60 (50-cm depth) as a Function of Cover Thickness at Different Radii $(0.56,2.8,13.0$, and 564.19 m) for New and Old RESRAD Models

\begin{tabular}{|c|c|c|c|c|c|c|c|c|}
\hline \multirow{2}{*}{$\begin{array}{c}\text { Cover } \\
\text { Thick- } \\
\text { ness } \\
(\mathrm{cm})\end{array}$} & \multicolumn{4}{|c|}{ New Model } & \multicolumn{4}{|c|}{ Old Model } \\
\hline & $r=0.56 \mathrm{~m}$ & $r=2.8 \mathrm{~m}$ & $r=13.0 \mathrm{~m}$ & $r=564.19 \mathrm{~m}$ & $r=0.56 \mathrm{~m}$ & $r=2.8 \mathrm{~m}$ & $r=13.0 \mathrm{~m}$ & $r=564.19 \mathrm{~m}$ \\
\hline 0.0 & 1.60 & 9.88 & $1.42 \times 10^{1}$ & $1.62 \times 10^{1}$ & $3.65 \times 10^{-1}$ & 9.12 & $1.87 \times 10^{1}$ & $2.31 \times 10^{1}$ \\
\hline 0.5 & 1.62 & 9.85 & $1.38 \times 10^{1}$ & $1.45 \times 10^{1}$ & $3.45 \times 10^{-1}$ & 8.62 & $1.77 \times 10^{1}$ & $2.19 \times 10^{1}$ \\
\hline 1.0 & 1.57 & 9.45 & $1.30 \times 10^{1}$ & $1.34 \times 10^{1}$ & $3.26 \times 10^{-1}$ & 8.15 & $1.67 \times 10^{1}$ & $2.07 \times 10^{1}$ \\
\hline 2.0 & 1.48 & 8.76 & $1.15 \times 10^{1}$ & $1.17 \times 10^{1}$ & $2.91 \times 10^{-1}$ & 7.28 & $1.49 \times 10^{1}$ & $1.85 \times 10^{1}$ \\
\hline 3.0 & 1.38 & 8.01 & $1.02 \times 10^{1}$ & $1.03 \times 10^{1}$ & $2.60 \times 10^{-1}$ & 6.51 & $1.34 \times 10^{1}$ & $1.65 \times 10^{1}$ \\
\hline 5.0 & 1.20 & 6.70 & 7.99 & 7.99 & $2.08 \times 10^{-1}$ & 5.20 & $1.07 \times 10^{1}$ & $1.32 \times 10^{1}$ \\
\hline 10.0 & $7.60 \times 10^{-1}$ & 3.83 & 4.27 & 4.27 & $1.18 \times 10^{-1}$ & 2.96 & 6.08 & 7.51 \\
\hline 15.0 & $4.70 \times 10^{-1}$ & 2.18 & 2.28 & 2.28 & $6.75 \times 10^{-2}$ & 1.69 & 3.46 & 4.28 \\
\hline 20.0 & $2.66 \times 10^{-1}$ & 1.17 & 1.22 & 1.22 & $3.84 \times 10^{-2}$ & $9.61 \times 10^{-1}$ & 1.97 & 2.44 \\
\hline 25.0 & $1.51 \times 10^{-1}$ & $6.30 \times 10^{-1}$ & $6.52 \times 10^{-1}$ & $6.52 \times 10^{-1}$ & $2.97 \times 10^{-2}$ & $5.48 \times 10^{-1}$ & 1.12 & 1.39 \\
\hline
\end{tabular}




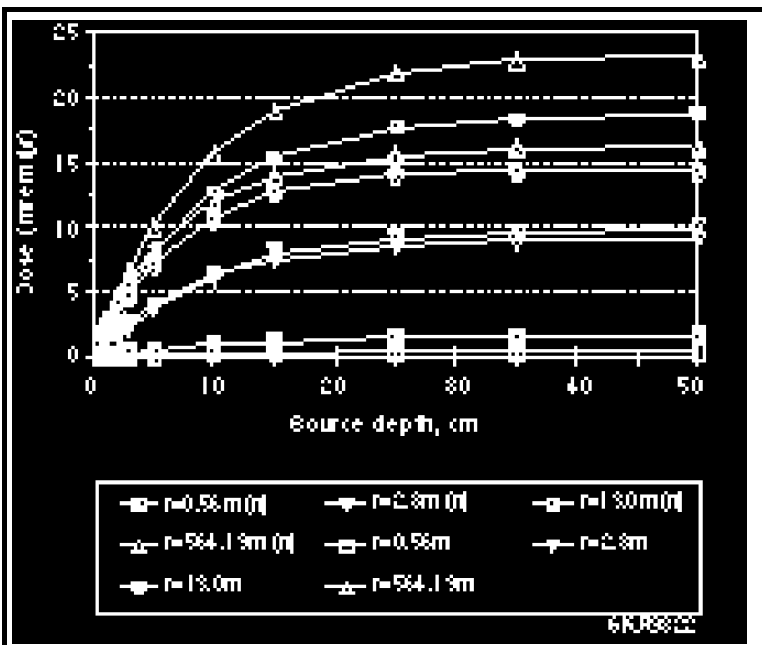

(a) Actual Value at Different Source Depths (No Cover)

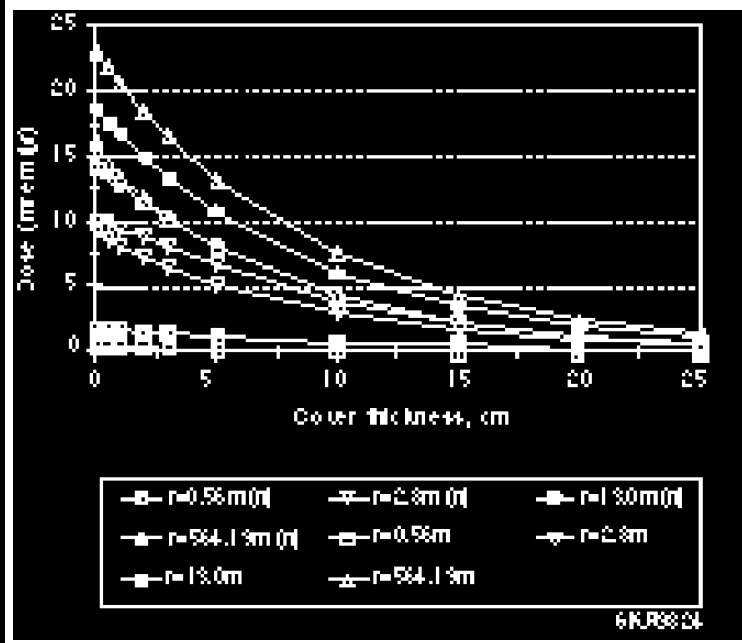

(c) Dose Variation with Cover Thickness (Source Depth $=50 \mathrm{~cm})$

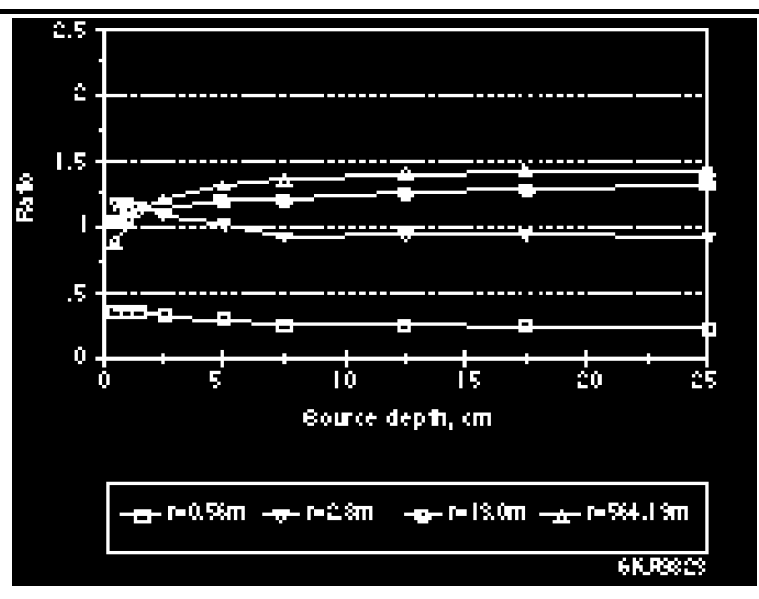

(b) Ratio at Different Source Depths (No Cover)

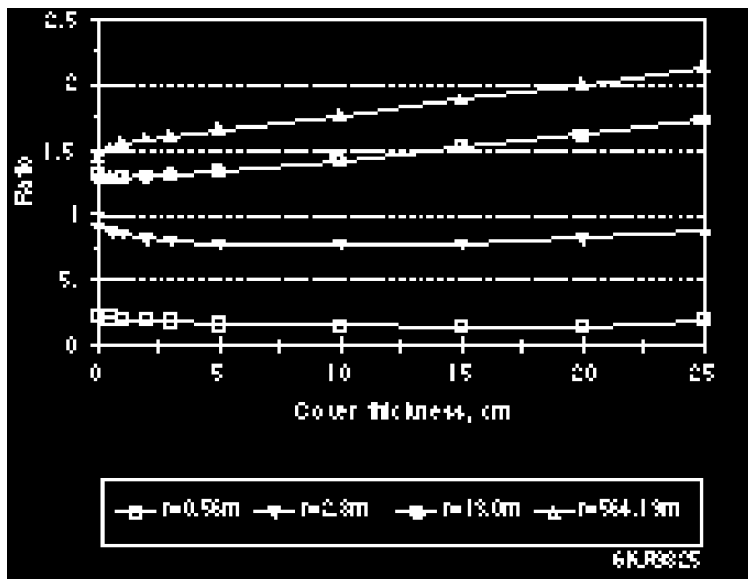

(d) Ratio at Different Cover Thicknesses (Source Depth $=\mathbf{5 0} \mathrm{cm}$ )

FIGURE 9 Dose Comparison of Old and New RESRAD Models as a Function of Various Dependent Parameters for a Set of Co-60-Contaminated Source Radii (Note: (n) in legends denotes results for new RESRAD model.) 


\section{CONCLUSIONS}

A new external exposure model, based on dose conversion factors from FGR-12 (Eckerman and Ryman 1993) and the point kernel method, has been developed for use in the RESRAD code. This model improves the external ground pathway dose estimation from the earlier version of the RESRAD code by extending FGR-12 data applicability to a wider range of source geometries. FGR-12 provides the dose coefficients for external exposure to photons and electrons emitted by radionuclides distributed in soil; tabulated values are given for surface and uniformly distributed volume sources at four specific thicknesses $(1,5$, and $15 \mathrm{~cm}$ and effectively infinite) of soil with a density of $1.6 \mathrm{~g} / \mathrm{cm}^{3}$.

Differences in the calculated doses between the old and new RESRAD models arise because of differences in the dose conversion coefficients between FGR-12 and the old RESRAD, differences in the cover-and-depth factor in the two methods, and differences in the area factor. The area factor in the new model depends on energy, source area, source depth, and cover thickness. The old RESRAD model assumed that area factor is a function of area only. In addition to the differences in DCFs, the major differences between the old and new models will occur for small energies, small source radii, small source depths, and small cover thicknesses.

Comparison of the derived depth factor function with FGR-12 values shows that the factors are within $2 \%$ for all depths for most radionuclides. The cover-and-depth factor comparison shows that for small cover thicknesses, most values are within $2 \%$, and for large covers $(5 \mathrm{~cm}, 15 \mathrm{~cm})$, most values are within $10 \%$. Comparison of the old and new RESRAD model depth factors shows no significant difference at depths greater than $30 \mathrm{~cm}$, but major differences occur for thin sources. The new model gives higher depth factors for thin sources, which means that the new model will predict higher doses for thin sources. The cover-and-depth factor comparisons show large differences for thin sources without any cover and for any sources with very thick covers. For all source depths with covers between 0.5 and $10 \mathrm{~cm}$, the ratio of the cover-and-depth factor of the old to new RESRAD models is within 0.8 and 1.2.

The area factor increases with the source radius and approaches unity for a radius greater than $50 \mathrm{~m}$. The area factor decreases with increasing energy, with sharp decreases when the energy changes from 10 to $60 \mathrm{keV}$ and slight variations when the energy is higher. The area factor also increases with increasing source depth. The area factors of sources with depths greater than $10 \mathrm{~cm}$ were always greater in the new model. For very thin sources $(0.1-\mathrm{cm}$ depth) the old RESRAD model values are higher except for energies below $30 \mathrm{keV}$, for which the new model gives higher values. Values compare reasonably well in the two models at a source depth of $10 \mathrm{~cm}$ for energies above $30 \mathrm{keV}$. 


\section{REFERENCES}

Briesmeister, J.F. (editor), 1993, MCNP - A General Monte Carlo N-Particle Transport Code, Version 4A, LA-12625, Los Alamos National Laboratory, Los Alamos, N.M.

Chen, S.Y., 1991, "Calculation of Effective Dose Equivalent Responses for External Exposure from Residual Photon Emitters in Soil,” Health Physics 60:411-426.

Cristy, M., and K.F. Eckerman, 1987, Specific Absorbed Fractions of Energy at Various Ages from Internal Photon Sources, I. Methods, ORNL/TM-8381/VI, Oak Ridge National Laboratory, Oak Ridge, Tenn.

DOE: See U.S. Department of Energy.

Eckerman, K.F., and J.C. Ryman, 1993, External Exposure to Radionuclides in Air, Water, and Soil, Exposure to Dose Coefficients for General Application, Based on the 1987 Federal Radiation Protection Guidance, Federal Guidance Report No. 12, EPA 402-R-93-081, prepared by Oak Ridge National Laboratory, Oak Ridge, Tenn., for U.S. Environmental Protection Agency, Office of Radiation and Indoor Air, Washington, D.C.

EPA: See U.S. Environmental Protection Agency.

ICRP: See International Commission on Radiological Protection.

International Commission on Radiological Protection, 1983, Radionuclide Transformations: Energy and Intensity of Emissions, ICRP Publication 38, Annals of the ICRP, Vols. 11-13, Pergamon Press, New York, N.Y.

Kocher, D.C., and A.L. Sjoreen, 1985, "Dose-Rate Conversion Factors for External Exposure to Photon Emitters in Soil," Health Physics 28:193-205.

Napier, B.A., et al., 1984, Intruder Dose Pathway Analysis for the Onsite Disposal of Radioactive Wastes: The ONSITE/MAxI1 Computer Program, NUREG/CR-3620, PNL-4054, prepared by Pacific Northwest Laboratory, Richland, Wash., for U.S. Nuclear Regulatory Commission, Division of Waste Management, Washington, D.C.

Trubey, D.K., 1991, New Gamma-Ray Buildup Factor Data for Point Kernel Calculations: ANS-6.4.3 Standard Reference Data, NUREG-5740, ORNL/RSIC-49, prepared by Oak Ridge National Laboratory, Oak Ridge, Tenn., for U.S. Nuclear Regulatory Commission, Washington, D.C., Aug. 
U.S. Department of Energy, 1988, External Dose-Rate Conversion Factors for Calculation of Dose to the Public, DOE/EH-0070, Assistant Secretary for Environment, Safety and Health, Washington, D.C.

U.S. Environmental Protection Agency, 1987, "Radiation Protection Guidance to Federal Agencies for Occupational Exposure," Federal Register 52(17):2822; with corrections published in the Federal Register on Friday, Jan. 30, and Wednesday, Feb. 4, 1987.

Yu, C., et al., 1993a, Manual for Implementing Residual Radioactive Material Guidelines Using RESRAD, Version 5.0, Draft, ANL/EAD/LD-2, Argonne National Laboratory, Argonne, Ill., Sept.

Yu, C., et al., 1993b, Data Collection Handbook to Support Modeling the Impacts of Radioactive Material in Soil, ANL/EAIS-8, Argonne National Laboratory, Argonne, Ill. 


\section{APPENDIX:}

EXTERNAL EFFECTIVE DOSE EQUIVALENT CALCULATIONS FOR CONTAMINATED SOIL WITH THE MONTE CARLO N-PARTICLE TRANSPORT CODE 


\section{APPENDIX:}

\section{EXTERNAL EFFECTIVE DOSE EQUIVALENT CALCULATIONS FOR CONTAMINATED SOIL WITH THE MONTE CARLO N-PARTICLE TRANSPORT CODE}

The Monte Carlo N-Particle (MCNP) Transport Code (Briesmeister 1993) is used to calculate the external effective dose equivalent at a distance of $1 \mathrm{~m}$ from contaminated soil of varying thicknesses. The calculated results are then compared with values from the Federal Guidance Report No. 12 (FGR-12) (Eckerman and Ryman 1993) for different isotopes.

\section{A.1 MCNP CALCULATIONS}

MCNP is a general-purpose, continuous energy, generalized geometry, time-dependent code that can be used for neutron, photon, electron, or coupled neutron/photon/electron transport. The photon and electron energy regimes are from $1 \mathrm{keV}$ to $1,000 \mathrm{MeV}$. Photon interaction tables exist for all elements from atomic numbers 1 through 94. The data in the photon interaction tables allow MCNP to account for coherent and incoherent scattering, photoelectric absorption and the possibility of fluorescent emission, and pair production. Scattering angular distributions are modified by atomic form factors and incoherent scattering functions. To run a problem, an input file is prepared that contains such information as the geometry specification, the description of the materials and selection of cross-section evaluations, the location and characteristics of the source, the type of answers or tallies desired, and variance reduction techniques used to improve efficiency.

For MCNP calculations, gamma energies and their respective abundances were taken from the International Commission on Radiological Protection (ICRP) Publication 38 (ICRP 1983) (Table A.1). Flux is calculated at a height of $100 \mathrm{~cm}$ from the contaminated source by using a point detector next-event estimator. A point detector is known as a "next event estimator" because it is a tally of the flux at a point if the next event is a trajectory directly to the point detector without further collision. A point detector is a deterministic estimate (from the current event point) of the flux at a point in space. The contributions to the point detector are made at source and collision events throughout the random walk. Flux at the detector is given by:

$$
\begin{aligned}
\mathrm{Q}(r, E, t, \mu) & \frac{W p(\mu) e^{\S 8}}{2 \mathrm{~B} R^{2}} \text { and } \\
8{ }^{\prime} & \mathrm{m}_{0}^{R} \mathrm{E}_{t}(s) d s,
\end{aligned}
$$


TABLE A.1 Gamma Energies and Yield

\begin{tabular}{|c|c|c|}
\hline Isotope & Energy (keV) & $\begin{array}{c}\text { Yield } \\
(\%)\end{array}$ \\
\hline \multirow[t]{2}{*}{ Co-60 } & $1.173 \times 10^{3}$ & $1.00 \times 10^{2}$ \\
\hline & $1.332 \times 10^{3}$ & $1.00 \times 10^{2}$ \\
\hline Mn-54 & $8.348 \times 10^{2}$ & $1.00 \times 10^{2}$ \\
\hline \multirow[t]{10}{*}{ Ra-226 } & $2.623 \times 10^{2}$ & $5.40 \times 10^{-3}$ \\
\hline & $1.860 \times 10^{2}$ & 3.28 \\
\hline & $9.771 \times 10^{1}$ & $3.36 \times 10^{-2}$ \\
\hline & $9.487 \times 10^{1}$ & $6.79 \times 10^{-2}$ \\
\hline & $9.424 \times 10^{1}$ & $3.55 \times 10^{-2}$ \\
\hline & $8.378 \times 10^{1}$ & $2.98 \times 10^{-1}$ \\
\hline & $8.107 \times 10^{1}$ & $1.79 \times 10^{-1}$ \\
\hline & $1.688 \times 10^{1}$ & $9.04 \times 10^{-2}$ \\
\hline & $1.428 \times 10^{1}$ & $4.05 \times 10^{-1}$ \\
\hline & $1.171 \times 10^{1}$ & $2.53 \times 10^{-1}$ \\
\hline \multirow[t]{6}{*}{ U-238 } & $4.955 \times 10^{1}$ & $6.79 \times 10^{-2}$ \\
\hline & $1.909 \times 10^{1}$ & 1.02 \\
\hline & $1.610 \times 10^{1}$ & 4.47 \\
\hline & $1.451 \times 10^{1}$ & $9.20 \times 10^{-2}$ \\
\hline & $1.295 \times 10^{1}$ & 2.96 \\
\hline & $1.112 \times 10^{1}$ & $1.41 \times 10^{-1}$ \\
\hline \multirow[t]{4}{*}{ Al-26 } & $5.110 \times 10^{2}$ & $1.64 \times 10^{2}$ \\
\hline & $1.130 \times 10^{3}$ & 2.50 \\
\hline & $1.809 \times 10^{3}$ & $9.98 \times 10^{1}$ \\
\hline & $2.938 \times 10^{3}$ & $2.40 \times 10^{-1}$ \\
\hline
\end{tabular}

Source: ICRP (1983).

where

$$
\begin{aligned}
W= & \text { particle weight, } \\
8= & \begin{array}{l}
\text { total number of mean free paths integrated over the trajectory from the } \\
\text { source or collision point to the detector, }
\end{array} \\
s= & \begin{array}{l}
\text { measured distance along the direction from the collision or source point } \\
\\
\text { to the detector, }
\end{array} \\
\mathrm{E}_{t}(s)= & \text { macroscopic total cross section at } s,
\end{aligned}
$$




$$
\begin{aligned}
R= & \text { distance from source or collision event to detector, and } \\
p(\mu)= & \text { value of probability density function at } \mu \text {, the cosine of the angle } \\
& \text { between the particle trajectory and the direction to the detector. }
\end{aligned}
$$

The $e^{-8}$ term accounts for attenuation between the present event and the detector point. The $1 / 2 \mathrm{~B} R^{2}$ term accounts for the solid angle effect. The $p(\mu)$ term accounts for the probability of scattering toward the detector instead of in the direction selected in the random walk. Each contribution to the detector can be thought of as the transport of a pseudoparticle to the detector.

This flux is used to calculate the effective dose equivalent by using the conversion coefficients between effective dose equivalent and fluence values (Table A.2). Table A.2 lists conversion coefficients for anteroposterior (AP), posteroanterior (PA), lateral (LAT), rotational (ROT), and isotropic (ISO) geometries. For the calculations, ROT symmetry is assumed. These values are taken from ICRP Publication 51 (ICRP 1987).

For MCNP computations, cylindrical sources with a 10,000-cm radius and of different thicknesses were used for flux estimation. Soil and air composition was taken from FGR-12 (Eckerman and Ryman 1993). These values are shown in Tables A.3 and A.4, respectively. A soil density of $1.6 \mathrm{~g} / \mathrm{cm}^{3}$ was used.

Results obtained with MCNP are in units of Sv/gamma. These values were multiplied by gamma abundance and source volume to get the effective dose equivalent in units of $(\mathrm{Sv} / \mathrm{s}) /\left(\mathrm{Bq} / \mathrm{m}^{3}\right)$ for volume sources and were multiplied by gamma abundance and surface area to get the effective dose equivalent in units of $(\mathrm{Sv} / \mathrm{s}) /\left(\mathrm{Bq} / \mathrm{m}^{2}\right)$ for surface sources.

\section{A.2 COMPARISON OF FGR-12 AND MCNP RESULTS}

Effective dose equivalents for source depths of 1, 5, 15, 50, and $100 \mathrm{~cm}$ calculated with MCNP are compared with FGR values for Co-60, Mn-54, Ra-226, U-238, and Al-26 in Table A.5. Source sizes used in the MCNP calculations are also shown. Table A.6 gives the surface dose comparison.

Figure A.1 compares FGR-12 dose values with MCNP calculations for Co-60, Mn-54, Ra-226, U-238, and Al-26 at different source thicknesses. Figure A.2 compares the values for surface sources. A comparison of the volume sources shows that the ratio in all cases is between 0.93 and 1.10, and in most cases it is between 0.95 and 1.05. However, for the surface sources, FGR-12 results are always higher. One reason for this difference could be beta activity, which is not taken into account in MCNP calculations. Statistical uncertainty in MCNP calculations is less than 5\% at $1 \mathrm{~F}$. 
TABLE A.2 Effective Dose Equivalent per Unit Fluence for Photons Incident in Various Geometries on an Anthropomorphic Phantom

\begin{tabular}{|c|c|c|c|c|c|}
\hline \multirow{2}{*}{$\begin{array}{l}\text { Photon } \\
\text { Energy } \\
(\mathrm{MeV})\end{array}$} & \multicolumn{5}{|c|}{ Conversion Coefficient ${ }^{\mathrm{a}}\left(10^{-12} \mathrm{~Sv} \mathrm{~cm}{ }^{2}\right)$} \\
\hline & AP & PA & LAT & ROT & ISO \\
\hline 0.010 & 0.062 & 0.0 & 0.02 & 0.029 & 0.022 \\
\hline 0.015 & 0.157 & 0.0310 & 0.0330 & 0.0710 & 0.0570 \\
\hline 0.020 & 0.238 & 0.0868 & 0.0491 & 0.110 & 0.0912 \\
\hline 0.030 & 0.329 & 0.161 & 0.0863 & 0.166 & 0.138 \\
\hline 0.040 & 0.365 & 0.222 & 0.123 & 0.199 & 0.163 \\
\hline 0.050 & 0.384 & 0.260 & 0.152 & 0.222 & 0.180 \\
\hline 0.060 & 0.400 & 0.286 & 0.170 & 0.240 & 0.196 \\
\hline 0.080 & 0.451 & 0.344 & 0.212 & 0.293 & 0.237 \\
\hline 0.100 & 0.533 & 0.418 & 0.258 & 0.357 & 0.284 \\
\hline 0.150 & 0.777 & 0.624 & 0.396 & 0.534 & 0.436 \\
\hline 0.200 & 1.03 & 0.844 & 0.557 & 0.731 & 0.602 \\
\hline 0.300 & 1.56 & 1.30 & 0.891 & 1.14 & 0.949 \\
\hline 0.400 & 2.06 & 1.76 & 1.24 & 1.55 & 1.30 \\
\hline 0.500 & 2.54 & 2.20 & 1.58 & 1.96 & 1.64 \\
\hline 0.600 & 2.99 & 2.62 & 1.92 & 2.34 & 1.98 \\
\hline 0.800 & 3.83 & 3.43 & 2.60 & 3.07 & 2.64 \\
\hline 1.000 & 4.60 & 4.18 & 3.24 & 3.75 & 3.27 \\
\hline 1.500 & 6.24 & 5.80 & 4.70 & 5.24 & 4.68 \\
\hline 2.000 & 7.66 & 7.21 & 6.02 & 6.56 & 5.93 \\
\hline 3.000 & 10.2 & 9.71 & 8.40 & 8.90 & 8.19 \\
\hline 4.000 & 12.5 & 12.0 & 10.6 & 11.0 & 10.2 \\
\hline 5.000 & 14.7 & 14.1 & 12.6 & 13.0 & 12.1 \\
\hline 6.000 & 16.7 & 16.2 & 14.6 & 14.9 & 14.0 \\
\hline 8.000 & 20.8 & 20.2 & 18.5 & 18.9 & 17.8 \\
\hline 10.00 & 24.7 & 24.2 & 22.3 & 22.9 & 21.6 \\
\hline
\end{tabular}

a The geometries are as follows: $\mathrm{AP}=$ anteroposterior, $\mathrm{PA}=$ posteroanterior, $\mathrm{LAT}=$ lateral, $\mathrm{ROT}=$ rotational, $\mathrm{ISO}=$ isotropic.

Source: ICRP (1987). 


\section{A.3 COMPARISON OF COVER-AND-DEPTH FACTOR WITH MCNP}

Doses were compared between MCNP and the new RESRAD model for different source depths and cover thicknesses for Co-60 and Mn-54. These two radionuclides were chosen because of their relatively simple decay. Table A.7 gives the source depth factor comparison. Source depths were varied from 0.5 to $100.0 \mathrm{~cm}$.

Figure A.3 shows the depth factor comparison of the new model with MCNP calculations. The ratio of new model to MCNP varies between 1.12 and 0.93 ; in most cases, differences are less than $7 \%$.

Table A.8 gives the cover factor comparison for source depths of $1,5,15$, and $50 \mathrm{~cm}$. The cover thickness varies from 0.0 to $25.0 \mathrm{~cm}$. These results are shown in Figure A.4.

Table A.9 and Figure A.5 show the ratio of the new model cover-and-depth factor results compared with MCNP calculations for Co-60 and Mn-54. Comparisons were made for source depths of $1,5,15$, and $50 \mathrm{~cm}$.

Tables A.8 and A.9 and Figures A.4 and A.5
TABLE A.3 Soil Composition

\begin{tabular}{lc}
\hline Element & Mass Fraction \\
\hline & \\
$\mathrm{H}$ & 0.021 \\
$\mathrm{C}$ & 0.016 \\
$\mathrm{O}$ & 0.577 \\
$\mathrm{Al}$ & 0.050 \\
$\mathrm{Si}$ & 0.271 \\
$\mathrm{~K}$ & 0.013 \\
$\mathrm{Ca}$ & 0.041 \\
$\mathrm{Fe}$ & 0.011 \\
Total & 1.000 \\
\hline
\end{tabular}

Source: Eckerman and Ryman (1993).

\begin{tabular}{lc}
\hline Element & Mass Fraction \\
\hline $\mathrm{H}$ & 0.00064 \\
$\mathrm{C}$ & 0.00014 \\
$\mathrm{~N}$ & 0.75086 \\
$\mathrm{O}$ & 0.23555 \\
$\mathrm{Ar}$ & 0.01281 \\
Total & 1.00000 \\
\hline
\end{tabular}

Source: Eckerman and Ryman (1993). show that the ratios of new model to MCNP values are close to unity for small cover thicknesses (less than $25 \mathrm{~cm}$ ). Large differences are observed for cover thicknesses greater than $10 \mathrm{~cm}$ and at source depths greater than $15 \mathrm{~cm}$. For cover thicknesses greater than $10 \mathrm{~cm}$ at source depths greater than $15 \mathrm{~cm}$, the resultant dose, which is calculated as the difference between the doses for sources greater than $25 \mathrm{~cm}$ deep and sources greater than $15 \mathrm{~cm}$ deep, is small. As one example, for a Co-60 source at a depth of $15 \mathrm{~cm}$ without any cover, the dose is $13.9 \mathrm{mrem} / \mathrm{yr}$ (Table A.8), but the dose decreases to $0.55 \mathrm{mrem} / \mathrm{yr}$ with $25 \mathrm{~cm}$ of cover (a decrease by a factor of more than 20). Although the absolute difference between the two results (the MCNP and the new RESRAD model) is small (0.91 vs. $0.55 \mathrm{mrem} / \mathrm{yr}$, Table A.8). the relative difference can appear large; that is, the uncertainty factor is amplified. However, because the doses are so small for these conditions, the differences between the model results are not of significant concern. 
TABLE A.5 Comparison of MCNP and FGR-12 Dose Conversion Factors for Various Radionuclides

\begin{tabular}{|c|c|c|c|c|c|c|}
\hline \multirow[b]{2}{*}{ Isotope } & \multirow{2}{*}{$\begin{array}{c}\text { Thickness } \\
(\mathrm{cm})\end{array}$} & \multirow{2}{*}{$\begin{array}{c}\text { Radius } \\
(\mathrm{cm})\end{array}$} & \multirow{2}{*}{$\begin{array}{c}\text { Volume } \\
\left(\mathrm{m}^{3}\right)\end{array}$} & \multicolumn{2}{|c|}{$\begin{array}{l}\text { Dose Conversion Factor } \\
{\left[(\mathrm{Sv} / \mathrm{s}) /\left(\mathrm{Bq} / \mathrm{m}^{3}\right) \times 10^{-18}\right]}\end{array}$} & \multirow{2}{*}{$\begin{array}{c}\text { Ratio } \\
\text { FGR/MCNP }\end{array}$} \\
\hline & & & & MCNP & FGR-12 & \\
\hline Co-60 & $\begin{array}{c}1.0 \\
5.0 \\
1.5 \times 10^{1} \\
5.0 \times 10^{1} \\
1.0 \times 10^{2}\end{array}$ & $\begin{array}{l}1.0 \times 10^{4} \\
1.0 \times 10^{4} \\
1.0 \times 10^{4} \\
1.0 \times 10^{4} \\
1.0 \times 10^{4}\end{array}$ & $\begin{array}{l}3.14 \times 10^{2} \\
1.57 \times 10^{3} \\
4.71 \times 10^{3} \\
1.57 \times 10^{4} \\
3.14 \times 10^{4}\end{array}$ & $\begin{array}{l}1.42 \times 10^{1} \\
4.43 \times 10^{1} \\
7.25 \times 10^{1} \\
8.95 \times 10^{1} \\
8.73 \times 10^{1}\end{array}$ & $\begin{array}{l}1.52 \times 10^{1} \\
4.45 \times 10^{1} \\
7.25 \times 10^{1} \\
8.68 \times 10^{1} \\
8.68 \times 10^{1}\end{array}$ & $\begin{array}{c}1.07 \\
1.00 \\
1.00 \\
9.70 \times 10^{-1} \\
9.94 \times 10^{-1}\end{array}$ \\
\hline Mn-54 & $\begin{array}{c}1.0 \\
5.0 \\
1.5 \times 10^{1} \\
5.0 \times 10^{1} \\
1.0 \times 10^{2}\end{array}$ & $\begin{array}{l}1.0 \times 10^{4} \\
1.0 \times 10^{4} \\
1.0 \times 10^{4} \\
1.0 \times 10^{4} \\
1.0 \times 10^{4}\end{array}$ & $\begin{array}{l}3.14 \times 10^{2} \\
1.57 \times 10^{3} \\
4.71 \times 10^{3} \\
1.57 \times 10^{4} \\
3.14 \times 10^{4}\end{array}$ & $\begin{array}{c}4.96 \\
1.51 \times 10^{1} \\
2.38 \times 10^{1} \\
2.97 \times 10^{1} \\
2.77 \times 10^{1}\end{array}$ & $\begin{array}{c}5.21 \\
1.51 \times 10^{1} \\
2.40 \times 10^{1} \\
2.76 \times 10^{1} \\
2.76 \times 10^{1}\end{array}$ & $\begin{array}{c}1.05 \\
1.00 \\
1.01 \\
9.29 \times 10^{-1} \\
9.96 \times 10^{-1}\end{array}$ \\
\hline $\mathrm{Ra}-226$ & $\begin{array}{c}1.0 \\
5.0 \\
1.5 \times 10^{1} \\
5.0 \times 10^{1}\end{array}$ & $\begin{array}{l}1.0 \times 10^{4} \\
1.0 \times 10^{4} \\
1.0 \times 10^{4} \\
1.0 \times 10^{4}\end{array}$ & $\begin{array}{l}3.14 \times 10^{2} \\
1.57 \times 10^{3} \\
4.71 \times 10^{3} \\
1.57 \times 10^{4}\end{array}$ & $\begin{array}{l}4.13 \times 10^{-2} \\
1.20 \times 10^{-1} \\
1.71 \times 10^{-1} \\
1.86 \times 10^{-1}\end{array}$ & $\begin{array}{l}4.15 \times 10^{-2} \\
1.16 \times 10^{-1} \\
1.65 \times 10^{-1} \\
1.70 \times 10^{-1}\end{array}$ & $\begin{array}{c}1.00 \\
9.67 \times 10^{-1} \\
9.65 \times 10^{-1} \\
9.14 \times 10^{-1}\end{array}$ \\
\hline U-238 & $\begin{array}{c}1.0 \\
5.0 \\
1.5 \times 10^{1}\end{array}$ & $\begin{array}{l}1.0 \times 10^{4} \\
1.0 \times 10^{4} \\
1.0 \times 10^{4}\end{array}$ & $\begin{array}{l}3.14 \times 10^{2} \\
1.57 \times 10^{3} \\
4.71 \times 10^{3}\end{array}$ & $\begin{array}{l}4.03 \times 10^{-4} \\
5.71 \times 10^{-4} \\
5.76 \times 10^{-4}\end{array}$ & $\begin{array}{l}4.42 \times 10^{-4} \\
5.45 \times 10^{-4} \\
5.52 \times 10^{-4}\end{array}$ & $\begin{array}{c}1.10 \\
9.54 \times 10^{-1} \\
9.58 \times 10^{-1}\end{array}$ \\
\hline Al-26 & $\begin{array}{c}1.0 \\
5.0 \\
1.5 \times 10^{1} \\
5.0 \times 10^{1} \\
1.0 \times 10^{2}\end{array}$ & $\begin{array}{l}1.0 \times 10^{4} \\
1.0 \times 10^{4} \\
1.0 \times 10^{4} \\
1.0 \times 10^{4} \\
1.0 \times 10^{4}\end{array}$ & $\begin{array}{l}3.14 \times 10^{2} \\
1.57 \times 10^{3} \\
4.71 \times 10^{3} \\
1.57 \times 10^{4} \\
3.14 \times 10^{4}\end{array}$ & $\begin{array}{l}1.54 \times 10^{1} \\
4.77 \times 10^{1} \\
7.96 \times 10^{1} \\
1.00 \times 10^{2} \\
9.04 \times 10^{1}\end{array}$ & $\begin{array}{l}1.62 \times 10^{1} \\
4.74 \times 10^{1} \\
7.73 \times 10^{1} \\
9.32 \times 10^{1} \\
9.32 \times 10^{1}\end{array}$ & $\begin{array}{c}1.05 \\
9.94 \times 10^{-1} \\
9.71 \times 10^{-1} \\
9.32 \times 10^{-1} \\
1.03\end{array}$ \\
\hline
\end{tabular}

TABLE A.6 Surface Dose Comparison of MCNP and FGR-12 Values

\begin{tabular}{lccccc}
\hline & \multicolumn{5}{c}{ Surface Dose $\left[(\mathrm{Sv} / \mathrm{s}) /\left(\mathrm{Bq} / \mathrm{m}^{2}\right) \times 10^{-18}\right]$} \\
\cline { 2 - 6 } & Co-60 & Mn-54 & Ra-226 & U-238 & Al-26 \\
\hline FGR-12 & & & & & \\
MCNP & $2.35 \times 10^{3}$ & $8.10 \times 10^{2}$ & 6.44 & $5.15 \times 10^{-1}$ & $2.49 \times 10^{3}$ \\
Ratio (FGR/MCNP) & $2.05 \times 10^{3}$ & $7.25 \times 10^{2}$ & 6.16 & $4.31 \times 10^{-1}$ & $2.21 \times 10^{3}$ \\
& 1.15 & 1.12 & 1.05 & 1.19 & 1.13 \\
\hline
\end{tabular}




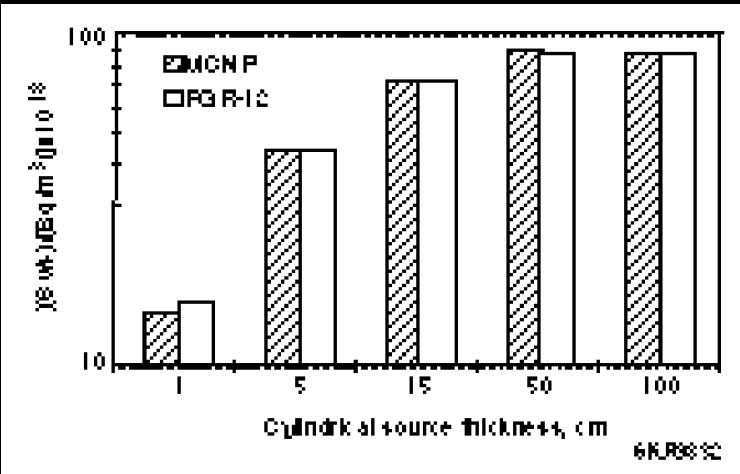

(a) $\mathbf{C o - 6 0}$

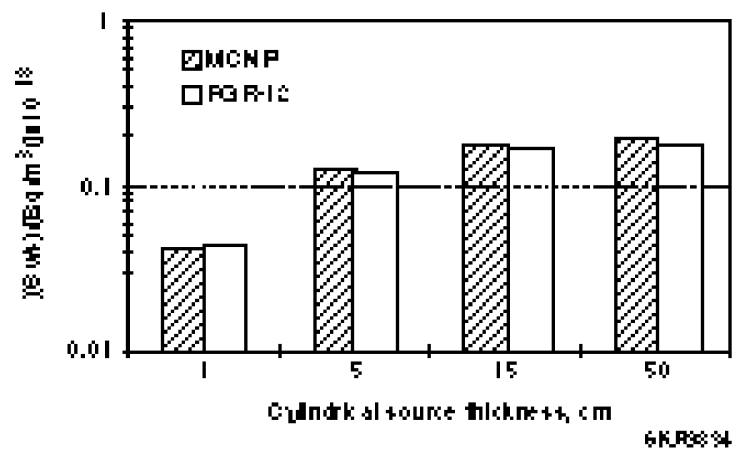

(c) Ra-226

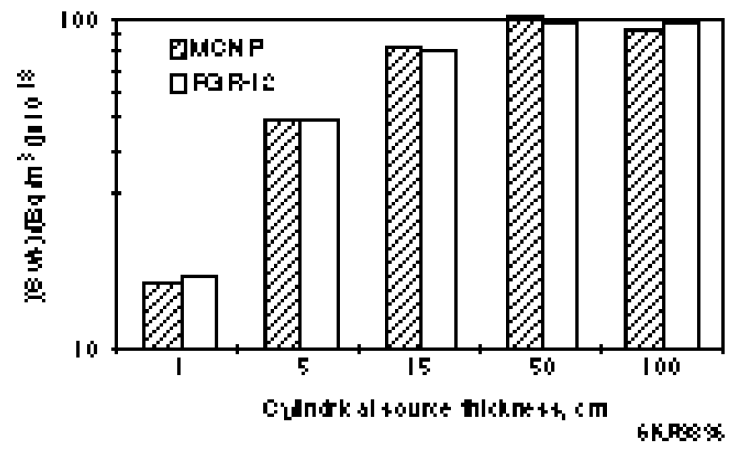

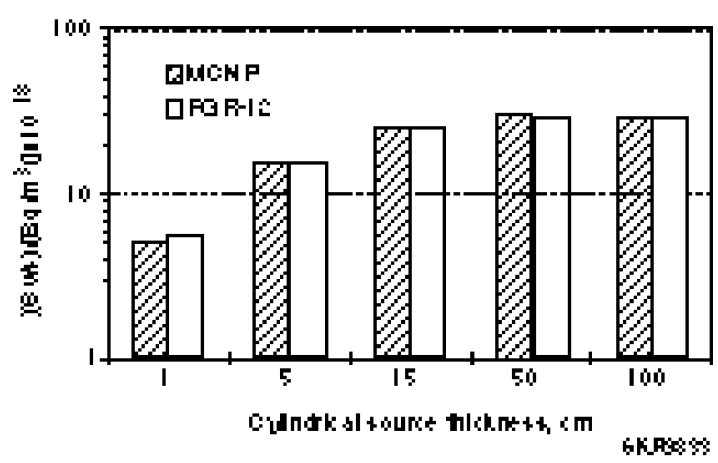

(b) Mn-54

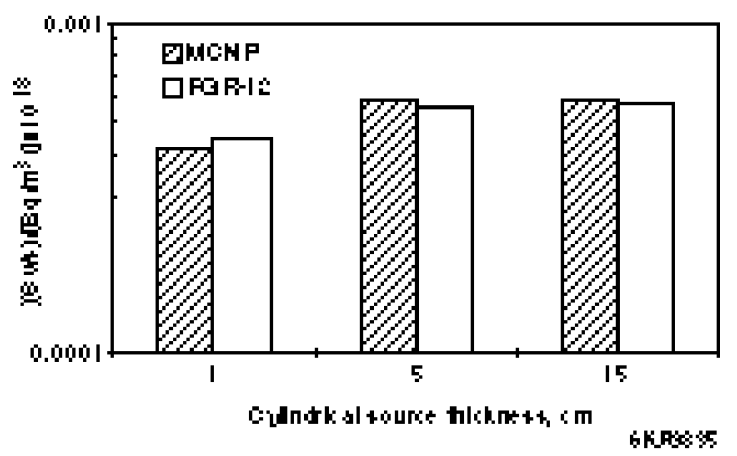

(d) $\mathbf{U}-238$

(e) Al-26

FIGURE A.1 Comparison of MCNP and FGR-12 Dose Values for Various Radionuclides 


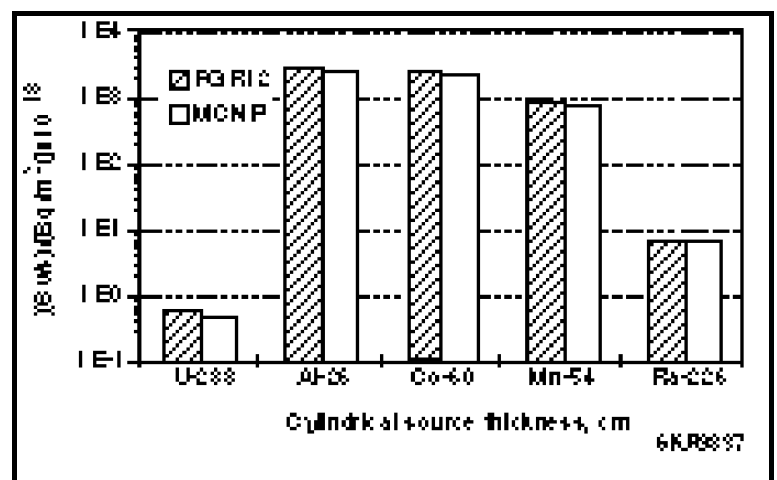

FIGURE A.2 Comparison of FGR-12 and MCNP Dose Values for Surface Sources

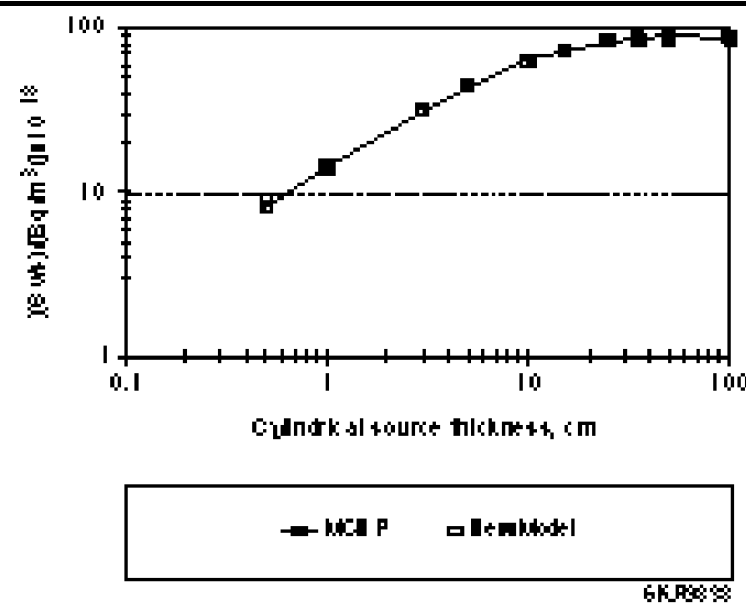

(a) Co-60

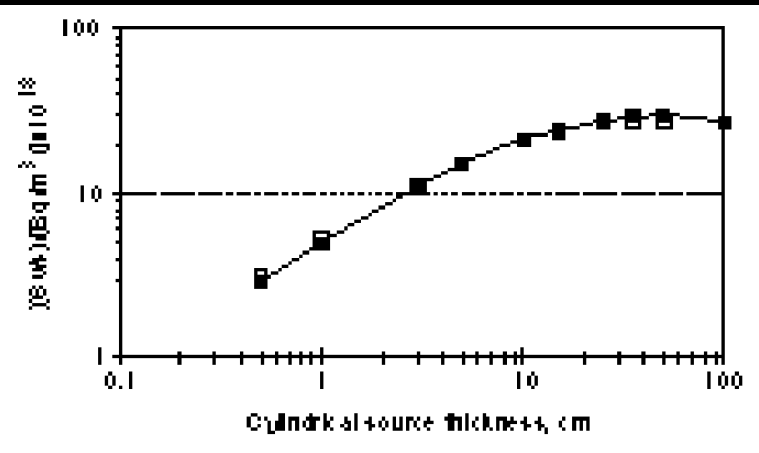

- nt] P ㅁotatel

(b) Mn-54

FIGURE A.3 Dose Comparison between MCNP and New RESRAD Model as a Function of Source Depth for Co-60 and Mn-54 
TABLE A.7 Comparison of Dose Estimation between MCNP and the New RESRAD Model for Co-60 and Mn-54 Cylindrical Sources of Effectively Infinite Radius at Different Source Depths

\begin{tabular}{|c|c|c|c|c|}
\hline \multirow[b]{2}{*}{ Isotope } & \multirow{2}{*}{$\begin{array}{c}\text { Source } \\
\text { Depth }(\mathrm{cm})\end{array}$} & \multicolumn{2}{|c|}{$\begin{array}{c}\text { Dose Estimation } \\
{\left[(\mathrm{Sv} / \mathrm{s}) /\left(\mathrm{Bq} / \mathrm{m}^{3}\right) \times 10^{-18}\right]}\end{array}$} & \multirow{2}{*}{$\begin{array}{c}\text { Ratio New } \\
\text { Model/MCNP }\end{array}$} \\
\hline & & MCNP & New Model & \\
\hline \multirow[t]{10}{*}{ Co-60 } & 0.5 & 8.17 & 9.09 & 1.11 \\
\hline & 1.0 & $1.42 \times 10^{1}$ & $1.52 \times 10^{1}$ & 1.07 \\
\hline & 3.0 & $3.18 \times 10^{1}$ & $3.17 \times 10^{1}$ & 1.00 \\
\hline & 5.0 & $4.43 \times 10^{1}$ & $4.39 \times 10^{1}$ & $9.91 \times 10^{-1}$ \\
\hline & $1.0 \times 10^{1}$ & $6.27 \times 10^{1}$ & $6.39 \times 10^{1}$ & 1.02 \\
\hline & $1.5 \times 10^{1}$ & $7.25 \times 10^{1}$ & $7.46 \times 10^{1}$ & 1.03 \\
\hline & $2.5 \times 10^{1}$ & $8.35 \times 10^{1}$ & $8.33 \times 10^{1}$ & 1.00 \\
\hline & $3.5 \times 10^{1}$ & $8.79 \times 10^{1}$ & $8.58 \times 10^{1}$ & $9.76 \times 10^{-1}$ \\
\hline & $5.0 \times 10^{1}$ & $8.95 \times 10^{1}$ & $8.66 \times 10^{1}$ & $9.68 \times 10^{-1}$ \\
\hline & $1.0 \times 10^{2}$ & $8.73 \times 10^{1}$ & $8.68 \times 10^{1}$ & $9.94 \times 10^{-1}$ \\
\hline \multirow[t]{10}{*}{ Mn-54 } & 0.5 & 2.84 & 3.17 & 1.12 \\
\hline & 1.0 & 4.96 & 5.33 & 1.07 \\
\hline & 3.0 & $1.09 \times 10^{1}$ & $1.10 \times 10^{1}$ & 1.01 \\
\hline & 5.0 & $1.51 \times 10^{1}$ & $1.51 \times 10^{1}$ & 1.00 \\
\hline & $1.0 \times 10^{1}$ & $2.11 \times 10^{1}$ & $2.14 \times 10^{1}$ & 1.01 \\
\hline & $1.5 \times 10^{1}$ & $2.38 \times 10^{1}$ & $2.45 \times 10^{1}$ & 1.03 \\
\hline & $2.5 \times 10^{1}$ & $2.78 \times 10^{1}$ & $2.68 \times 10^{1}$ & $9.64 \times 10^{-1}$ \\
\hline & $3.5 \times 10^{1}$ & $2.91 \times 10^{1}$ & $2.74 \times 10^{1}$ & $9.42 \times 10^{-1}$ \\
\hline & $5.0 \times 10^{1}$ & $2.97 \times 10^{1}$ & $2.76 \times 10^{1}$ & $9.96 \times 10^{-1}$ \\
\hline & $1.0 \times 10^{2}$ & $2.77 \times 10^{1}$ & $2.76 \times 10^{1}$ & $9.94 \times 10^{-1}$ \\
\hline
\end{tabular}


TABLE A.8 Comparison of Dose (mrem/yr) Calculations for MCNP and the New RESRAD Model Using the Cover-and-Depth Factor for Co-60 and Mn-54

\begin{tabular}{|c|c|c|c|c|c|c|c|c|}
\hline \multirow{2}{*}{$\begin{array}{c}\text { Cover } \\
\text { Thickness } \\
(\mathrm{cm})\end{array}$} & \multicolumn{2}{|c|}{ Source $=1 \mathrm{~cm}$} & \multicolumn{2}{|c|}{ Source $=5 \mathrm{~cm}$} & \multicolumn{2}{|c|}{ Source $=15 \mathrm{~cm}$} & \multicolumn{2}{|c|}{ Source $=50 \mathrm{~cm}$} \\
\hline & MCNP & $\begin{array}{c}\text { New } \\
\text { Model }\end{array}$ & MCNP & $\begin{array}{c}\text { New } \\
\text { Model }\end{array}$ & MCNP & $\begin{array}{c}\text { New } \\
\text { Model }\end{array}$ & MCNP & $\begin{array}{l}\text { New } \\
\text { Model }\end{array}$ \\
\hline \multicolumn{9}{|l|}{ Co-60 } \\
\hline 0.0 & 2.65 & 2.84 & 8.28 & 8.20 & $1.35 \times 10^{1}$ & $1.39 \times 10^{1}$ & $1.67 \times 10^{1}$ & $1.62 \times 10^{1}$ \\
\hline 0.5 & 2.00 & 2.04 & 7.15 & 7.00 & $1.23 \times 10^{1}$ & $1.24 \times 10^{1}$ & $1.48 \times 10^{1}$ & $1.45 \times 10^{1}$ \\
\hline 2.0 & 1.41 & 1.39 & 5.4 & 5.44 & 10.1 & 9.90 & $1.30 \times 10^{1}$ & $1.16 \times 10^{1}$ \\
\hline 5.0 & $8.9 \times 10^{-1}$ & $9.43 \times 10^{-1}$ & 3.7 & 3.73 & 7.02 & 6.79 & 9.4 & 7.99 \\
\hline $1.0 \times 10^{1}$ & $5.0 \times 10^{-1}$ & $5.04 \times 10^{-1}$ & 2.05 & 1.99 & 4.26 & 3.63 & 5.7 & 4.28 \\
\hline $2.5 \times 10^{1}$ & $1.1 \times 10^{-1}$ & $7.70 \times 10^{-2}$ & $4.6 \times 10^{-1}$ & $3.05 \times 10^{-1}$ & $9.1 \times 10^{-1}$ & $5.54 \times 10^{-1}$ & 1.3 & $6.51 \times 10^{-1}$ \\
\hline \multicolumn{9}{|l|}{ Mn-54 } \\
\hline 0.0 & $9.27 \times 10^{-1}$ & $9.96 \times 10^{-1}$ & 2.82 & 2.82 & 4.45 & 4.59 & 5.64 & 5.16 \\
\hline 0.5 & $7.27 \times 10^{-1}$ & $7.22 \times 10^{-1}$ & 2.43 & 2.39 & 4.05 & 4.04 & 4.87 & 4.56 \\
\hline 2.0 & $4.82 \times 10^{-1}$ & $4.76 \times 10^{-1}$ & 1.81 & 1.81 & 3.26 & 3.14 & 3.87 & 3.57 \\
\hline 5.0 & $3.03 \times 10^{-1}$ & $3.07 \times 10^{-1}$ & 1.18 & 1.18 & 2.23 & 2.05 & 2.62 & 2.33 \\
\hline $1.0 \times 10^{1}$ & $1.61 \times 10^{-1}$ & $1.52 \times 10^{-1}$ & $6.33 \times 10^{-1}$ & $5.83 \times 10^{-1}$ & 1.20 & 1.02 & 1.46 & 1.16 \\
\hline $2.5 \times 10^{1}$ & $2.8 \times 10^{-2}$ & $1.84 \times 10^{-2}$ & $1.12 \times 10^{-1}$ & $7.07 \times 10^{-2}$ & $1.98 \times 10^{-1}$ & $1.23 \times 10^{-1}$ & $2.43 \times 10^{-1}$ & $1.40 \times 10^{-1}$ \\
\hline
\end{tabular}




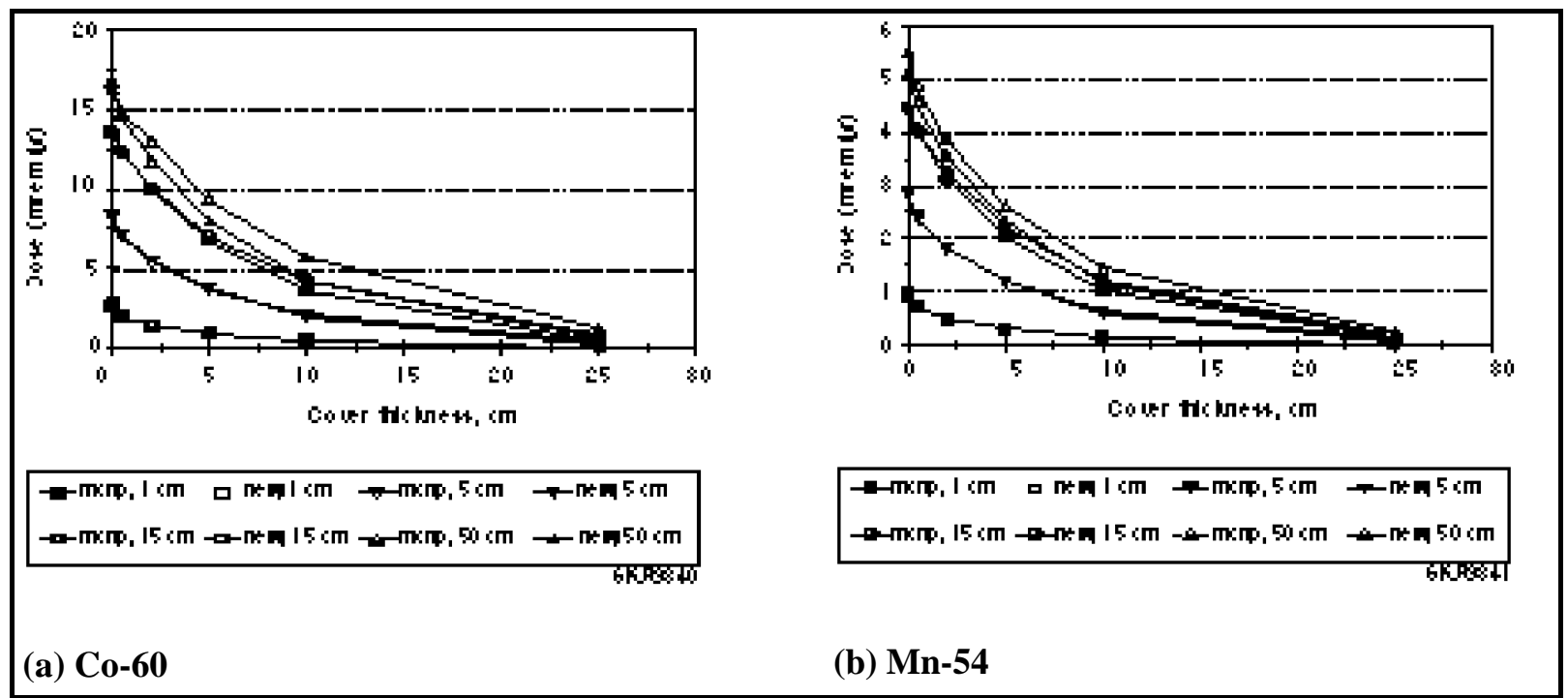

FIGURE A.4 Dose Comparison between MCNP and New RESRAD Model for Co-60 and Mn-54 as a Function of Cover Thickness for Different Source Depths

TABLE A.9 Ratio of New RESRAD Model/MCNP Dose Calculations as a Function of Cover Thickness for Different Source Depths for Co-60 and Mn-54

\begin{tabular}{|c|c|c|c|c|}
\hline \multirow{2}{*}{$\begin{array}{l}\text { Cover } \\
\text { Thickness } \\
(\mathrm{cm})\end{array}$} & \multicolumn{4}{|c|}{ Ratio (New Model/MCNP) by Source Depth } \\
\hline & $1 \mathrm{~cm}$ & $5 \mathrm{~cm}$ & $15 \mathrm{~cm}$ & $50 \mathrm{~cm}$ \\
\hline \multicolumn{5}{|l|}{ Co-60 } \\
\hline 0.0 & 1.07 & $9.90 \times 10^{-1}$ & 1.03 & $9.70 \times 10^{-1}$ \\
\hline 0.5 & 1.02 & $9.79 \times 10^{-1}$ & 1.01 & $9.80 \times 10^{-1}$ \\
\hline 2.0 & $9.86 \times 10^{-1}$ & 1.01 & $9.80 \times 10^{-1}$ & $8.92 \times 10^{-1}$ \\
\hline 5.0 & 1.06 & 1.01 & $9.67 \times 10^{-1}$ & $8.50 \times 10^{-1}$ \\
\hline $1.0 \times 10^{1}$ & 1.01 & $9.71 \times 10^{-1}$ & $8.52 \times 10^{-1}$ & $7.51 \times 10^{-1}$ \\
\hline $2.5 \times 10^{1}$ & $7.00 \times 10^{-1}$ & $6.63 \times 10^{-1}$ & $6.09 \times 10^{-1}$ & $5.01 \times 10^{-1}$ \\
\hline \multicolumn{5}{|l|}{ Mn-54 } \\
\hline 0.0 & 1.07 & 1.00 & 1.03 & $9.15 \times 10^{-1}$ \\
\hline 0.5 & $9.93 \times 10^{-1}$ & $9.84 \times 10^{-1}$ & $9.98 \times 10^{-1}$ & $9.36 \times 10^{-1}$ \\
\hline 2.0 & $9.88 \times 10^{-1}$ & 1.00 & $9.63 \times 10^{-1}$ & $9.22 \times 10^{-1}$ \\
\hline 5.0 & 1.01 & 1.00 & $9.19 \times 10^{-1}$ & $8.89 \times 10^{-1}$ \\
\hline $1.0 \times 10^{1}$ & $9.44 \times 10^{-1}$ & $9.21 \times 10^{-1}$ & $8.50 \times 10^{-1}$ & $7.95 \times 10^{-1}$ \\
\hline $2.5 \times 10^{1}$ & $6.57 \times 10^{-1}$ & $6.31 \times 10^{-1}$ & $6.21 \times 10^{-1}$ & $5.76 \times 10^{-1}$ \\
\hline
\end{tabular}




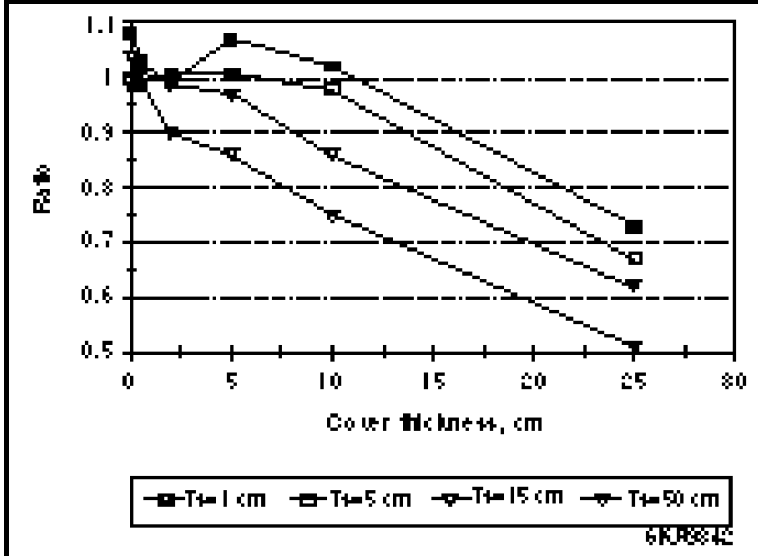

(a) $\mathrm{Co-60}$

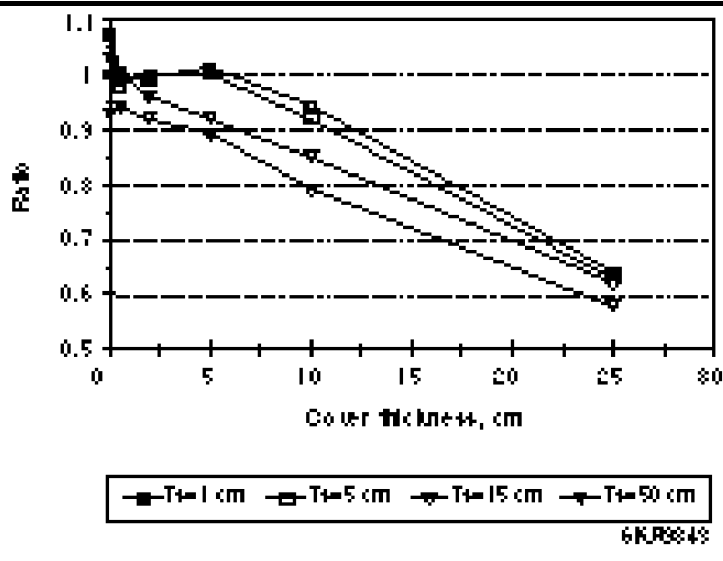

(b) Mn-54

FIGURE A.5 Ratio of Cover-and-Depth Factor for Co-60 and Mn-54 at Different Source Depths

The above results could also arise from the use of different exposure models (rotational exposure [MCNP] and the actual field [FGR-12]) in effective dose equivalent calculations. An indication of support for this interpretation comes from FGR-12 (Figure II.16 in Eckerman and Ryman 1993), where the rotational exposures were 10-35\% higher than actual fields, with the larger difference at greater source thicknesses.

\section{A.4 REFERENCES FOR APPENDIX}

Briesmeister, J.F. (editor), 1993, MCNP - A General Monte Carlo N-Particle Transport Code, Version 4A, LA-12625, Los Alamos National Laboratory, Los Alamos, N.M.

Eckerman, K.F., and J.C. Ryman, 1993, External Exposure to Radionuclides in Air, Water, and Soil, Exposure to Dose Coefficients for General Application, Based on the 1987 Federal Radiation Protection Guidance, EPA 402-R-93-081, Federal Guidance Report No. 12, prepared by Oak Ridge National Laboratory, Oak Ridge, Tenn., for U.S. Environmental Protection Agency, Office of Radiation and Indoor Air, Washington, D.C.

ICRP: See International Commission on Radiological Protection

International Commission on Radiological Protection, 1983, Radionuclide Transformations: Energy and Intensity of Emissions, ICRP Publication 38, Annals of the ICRP, Vols. 11-13, Pergamon Press, New York, N.Y. 
International Commission on Radiological Protection, 1987, Data for Use in Protection against External Radiation, ICRP Publication 51, Annals of the ICRP, Vol. 17, No. 2/3, Pergamon Press, New York, N.Y. 